\title{
Zeros de polinômios \\ e propriedades polinomiais em espaços de Banach
}

\section{Neusa Nogas Tocha}

\author{
TESE APRESENTADA \\ $\mathrm{AO}$ \\ INSTituTO DE MATEMÁTICA E ESTATÍSTICA \\ DA \\ UNIVERSIDADE DE SÃO PAULO \\ PARA \\ OBTENÇÃO DO TÍTULO DE DOUTORA \\ EM \\ CIÊNCIAS \\ Área de concentração: Matemática \\ Orientadora: Profa. Dra. Mary Lilian Lourenço
}

Durante a elaboração deste trabalho a autora recebeu auxílio financeiro do $C N P q$

São Paulo, abril de 2006 



\title{
Zeros de polinômios \\ e propriedades polinomiais em espaços de Banach
}

\author{
Neusa Nogas Tocha \\ Orientadora: Profa. Dra. Mary Lilian Lourenço
}

Tese apresentada ao Instituto de Matemática e Estatística da Universidade de São Paulo para a obtenção do título de

Doutora em Ciências área de concentração: Matemática

Durante a elaboração deste trabalho a autora recebeu auxílio financeiro do CNPq

São Paulo, abril de 2006 



\title{
Zeros de polinômios \\ e propriedades polinomiais em espaços de Banach
}

\author{
Este exemplar corresponde à redação final \\ da tese devidamente corrigida e \\ defendida por Neusa Nogas Tocha \\ e aprovada pela comissão julgadora.
}

São Paulo, 06 de abril de 2006.

Banca examinadora:

- Profa. Dra. Mary Lilian Lourenço (orientadora) - IME-USP

- Prof. Dr. Humberto Daniel Carrión Villarreal - IME-USP

- Prof. Dr. Jorge Túlio Mujica Ascui - IMECC-UNICAMP

- Prof. Dr. Geraldo Márcio de Azevedo Botelho - UFU

- Prof. Dr. Raymundo Luiz Alencar - ITA 

Dedico este trabalho

à Nossa Senhora Aparecida por proteger a mim e a minha família 



\section{Agradecimentos}

Agradeço a Deus pela vida e coragem para nela enfrentar os muitos obstáculos que transpus para chegar até aqui.

Agradedeço à minha orientadora pela sua orientação, pela extrema dedicação, pelo incentivo e sugestões que tornaram possível a realização deste trabalho.

Agradeço a amizade de todos os colegas do IME-USP, em especial, à Andréia C., ao Caio, à Fernanda Ester C., à Hilde, à Lourdes, ao Márcio F. e à Patrícia Hess.

Agradeço aos professores do IME-USP que, diretamente ou indiretamente, contribuíram na minha formação acadêmica.

Agradeço ao IME e à USP pela oportunidade de fazer a minha pós-graduação aqui e pelas condições que me ofertaram (biblioteca, laboratório de informática e auxílio financeiro para diversos eventos).

Agradeço aos funcionários do IME pelo bom atendimento, em particular, ao pessoal da biblioteca (BIME) e da CPG.

Agradeço ao CNPq pelo apoio financeiro recebido durante o programa de Doutorado.

Agradeço ao meu esposo Maurício pelo seu apoio e sua compreensão, aos meus pais pelo incentivo nos estudos e aos meus irmãos Nilson, Estefano e Gabriel pelas suas atitudes, pequenas mas grandiosas, que muito me ajudaram.

Gostaria de deixar aqui registrado um agradecimento muito especial as seguintes pessoas: À Simone K. por ter cedido gentilmente um espaço no seu AP do CRUSP durante o $1^{\circ}$ semestre de 1999. Aos professores da UFPR, Carlos Henrique, Edson Álvares, José Carlos Cifuentes e José João Rosseto pelo incentivo à carreira acadêmica. À profa. Lirani, professora de matemática no ensino médio, pelo incentivo e apoio durante os anos que fui sua aluna.

Agradeço a todos vocês pelo companheirismo durante todos estes anos.

( $\star$ ) Muito obrigado $(\star)$ 


\section{Sumário}

Notação $\quad 5$

$\begin{array}{ll}\text { Introdução } & 9\end{array}$

1 Conceitos Preliminares $\quad 11$

1.1 Espaços de Banach Especiais . . . . . . . . . . . . . . . . . . . 14

1.1.1 Espaço de Stegall . . . . . . . . . . . . . . . . . . . . . . . 14

1.1.2 Espaço de Baernstein . . . . . . . . . . . . . . . 16

1.1.3 Espaço de Lorentz . . . . . . . . . . . . . . . . . . . . . 17

2 Espaços que possuem as propriedades (P) e (RP) 19

2.1 Funções com a propriedade ACL . . . . . . . . . . . . . . . . . . 20

2.2 A condição $(\star \star)$ e a propriedade $(\mathbf{P}) \ldots \ldots$. . . . . . . . . . . . 26

2.3 A propriedade $(\mathbf{R P}) \ldots \ldots \ldots \ldots \ldots$

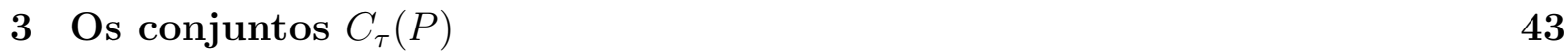

3.1 O conjunto $C(P) \ldots \ldots \ldots \ldots \ldots$

3.2 O conjunto $C_{\omega^{\star}}(P) \ldots \ldots \ldots \ldots \ldots \ldots$ 
$4 \quad$ Zeros de polinômios $\quad 63$

4.1 Introdução . . . . . . . . . . . . . . . . . . . . . . 63

4.2 Limitação para $d \geq 3 \ldots \ldots \ldots$. . . . . . . . . . . . . . . . . . . . . .

$\begin{array}{ll}\text { Referências Bibliográficas } & 75\end{array}$ 


\section{Resumo}

Neste trabalho temos por objetivo apresentar alguns resultados relacionados aos temas abordados por Aron, Choi e Llavona (1995), Aron e Dimant (2002) e Aron e Rueda (1997). Primeiramente, vamos estudar as propriedades polinomiais $(\mathbf{P})$ e $(\mathbf{R P})$ para os espaços de Banach e a propriedade ACL para as funções definidas entre as bolas unitárias fechadas do espaço. Vamos apresentar novos exemplos de espaços de Banach que possuem a propriedade $(\mathbf{P})$ onde é possível exibir funções que satisfazem a propriedade ACL. Vamos ainda estudar o conjunto de continuidade seqüencial fraca de um polinômio $N$-homogêneo contínuo com valores vetoriais. Apresentamos as suas propriedades básicas e algumas conexões com o caso dos polinômios escalares. No espaço dual faremos uma breve análise dos polinômios com certo tipo de continuidade com relação à topologia fraca-estrela. Numa outra direção, estudamos os zeros de polinômios $N$-homogêneos em várias variáveis complexas, mais especificamente, dados $n, N \in \mathbb{N}$ existe $m \in \mathbb{N}$ tal que para cada polinômio $N$-homogêneo $P: \mathbb{C}^{m} \longrightarrow \mathbb{C}$ existe um subespaço vetorial $X_{P} \subset P^{-1}(0)$ de dimensão $n$. Aqui, o principal objetivo é melhorar as limitações para $m$ encontradas por Aron e Rueda (1997) como também generalizar os seus resultados. 


\section{Abstract}

Our purpose here is to study some results regarding the articles of Aron, Choi and Llavona (1995), Aron and Dimant (2002) and Aron and Rueda (1997). Firstly, we study properties (P) and (RP) for the Banach spaces and the ACL property for the functions defined between the closed unit balls. We give new examples of Banach spaces which have (P) property and some functions defined in those spaces satisfying the ACL property. We also study the set of weak sequential continuity of a vector-valued continuous $\mathrm{N}$ homogeneous polynomial. In the dual space we study the $N$-homogeneous polynomials which are weak-star continuous on bounded sets. Finally, we study the zeros of complex $N$-homogeneous polynomials. This means, given positive integers $n$ and $N$, there is a positive integer $m$ such that an $N$-homogeneous polynomial $P: \mathbb{C}^{m} \longrightarrow \mathbb{C}$ has an $n$ dimensional subspace contained in its zero set. We discuss the problem of finding a good bound on $m$ as a function of $n$ and $N$. We improve the results given by Aron and Rueda (1997) as also generalize their results. 


\section{Notação}

No que segue vamos apresentar as notações necessárias que serão utilizadas no decorrer deste trabalho.

$\begin{array}{ll}\mathbb{N} & \text { o conjunto dos números naturais } \\ \mathbb{N}_{0} & \mathbb{N} \cup\{0\} \\ \Gamma & \{\gamma \subset \mathbb{N},|\gamma| \leq \min \gamma\} \\ \mathbb{R} & \text { o corpo dos números reais } \\ \mathbb{C} & \text { o corpo dos números complexos } \\ \mathbb{K} & \text { o corpo } \mathbb{R} \text { ou } \mathbb{C} \\ E, F & \text { espaços de Banach sobre } \mathbb{K} \\ B_{E} & \text { a bola unitária fechada de } E: x \in E \text { com }\|x\| \leq 1 \\ S_{E} & \text { a esfera unitária de } E: x \in E \text { com }\|x\|=1 \\ E^{\prime} & \text { o espaço dual de } E: f: E \longrightarrow \mathbb{K} \text { linear e contínua } \\ {\left[\left(x_{n}\right)_{n}\right]} & \text { subespaço fechado de } E \text { gerado por }\left(x_{n}\right)_{n} \\ \|\cdot\|_{b m u\left(x_{n}\right)} & \text { norma incondicional multiplicador limitado relativa à base }\left(x_{n}\right)_{n} \\ \delta_{n j} & \text { Símbolo de Kronecher onde } \delta_{n j}=\left\{\begin{array}{l}0 \quad \text { se } \\ \end{array}\right. \\ & \end{array}$




$$
\begin{aligned}
& F \hookrightarrow E \quad E \text { contém uma cópia isomorfa de } F \\
& F \nrightarrow E \quad E \text { não contém cópia isomorfa de } F \\
& \omega, \omega^{\star} \quad \text { topologias fraca e fraca-estrela, respectivamente } \\
& x_{n} \stackrel{n \rightarrow \infty}{\longrightarrow} x \quad \text { a seqüência }\left(x_{n}\right)_{n} \text { converge para } x \text { na topologia da norma } \\
& x_{n} \stackrel{\omega}{\rightarrow} x, x_{n} \stackrel{\omega^{\star}}{\rightarrow} x \quad \text { a seqüência }\left(x_{n}\right)_{n} \text { converge para } x \text { na topologia } \omega, \omega^{\star} \text { respectivamente } \\
& \mathcal{L}(E ; F) \quad \text { espaço das aplicações lineares contínuas } T: E \longrightarrow F \\
& \mathcal{L}_{s}\left({ }^{m} E ; F\right) \quad A: E \times \stackrel{(m)}{*} \times E \longrightarrow F m \text {-lineares simétricas contínuas } \\
& \mathcal{P}\left({ }^{m} E ; F\right) \quad \text { espaço dos polinômios } m \text {-homogêneos contínuos } P: E \longrightarrow F \\
& \mathcal{P}\left({ }^{m} E\right) \quad \mathcal{P}\left({ }^{m} E ; F\right) \text { para } F=\mathbb{K} \\
& \mathcal{P}_{w u}\left({ }^{m} E\right) \quad P \in \mathcal{P}\left({ }^{m} E\right) \omega \text {-uniformemente contínuo sobre os limitados } \\
& \mathcal{P}_{\text {wsc }}\left({ }^{m} E ; F\right) \quad P \in \mathcal{P}\left({ }^{m} E ; F\right) \omega \text {-seqüencialmente contínuos em } E \\
& \mathcal{P}_{\text {wsc }(0)}\left({ }^{m} E ; F\right) \quad P \in \mathcal{P}\left({ }^{m} E ; F\right) \omega \text {-seqüencialmente contínuos na origem } \\
& \mathcal{P}_{w^{\star} s c}\left({ }^{m} E ; F\right) \quad P \in \mathcal{P}\left({ }^{m} E ; F\right) \omega^{\star} \text {-seqüencialmente contínuos em } E \\
& \left.\mathcal{P}_{w^{\star} s c(0)}{ }^{m} E ; F\right) \quad P \in \mathcal{P}\left({ }^{m} E ; F\right) \omega^{\star} \text {-seqüencialmente contínuos na origem } \\
& \mathcal{P}_{w^{\star} b}\left({ }^{m} E ; F\right) \quad P \in \mathcal{P}\left({ }^{m} E ; F\right) \omega^{\star} \text {-contínuos sobre os limitados de } E \\
& \left.\mathcal{P}_{w^{\star} b(0)}{ }^{m} E ; F\right) \quad P \in \mathcal{P}\left({ }^{m} E ; F\right) \omega^{\star} \text {-contínuos } \forall W \subset E \text { limitado com } 0 \in W \\
& \mathcal{P}_{f}\left({ }^{m} E ; F\right) \quad P \in \mathcal{P}\left({ }^{m} E ; F\right) \text { onde } P \text { é um polinômio do tipo finito } \\
& \mathbb{P}_{f}\left({ }^{m} E ; F\right) \quad\left\{P \in \mathcal{P}\left({ }^{N} E ; F\right): P=\sum_{i=1}^{k} T_{i}^{N}, T_{i} \in \mathcal{L}(E ; F)\right\} \\
& \mathcal{H}(E) \quad \text { espaço das funções holomorfas } f: E \longrightarrow \mathbb{K} \\
& \mathcal{H}_{b}(E) \quad f \in \mathcal{H}(E): f \text { é do tipo limitada } \\
& C(P) \quad x \in E: P \text { é } \omega \text {-seqüencialmente contínuo em } x \\
& C_{w^{\star}}(P) \quad x \in E: P \text { é } \omega^{\star} \text {-contínuo } \forall W \subset E \text { limitado com } x \in W \\
& C_{\star}(P) \quad x \in E: P \text { é } \omega^{\star} \text {-seqüencialmente contínuo em } x
\end{aligned}
$$




$$
\begin{array}{ll}
\left(\bigoplus_{n=1}^{\infty} l_{2}^{(n)}\right)_{1},\left(\bigoplus_{n=1}^{\infty} l_{2}^{(n)}\right)_{\infty},\left(\bigoplus_{n=1}^{\infty} l_{2}^{(n)}\right)_{0} & \text { espaço de Stegall, seu dual e predual respec- } \\
& \text { tivamente, ver [38] } \\
\mathbb{B}_{\mathbf{p}}, 1<p<\infty & \text { espaço de Baernstein ver }[10] \\
d(w ; p), d_{*}(w ; p), 1 \leq p<\infty & \text { espaço de Lorentz e seu predual respectiva- } \\
& \text { mente }[20,21] \\
d^{*}(w ; 1) & \text { dual de } d(w ; 1) \\
\mathcal{S} & \text { espaço de Schreier ver }[18] \text { construção } 0.2 \text { pg } 1 \\
T_{J}^{*} & \text { espaço de Tsirelson*-James [6] pg 1016-1017. }
\end{array}
$$

As propriedades topológicas envolvidas no decorrer do trabalho estarão associadas à topologia dada pela norma em questão. A menos que se mencione algo, vamos utilizar as notações descritas acima. 


\section{Introdução}

Nos últimos anos tem sido grande o interesse em estabelecer relações entre os polinômios $N$-homogêneos contínuos e as propriedades geométricas dos espaços de Banach no qual eles estão definidos. Por isso, os polinômios $N$-homogêneos contínuos têm sido objeto de pesquisa de inúmeros matemáticos. Por exemplo, Aron, Choi e Llavona [4] introduzem as propriedades polinomiais (P) e (RP) e estudam as suas relações com determinados tipos de espaços de Banach. Aron e Dimant [5] estudam o conjunto de continuidade seqüencial fraca de um polinômio $N$-homogêneo contínuo com valores escalares, estabelecendo interessantes conexões entre as propriedades topológicas e algébricas de polinômios. Boyd e Ryan [11] estudam o conjunto de fraca continuidade uniforme sobre os limitados obtendo algumas identificações para o espaço dos polinômios $N$-homogêneos $\omega$-contínuos $P: E \longrightarrow \mathbb{K}$. Seguindo em outra direção, Aron e Rueda [9] estudam a existência de subespaços nos zeros de determinados polinômios.

É dentro do contexto dos textos científicos acima citados que este trabalho se desenvolve. No que segue descreveremos os assuntos abordados em cada capítulo.

No capítulo 1, são apresentados alguns conceitos e resultados sobre as bases de Schauder, as propriedades de Dunford-Pettis e polinomialmente Schur e alguns espaços de Banach que utilizaremos no decorrer do trabalho.

Dando prosseguimento ao projeto desenvolvido durante o mestrado [39], no capítulo 2, o tema a ser abordado é o estudo das propriedades ACL, (P) e (RP). Versões preliminares dos resultados aqui expostos foram apresentados nas edições 61 e 62 do Seminário 
Brasileiro de Análise, [41, 42].

Na seção 2.1 vamos apresentar condições em um espaço de Banach $E$ e exibir funções $g$ definidas entre as bolas unitárias fechadas que não são uniformemente contínuas e satisfazem a seguinte propriedade ACL: Para qualquer polinômio $N$-homogêneo contínuo $P: E \longrightarrow \mathbb{K}, P \circ g: B_{E} \longrightarrow \mathbb{K}$ é uniformemente contínua. Na seção 2.2 vamos estudar a propriedade $(\mathbf{P})$ em determinados espaços de Banach. Esta propriedade nos auxilia a determinar espaços de Banach onde as funções $g: B_{E} \longrightarrow B_{E}$ que satisfaçam a propriedade ACL sejam uniformemente contínuas. E na seção 2.3 vamos estudar a recíproca da propriedade $(\mathbf{P})$, denominada de propriedade $(\mathbf{R P})$, em determinados espaços de Banach.

O interesse sobre o conjunto de continuidade seqüencial fraca de um polinômio $N$ homogêneo contínuo $P: E \longrightarrow \mathbb{K}$ tem como origem o fato de que nem todo polinômio é fracamente seqüencialmente contínuo em todo o domínio ou em ponto algum. Em muitos espaços de Banach os polinômios fracamente seqüencialmente contínuos com valores vetoriais têm comportamentos totalmente distintos do caso escalar. No capítulo 3 vamos estudar o conjunto de continuidade seqüencial fraca dos polinômios $N$-homogêneos contínuos $P: E \longrightarrow F$, denotado por $C(P)$. Na seção 3.1 estudaremos as suas propriedades básicas e algumas conexões com os resultados obtidos por Aron e Dimant em [5]. Apresentaremos condições necessárias tais que para cada $Q \in \mathcal{P}_{w s c}\left({ }^{M} E\right)$ exista $R \in \mathcal{P}\left({ }^{N} E ; F\right)$ que satisfaça $C(R)=Q^{-1}(0)$. Também vamos analisar o conjunto $C(P)$ para determinados $P \in \overline{\mathbb{P}_{f}\left({ }^{N} E ; F\right)}\|\cdot\|$. E na seção 3.2 vamos analisar os polinômios que são $\omega^{\star}$-contínuos sobre os limitados como também a continuidade seqüencial $\omega^{\star}$, com $E$ sendo um espaço dual.

No capítulo 4, o tema a ser abordado é o estudo dos zeros de polinômios $N$-homogêneos em várias variáveis complexas, mais especificamente, dados $n, N \in \mathbb{N}$ existe $m \in \mathbb{N}$ tal que para cada polinômio $N$-homogêneo $P: \mathbb{C}^{m} \longrightarrow \mathbb{C}$ existe um subespaço vetorial $X_{P} \subset P^{-1}(0)$ de dimensão $n$.

Para $N \geq 3$, não é conhecido um valor preciso de $m$ para qualquer $n$. Aron e Rueda em [9] discutem o problema de calcular uma melhor limitação para $m$ em função de $n$ e $N$ para $N=2,3$ e 4 . Aqui, melhoramos os resultados obtidos em [9], quando $N \geq 3$. 


\section{Capítulo 1}

\section{Conceitos Preliminares}

Neste capítulo vamos apresentar alguns conceitos e resultados que serão necessários no desenvolvimento deste trabalho.

Seja $E$ um espaço de Banach. Uma seqüência $\left(x_{n}\right)_{n} \subset E$ é uma base de Schauder para $E$ se para cada $x \in E$ é possível obter uma única seqüência de escalares $\left(\alpha_{n}\right)_{n}$ tal que $x=\sum_{n=1}^{\infty} \alpha_{n} x_{n}$. E neste caso, para cada $m \in \mathbb{N}$, definimos o $m$-ésimo funcional coordenado $x_{m}^{\prime}$ relativo à base $\left(x_{n}\right)_{n}$ como sendo a aplicação $x_{m}^{\prime}: E \longrightarrow \mathbb{K}$ dada por: $x_{m}^{\prime}\left(\sum_{n=1}^{\infty} \alpha_{n} x_{n}\right)=\alpha_{m}$ para cada $x=\sum_{n=1}^{\infty} \alpha_{n} x_{n} \in E$.

Definições 1.0.1. Seja $\left(x_{n}\right)_{n}$ uma base de Schauder para E.

Dado $x^{\prime} \in E^{\prime}$ definamos, para cada $m \in \mathbb{N},\left\|x^{\prime}\right\|_{(m)}=\left\|\left.x^{\prime}\right|_{\left[x_{n}: n>m\right]}\right\|$.

Dizemos que uma base $\left(x_{n}\right)_{n}$ é contrátil quando, para cada $x^{\prime} \in E^{\prime}, \lim _{m \rightarrow \infty}\left\|x^{\prime}\right\|_{(m)}=0$.

Dizemos que uma base $\left(x_{n}\right)_{n}$ é limitadamente completa quando para toda seqüência de escalares $\left(\alpha_{n}\right)_{n}$ com $\sup _{m}\left\|\sum_{n=1}^{m} \alpha_{n} x_{n}\right\|<\infty$ tem-se que a série $\sum_{n=1}^{\infty} \alpha_{n} x_{n}$ converge em $E$. Dizemos que uma base $\left(x_{n}\right)_{n}$ é incondicional se a expansão $x=\sum_{n=1}^{\infty} \alpha_{n} x_{n}$ é uma série incondicionalmente convergente para cada $x \in E$, ou seja, $\sum_{n=1}^{\infty} \alpha_{\sigma(n)} x_{\sigma(n)}$ é convergente para toda permutação $\sigma$ dos naturais.

Dentre os resultados sobre bases de Schauder, destacamos o seguinte: 
Teorema 1.0.2 (Megginson, [30]). Sejam $\left(x_{n}\right)_{n}$ uma base de Schauder para E e $\left(x_{n}^{\prime}\right)_{n}$ a seqüência dos funcionais coordenados associados à base $\left(x_{n}\right)_{n}$. Então:

1. $\left(x_{n}^{\prime}\right)_{n}$ é uma base de Schauder para $E^{\prime}$ se e somente se $\left(x_{n}\right)_{n}$ é contrátil (proposição $4.4 .7)$.

2. E é reflexivo se e somente se $\left(x_{n}\right)_{n}$ é contrátil e limitadamente completa (teorema $4 \cdot 4 \cdot 15)$.

Seja $\left(x_{n}\right)_{n}$ uma base incondicional para $E$. Dado $x=\sum_{n=1}^{\infty} \alpha_{n} x_{n} \in E$ temos que $\sum_{n=1}^{\infty} \beta_{n} \alpha_{n} x_{n} \in E$ para cada seqüência $\left(\beta_{n}\right)_{n} \in l_{\infty}$ (The Bounded Multiplier Test, ver por exemplo Megginson, [30] 4.2.8). No capítulo 2 vamos trabalhar com a seguinte norma:

Definição 1.0.3. Seja $\left(x_{n}\right)_{n}$ uma base incondicional para E. Definamos a norma incondicional multiplicador limitado relativa à base $\left(x_{n}\right)_{n}$ como sendo:

$$
\left\|\sum_{n=1}^{\infty} \alpha_{n} x_{n}\right\|_{b m u\left(x_{n}\right)}=\sup \left\{\left\|\sum_{n=1}^{\infty} \beta_{n} \alpha_{n} x_{n}\right\|:\left(\beta_{n}\right)_{n} \in S_{l_{\infty}}\right\}<\infty
$$

para cada $x=\sum_{n=1}^{\infty} \alpha_{n} x_{n} \in E$.

O espaço $E$ munido da norma $\|\cdot\|_{b m u\left(x_{n}\right)}$ é um espaço de Banach, a norma $\|\cdot\|_{b m u\left(x_{n}\right)}$ é equivalente à norma original de $E$ e satisfaz $\|x\| \leq\|x\|_{b m u\left(x_{n}\right)} \leq\left\|I d_{E}^{-1}\right\|\|x\|$ para cada $x \in E$, onde $I d_{E}:\left(E,\|\cdot\|_{b m u\left(x_{n}\right)}\right) \longrightarrow(E,\|\cdot\|)$ (ver por exemplo Megginson, [30] teorema 4.2.16). De imediato, segue que $\left\|\sum_{n=1}^{\infty} \beta_{n} \alpha_{n} x_{n}\right\|_{b m u\left(x_{n}\right)} \leq\left\|\left(\beta_{n}\right)_{n}\right\|_{\infty}\left\|\sum_{n=1}^{\infty} \alpha_{n} x_{n}\right\|_{b m u\left(x_{n}\right)}$ para todos $\sum_{n=1}^{\infty} \alpha_{n} x_{n} \in E$ e $\left(\beta_{n}\right)_{n} \in l_{\infty}$ (ver Megginson, [30] proposição 4.2.17).

Dado um espaço de Banach $E$ com base incondicional $\left(x_{n}\right)_{n}$, vamos denotar por $B_{b m u\left(x_{n}\right)(E)}:=\left\{x \in E:\|x\|_{b m u\left(x_{n}\right)} \leq 1\right\}$. De imediato temos que $B_{b m u\left(x_{n}\right)(E)} \subset B_{E}$.

Nos espaços de seqüências, se a seqüência $\left(e_{n}\right)_{n} \operatorname{com} e_{n}=\left(\delta_{n j}\right)_{j}$ é uma base de Schauder, nos referimos a $\left(e_{n}\right)_{n}$ como sendo a base canônica do espaço em questão e denotaremos por $e_{n}^{\prime}$ o $n$-ésimo funcional coordenado relativo à base $\left(e_{n}\right)_{n}$. 
Observação 1.0.4. Em geral, a norma incondicional multiplicador limitado relativa à uma base $\left(x_{n}\right)_{n}$ não coincide com a norma original. Em $c_{0}$, consideremos a seqüência $\left(x_{n}\right)_{n}$ definida por $x_{1}=e_{1}+e_{2}$ e $x_{n}=e_{n}$ para cada $n \geq 2$. Pelo teorema 4.2.32 de [30] temos que $\left(x_{n}\right)_{n}$ é uma base incondicional em $c_{0}$, pois $e_{1}=x_{1}-x_{2}$. Do fato das seqüências $\left(e_{n}\right)_{n}$ e $\left(x_{n}\right)_{n}$ serem bases incondicionais normalizadas, segue que a base $\left(x_{n}\right)_{n}$ é equivalente à base $\left(e_{n}\right)_{n}$. O vetor $x_{1}-x_{2} \in B_{c_{0}}$ pois $\left\|x_{1}-x_{2}\right\|_{0}=1$, mas $x_{1}-x_{2} \notin B_{b m u\left(x_{n}\right)\left(c_{0}\right)}$ pois $\left\|x_{1}+x_{2}\right\|_{0}=2$, ou seja, $\left\|x_{1}-x_{2}\right\|_{b m u\left(x_{n}\right)} \geq 2$.

Relembramos que um espaço de Banach $E$ é de Schur se para cada seqüência $\left(x_{n}\right)_{n} \subset E$ com $x_{n} \stackrel{\omega}{\longrightarrow} 0$ tem-se que $\left\|x_{n}\right\| \stackrel{n \rightarrow \infty}{\longrightarrow} 0$. O espaço $l_{1}$ é um clássico exemplo de espaço de Schur.

No que segue vamos definir outras propriedades de espaços de Banach, as quais serão utilizadas neste trabalho, e suas principais relações.

Neste trabalho optamos não citar todos os trabalhos originais que tratam das propriedades de Schur, polinomialmente Schur e de Dunford-Pettis devido a vasta bibliografia necessária. A. Hissadomi em sua dissertação de mestrado estudou tais propriedades e então decidimos indicar o seu trabalho, [25], onde pode-se obter informações (e/ou consultar) sobre os trabalhos originais.

Definições 1.0.5. Seja E um espaço de Banach.

a) Dizemos que E tem a propriedade de Dunford-Pettis se, dados qualquer espaço de Banach $F$ e qualquer $T \in \mathcal{L}(E ; F)$ fracamente compacta, $T$ aplica seqüências fracamente de Cauchy em seqüências convergentes. Equivalentemente, se para quaisquer seqüências $\left(x_{n}\right)_{n} \subset E e\left(x_{n}^{*}\right)_{n} \subset E^{\prime}$ com $x_{n} \stackrel{\omega}{\longrightarrow} 0$ e $x_{n}^{*} \stackrel{\omega}{\longrightarrow} 0$ em $E$ e $E^{\prime}$ respectivamente, tem-se que $\lim _{n \rightarrow \infty} x_{n}^{*}\left(x_{n}\right)=0$ (ver [25] Teorema 3.2).

b) Dizemos que E é polinomialmente Schur se para cada seqüência $\left(x_{n}\right)_{n} \subset E$ com $P\left(x_{n}\right) \stackrel{n \rightarrow \infty}{\longrightarrow} 0$ para cada $P \in \mathcal{P}(E)$ tem-se que $\left\|x_{n}\right\| \stackrel{n \rightarrow \infty}{\longrightarrow} 0$.

Segue diretamente da definição, que se o espaço de Banach $E$ é de Schur então $E$ tem a propriedade de Dunford-Pettis. 
Dentre os resultados envolvendo estas definições, destacamos os seguintes:

Proposição 1.0.6. Seja E um espaço de Banach.

1. Se $E^{\prime}$ tem a propriedade de Dunford-Pettis então E também tem a propriedade de Dunford-Pettis (ver [25], Corolário 3.7).

2. $E^{\prime}$ é de Schur se e somente se E tem a propriedade de Dunford-Pettis e $l_{1} \nrightarrow E$ (ver [25], Teorema 3.11).

3. Se E tem a propriedade de Dunford-Pettis então $\mathcal{P}\left({ }^{N} E\right)=\mathcal{P}_{\text {wsc }}\left({ }^{N} E\right) \forall N \in \mathbb{N}$ (ver [25], Corolário 3.46).

4. E é de Schur se e somente se E tem a propriedade de Dunford-Pettis e é polinomialmente Schur (ver [25], Teorema 4.19).

\subsection{Espaços de Banach Especiais}

Nesta seção vamos apresentar alguns espaços de Banach particulares que usaremos nos capítulos 2 e 3. Optamos por apresentá-los aqui, e não no momento da citação, devido à necessidade de se introduzir algumas notações para as suas definições.

\subsubsection{Espaço de Stegall}

Para simplificar, vamos denominar de espaço de Stegall o espaço apresentado por Stegall em [38]. É o primeiro exemplo conhecido de um espaço de Banach com a propriedade de Schur cujo dual não tem a propriedade de Dunford-Pettis.

Primeiramente, vamos introduzir uma notação para simplificar as notações.

Seja $s: \mathbb{N}_{0} \longrightarrow \mathbb{N}_{0}$ definida por: $\left\{\begin{array}{l}s(0)=0 \\ s(n)=1+2+\cdots+n \forall n \in \mathbb{N}\end{array}\right.$ 
Para cada $n \in \mathbb{N}$, definamos

$$
I(n):=\{l \in \mathbb{N}: s(n-1)+1 \leq l \leq s(n)\}=[s(n-1)+1, s(n)] \cap \mathbb{N} .
$$

O espaço de Stegall é definido como:

$E=\left(\bigoplus_{n=1}^{\infty} l_{2}^{(n)}\right)_{1}$, ou seja, $E=\left\{x=\left(x_{i}\right)_{i} \in \mathbb{K}^{\mathbb{N}}: \sum_{n=1}^{\infty}\left(\sum_{i \in I(n)}\left|x_{i}\right|^{2}\right)^{\frac{1}{2}}<\infty\right\}$ munido da norma $\|x\|_{1}=\sum_{n=1}^{\infty}\left(\sum_{i \in I(n)}\left|x_{i}\right|^{2}\right)^{\frac{1}{2}}$.

Com uma demonstração bastante técnica, utilizando-se do teorema 4.2.32 de [30], a seqüência $\left(e_{n}\right)_{n}$ é uma base de Schauder incondicional para o espaço $\left(\bigoplus_{n=1}^{\infty} l_{2}^{(n)}\right)_{1}$.

O espaço de Stegall admite como dual o espaço

$E^{\prime}=\left(\bigoplus_{n=1}^{\infty} l_{2}^{(n)}\right)_{\infty}$, ou seja, $E^{\prime}=\left\{x=\left(x_{i}\right)_{i} \in \mathbb{K}^{\mathbb{N}}: \sup _{n}\left(\sum_{i \in I(n)}\left|x_{i}\right|^{2}\right)^{\frac{1}{2}}<\infty\right\}$ munido da norma $\|x\|_{\infty}=\sup _{n}\left(\sum_{i \in I(n)}\left|x_{i}\right|^{2}\right)^{\frac{1}{2}}$.

E como predual, o espaço

$F=\left(\bigoplus_{n=1}^{\infty} l_{2}^{(n)}\right)_{0}$, ou seja, $F=\left\{y=\left(y_{i}\right)_{i} \in \mathbb{K}^{\mathbb{N}}: \sup _{n \geq k}\left(\sum_{i \in I(n)}\left|y_{i}\right|^{2}\right)^{\frac{1}{2}} \stackrel{k \rightarrow \infty}{\longrightarrow} 0\right\}$ munido da norma $\|y\|_{0}=\sup _{n}\left(\sum_{i \in I(n)}\left|y_{i}\right|^{2}\right)^{\frac{1}{2}}$.

Também não é complicado verificar que a seqüência $\left(e_{n}\right)_{n}$ é uma base de Schauder incondicional para $\left(\left(\bigoplus_{n=1}^{\infty} l_{2}^{(n)}\right)_{0},\|\cdot\|_{0}\right)$. E pelo teorema 1.0.2 temos que a seqüência $\left(e_{n}\right)_{n}$ é uma base de Schauder contrátil para o espaço $\left(\left(\bigoplus_{n=1}^{\infty} l_{2}^{(n)}\right)_{0},\|\cdot\|_{0}\right)$ pois seus coeficientes funcionais associados formam uma base (a base canônica) para o espaço $\left(\left(\bigoplus_{n=1}^{\infty} l_{2}^{(n)}\right)_{1},\|\cdot\|_{1}\right)$ 


\subsubsection{Espaço de Baernstein}

Um espaço de Banach onde cada seqüência limitada admite uma subseqüência cuja média aritmética é convergente é dito que satisfaz a propriedade Banach-Saks.

O espaço de Baernstein, apresentado em [10], é o primeiro espaço de Banach reflexivo conhecido que não satisfaz a propriedade Banach-Saks cujo dual satisfaz a propriedade Banach-Saks, provado por Seifert em [36].

Vamos denotar por $\gamma$ um conjunto não vazio de naturais tal que a cardinalidade de $\gamma$ é menor ou igual ao menor elemento de $\gamma$.

Seja $\Gamma=\{\gamma \subset \mathbb{N},|\gamma| \leq \min \gamma\}$, onde $|\gamma|$ significa a cardinalidade de $\gamma$.

Antes de apresentar o espaço de Baernstein, vamos introduzir algumas notações que serão utilizados. Dados $\gamma, \gamma^{\prime} \in \Gamma$, dizemos que $\gamma \preceq \gamma^{\prime}$ se $\max \gamma<\min \gamma^{\prime}$.

Para cada $x=\left(x_{i}\right)_{i} \in \mathbb{K}^{\mathbb{N}}$, definimos $\sigma(x, \gamma)=\sum_{i \in \gamma}\left|x_{i}\right|$. Para cada $\left\{\gamma_{k}\right\}_{k} \subset \Gamma$, com $\gamma_{k} \preceq \gamma_{k+1}$, definimos:

$$
\sigma\left(x,\left\{\gamma_{k}\right\}_{k}\right)=\left(\sum_{k=1}^{\infty} \sigma\left(x, \gamma_{k}\right)^{2}\right)^{\frac{1}{2}}=\left(\sum_{k=1}^{\infty}\left[\sum_{i \in \gamma_{k}}\left|x_{i}\right|\right]^{2}\right)^{\frac{1}{2}}
$$

O espaço de Baernstein $\mathbb{B}_{\mathbf{2}}$ é o espaço de Banach

$$
\mathbb{B}_{\mathbf{2}}=\left\{x=\left(x_{i}\right)_{i} \in \mathbb{K}^{\mathbb{N}}: \sup _{\left\{\gamma_{k}\right\}_{k} \subset \Gamma, \gamma_{k} \preceq \gamma_{k+1}} \sigma\left(x,\left\{\gamma_{k}\right\}_{k}\right)<\infty\right\}
$$

munido da norma:

$$
\|x\|=\sup _{\left\{\gamma_{k}\right\}_{k} \subset \Gamma, \gamma_{k} \preceq \gamma_{k+1}} \sigma\left(x,\left\{\gamma_{k}\right\}_{k}\right)=\sup _{\left\{\gamma_{k}\right\}_{k} \subset \Gamma, \gamma_{k} \preceq \gamma_{k+1}}\left(\sum_{k=1}^{\infty}\left[\sum_{i \in \gamma_{k}}\left|x_{i}\right|\right]^{2}\right)^{\frac{1}{2}} .
$$

Observamos que $\left\{\gamma_{k}\right\}_{k}, \gamma_{k}=\{k\}$, é uma seqüência de $\Gamma$ com $\gamma_{k} \preceq \gamma_{k+1}$. Assim, dado $x=\left(x_{i}\right)_{i} \in \mathbb{B}_{\mathbf{2}}$, temos que $\left(\sum_{k=1}^{\infty}\left|x_{k}\right|^{2}\right)^{\frac{1}{2}} \leq\|x\|$, ou seja, $x \in l_{2}$.

$\operatorname{Logo} i:\left(\mathbb{B}_{2},\|\cdot\|\right) \hookrightarrow\left(l_{2},\|\cdot\|_{2}\right)$ (a aplicação inclusão) é contínua. 
Com argumentos simples, vemos que a seqüência $\left(e_{n}\right)_{n} \subset \mathbb{B}_{2}$. Em [10], Baernstein demonstra que a seqüência $\left(e_{n}\right)_{n}$ é uma base de Schauder contrátil e limitadamente completa para $\mathbb{B}_{2}$. E desta maneira, o espaço $\mathbb{B}_{2}$ é reflexivo. Observamos também que a seqüência $\left(e_{n}\right)_{n}$ é uma base de Schauder incondicional para o espaço $\mathbb{B}_{\mathbf{2}}$.

Da maneira como foi definido o espaço $\mathbb{B}_{\mathbf{2}}$, destacamos que este espaço nada mais é que o completamento de $c_{00}$ em relação à norma $\|\cdot\|_{B}$ dada por

$\|x\|_{B}=\sup _{\gamma_{1} \preceq \ldots \_\gamma_{n}, n \in \mathbb{N}, \gamma_{k} \in \Gamma}\left(\sum_{k=1}^{n}\left(\sum_{i \in \gamma_{k}}\left|x_{i}\right|\right)^{2}\right)^{\frac{1}{2}}$ para cada $x=\left(x_{i}\right)_{i} \in c_{00}$.

Dado $p, 1<p<\infty$, na construção de $\mathbb{B}_{\mathbf{2}}$, trocando o número 2 por $p$, temos um espaço de Banach que denoteremos por $\mathbb{B}_{\mathbf{p}}$, tal que: $\mathbb{B}_{\mathbf{p}}$ é reflexivo, a seqüência $\left(e_{n}\right)_{n}$ é uma base de Schauder incondicional, $\mathbb{B}_{\mathbf{p}}$ não satisfaz a propriedade de Banach-Saks mas o seu dual satisfaz (ver [18] teorema $0.15 \operatorname{pg} 7)$. E a aplicação inclusão $i:\left(\mathbb{B}_{\mathbf{p}},\|\cdot\|\right) \hookrightarrow\left(l_{p},\|\cdot\|_{p}\right)$ é contínua.

\subsubsection{Espaço de Lorentz}

Considere dados p, $1 \leq p<\infty$, e $w=\left(w_{i}\right)_{i}$ uma seqüência decrescente de reais positivos tais que $w_{1}=1, \lim _{i \rightarrow \infty} w_{i}=0$ e $\sum_{i=1}^{\infty} w_{i}=\infty$, ou seja, $w \in c_{0} \backslash l_{1}$.

O espaço de seqüência de Lorentz $d(w ; p)$ é o espaço de Banach de todas as seqüências $x=\left(x_{i}\right)_{i} \in c_{0}$ tais que $\sup _{\pi \in \sigma}\left(\sum_{i=1}^{\infty}\left|x_{\pi(i)}\right|^{p} \cdot w_{i}\right)^{1 / p}<\infty$ onde $\sigma$ é o conjunto das permutações em $\mathbb{N}$, munido da norma $\|x\|=\sup _{\pi \in \sigma}\left(\sum_{i=1}^{\infty}\left|x_{\pi(i)}\right|^{p} \cdot w_{i}\right)^{1 / p}$.

Originalmente, este espaço aparece nos trabalhos de Garling em [20,21], mas a sua notação é um pouco carregada. Atualmente, este espaço aparece com uma linguagem um pouco distinta, mais simplificada. Sugerimos também ver [34] capítulo 1 a respeito deste espaço, pois L.P. Rodrigues em sua tese apresenta o espaço de Lorentz com a notação atual.

O espaço $d(w ; p)$ é reflexivo se e somente se $p>1$. E a seqüência $\left(e_{n}\right)_{n}$ é uma base de 
Schauder incondicional e limitadamente completa para o espaço $d(w ; p), 1 \leq p<\infty$ ([20], teorema 9 e [12], teorema 2.1).

Dada uma seqüência $\left(x_{n}\right)_{n}$, vamos denotar por $\left(\hat{x}_{n}\right)_{n}$ o rearranjo decrescente da seqüência $\left(\left|x_{n}\right|\right)_{n}$

No caso $p>1$, o espaço $d(w ; p)$ admite como dual (ver teorema 1 em [21]) o espaço

$$
d_{*}(w ; p)=\left\{x=\left(x_{n}\right)_{n} \in c_{0}: \inf \sup _{n} \frac{\sum_{i=1}^{n} \hat{x}_{i}}{\sum_{i=1}^{n}\left|y_{i}\right| w_{i}^{\frac{1}{p}}}<\infty\right\}
$$

onde o ínfimo é tomado sobre todos $y=\left(y_{i}\right)_{i} \in l_{q}$ com $\|y\|_{q}=1$ onde $\frac{1}{p}+\frac{1}{q}=1$, munido da norma $\|x\|=\inf \sup _{n} \frac{\sum_{i=1}^{n} \hat{x}_{i}}{\sum_{i=1}^{n}\left|y_{i}\right| w_{i}^{\frac{1}{p}}}$.

O espaço $d(w ; 1)$ admite como dual (ver [20] teorema 11$)$ o espaço

$$
d^{*}(w ; 1)=\left\{x=\left(x_{n}\right)_{n} \in c_{0}: \sup _{k} \frac{\sum_{n=1}^{k} \hat{x}_{n}}{\sum_{n=1}^{k} w_{n}}=0\right\}
$$

munido da norma $\|x\|=\sup _{k \in \mathbb{N}} \frac{\sum_{n=1}^{k} \hat{x}_{n}}{\sum_{n=1}^{k} w_{n}}$.

Também admite como predual (ver [34] teorema 1.15) o espaço

$$
d_{*}(w ; 1)=\left\{x=\left(x_{n}\right)_{n} \in c_{0}: \lim _{k \rightarrow \infty} \frac{\sum_{n=1}^{k} \hat{x}_{n}}{\sum_{n=1}^{k} w_{n}}=0\right\}
$$

onde a norma é a induzida de $d^{*}(w, 1)$.

A seqüência $\left(e_{n}\right)_{n}$ é uma base de Schauder incondicional e contrátil para o espaço $d_{*}(w ; 1)$, pois $d_{*}(w ; 1)=\overline{\left[e_{n}: n \in \mathbb{N}\right]^{d^{*}(w ; 1)}}($ ver [34], teorema 1.15).

Em $d(w ; 1)$, seja $N \in \mathbb{N}$ tal que $w_{N+1}<w_{N}$ (existe pois a seqüência $w=\left(w_{n}\right)_{n}$ é decrescente). Então, $\left\|\sum_{i=1}^{N+1} e_{i}\right\|=\sum_{i=1}^{N+1} w_{i}<N+1$, e daí, pelo teorema 4.3.6 de [30] temos que $\left(e_{n}\right)_{n}$ não é equivalente à base canônica de $l_{1}$, e conseqüentemente, $e_{n} \stackrel{\omega}{\longrightarrow} 0$ em $d(w ; 1)$. Também temos que o espaço $d^{*}(w ; 1)$ não é separável (ver corolário 2.1 em [12]). 


\section{Capítulo 2}

\section{Espaços que possuem as \\ propriedades $(\mathrm{P})$ e $(\mathrm{RP})$}

Neste capítulo temos por objetivo estudar as propriedades (P) e (RP), definidas por Aron, Choi e Llavona em [4]. Estas propriedades polinomiais foram tema de nossa dissertação de mestrado [39] e, na ocasião, surgiram várias questões relacionadas com estas propriedades que mencionaremos no decorrer do capítulo. Isto nos motivou seguir estudando estas propriedades e suas relações com as propriedades geométricas dos espaços de Banach em questão.

Na seção 1 vamos apresentar exemplos de espaços de Banach e de funções $g$ definidas entre as bolas unitárias fechadas do mesmo tais que as funções $g$ satisfaçam a propriedade ACL (definida em 2.1). Estudaremos a propriedade (P) na seção 2 e apresentaremos novos exemplos de espaços de Banach com a propriedade (P). E na seção 3 vamos estudar a propriedade (RP) apresentando novos exemplos de espaços de Banach que não possuem a propriedade $(\mathbf{R P})$. 


\subsection{Funções com a propriedade ACL}

Sejam $E$ e $F$ espaços de Banach. Em [4], Aron, Choi e Llavona estudaram condições sobre os espaços $E$ e $F$ para que uma função $g: B_{E} \longrightarrow B_{F}$ que satisfaça a seguinte propriedade, que denominaremos ACL:

ACL: Para qualquer polinômio contínuo $P: F \longrightarrow \mathbb{K}, P \circ g: B_{E} \longrightarrow \mathbb{K}$ é uniformemente contínua,

seja necessariamente uniformemente contínua.

Eles mostram que no caso $E=F=c_{0}$, é possível exibir uma função que satisfaz a propriedade ACL e não é uniformemente contínua.

Estudando o espaço de Stegall, observamos também que no seu predual, $E=F=$ $\left(\bigoplus_{n=1}^{\infty} l_{2}^{(n)}\right)_{0}$, é possível exibir uma função que satisfaz a propriedade ACL e é não uniformente contínua. Tal exemplo apresentamos no 59o Seminário Brasileiro de Análise, $[40]$.

Apresentaremos a seguir condições em um espaço de Banach $E$ onde é possível exibir funções $g$ definidas entre as bolas unitárias que satisfaçam a propriedade ACL e não sejam uniformemente contínuas. Isto nos permitirá exibir uma gama de exemplos de espaços e funções que satisfazem a propriedade ACL e não são uniformemente contínuas.

Para exibir uma função com tal propriedade, vamos nos valer fortemente do seguinte conhecido lema.

Lema 2.1.1. Sejam $E, F$ espaços de Banach. Então $f: E \longrightarrow F$ é uniformemente contínua se e somente se para quaisquer duas seqüências $\left(x_{n}\right)_{n},\left(y_{n}\right)_{n}$ em E com $\left\|x_{n}-y_{n}\right\| \stackrel{n \rightarrow \infty}{\longrightarrow} 0$ temos que $\left\|f\left(x_{n}\right)-f\left(y_{n}\right)\right\| \stackrel{n \rightarrow \infty}{\longrightarrow} 0$.

Na seguinte proposição vamos considerar $E$ um espaço de Banach com uma base de Schauder incondicional $\left(x_{n}\right)_{n}$. Muniremos E com a norma $\|\cdot\|_{b m u\left(x_{n}\right)}$ dada na definição 1.0.3, consideremos $I d_{E}:\left(E,\|\cdot\|_{b m u\left(x_{n}\right)}\right) \longrightarrow(E,\|\cdot\|)$ e vamos denotar por $B_{b m u\left(x_{n}\right)(E)}=\left\{x \in E:\|x\|_{b m u\left(x_{n}\right)} \leq 1\right\}$. Como conseqüência temos o seguinte resultado: 
Proposição 2.1.2. Seja E um espaço de Banach com uma base de Schauder contrátil e incondicional $\left(x_{n}\right)_{n}$. Seja $g: B_{b m u\left(x_{n}\right)(E)} \longrightarrow B_{b m u\left(x_{n}\right)(E)}$ a função dada por :

$$
g(x)=\sum_{n=1}^{\infty}\left\|x_{n}\right\|^{n-1} \alpha_{n}^{n} x_{n} \text { para cada } x=\sum_{n=1}^{\infty} \alpha_{n} x_{n} \in B_{b m u\left(x_{n}\right)(E)} .
$$

As seguintes afirmações valem:

1. A função g não é uniformemente contínua em relação a norma original de E.

2. Para quaisquer duas seqüências $\left(u_{j}\right)_{j},\left(v_{j}\right)_{j} \subset B_{b m u\left(x_{n}\right)(E)}$ com $\left\|u_{j}-v_{j}\right\| \stackrel{j \rightarrow \infty}{\longrightarrow 0}$ temos que $g\left(u_{j}\right)-g\left(v_{j}\right) \stackrel{\omega}{\longrightarrow} 0$.

3. Para cada $m \in \mathbb{N}$ e cada $P \in \mathcal{P}_{w u}\left({ }^{m} E\right)$ temos que $P\left(g\left(u_{j}\right)\right)-P\left(g\left(v_{j}\right)\right) \stackrel{j \rightarrow \infty}{\longrightarrow}$ sempre que $\left\|u_{j}-v_{j}\right\| \stackrel{j \rightarrow \infty}{\longrightarrow} 0 \operatorname{com}\left(u_{j}\right)_{j},\left(v_{j}\right)_{j} \subset B_{b m u\left(x_{n}\right)(E)}$. Além disso, se $\mathcal{P}\left({ }^{m} E\right)=\mathcal{P}_{w s c}\left({ }^{m} E\right)$ para cada $m \in \mathbb{N}$ temos que $P\left(g\left(u_{j}\right)\right)-P\left(g\left(v_{j}\right)\right) \stackrel{j \rightarrow \infty}{\longrightarrow}$ para cada $P \in \mathcal{P}\left({ }^{m} E\right)$ e cada $m \in \mathbb{N}$. Em particular, se $\left(x_{n}\right)_{n}$ é tal que a aplicação Id $d_{E}$ é uma isometria, segue que g satisfaz a propriedade $\mathrm{ACL}$.

Demonstração: Primeiramente vamos mostrar que a série que define a função $g$ converge e a imagem de $g$ está contida na bola. De fato: Seja $x=\sum_{n=1}^{\infty} \alpha_{n} x_{n} \in B_{b m u\left(x_{n}\right)(E)}$ com $x \neq 0$ e provemos que $\sum_{n=1}^{\infty}\left\|x_{n}\right\|^{n-1} \alpha_{n}^{n} x_{n}$ converge. Para cada $n \in \mathbb{N}$ tomemos a seqüência $\left(\beta_{i}\right)_{i} \operatorname{com} \beta_{i}=\delta_{i n}$. Então, por definição de $\|\cdot\|_{b m u\left(x_{n}\right)}$ temos que $\left\|\alpha_{n} x_{n}\right\|=\left\|\sum_{i=1}^{\infty} \beta_{i} \alpha_{i} x_{i}\right\| \leq\|x\|_{b m u\left(x_{n}\right)} \leq 1$ e assim $\left|\alpha_{n}^{n-1}\right|\left\|x_{n}\right\|^{n-1} \leq 1$. Agora, tomemos a seqüência $\left(\alpha_{n}^{n-1}\left\|x_{n}\right\|^{n-1}\right)_{n}$. Então $\left(\alpha_{n}^{n-1}\left\|x_{n}\right\|^{n-1}\right)_{n} \in B_{l_{\infty}}$ e conseqüentemente, $\sum_{n=1}^{\infty} \alpha_{n}^{n-1}\left\|x_{n}\right\|^{n-1} \alpha_{n} x_{n}$ é convergente, pois $\left(x_{n}\right)_{n}$ é uma base incondicional, ou seja, $\sum_{n=1}^{\infty}\left\|x_{n}\right\|^{n-1} \alpha_{n}^{n} x_{n}$ é convergente (ver resultado $4.2 .8 \mathrm{em}[30]$ ).

Também $\left\|\sum_{n=1}^{\infty}\right\| x_{n}\left\|^{n-1} \alpha_{n}^{n} x_{n}\right\|_{b m u\left(x_{n}\right)} \leq\left\|\left(\alpha_{n}^{n-1}\left\|x_{n}\right\|^{n-1}\right)_{n}\right\|_{\infty}\left\|\sum_{n=1}^{\infty} \alpha_{n} x_{n}\right\|_{b m u\left(x_{n}\right)} \leq 1$ (ver proposição 4.2 .17 em [30]). Agora, se $x=0$ temos que $g(0)=0$ e portanto $g(x) \in B_{b m u\left(x_{n}\right)(E)}$ para cada $x \in B_{b m u\left(x_{n}\right)(E)}$.

(1) Tomemos as seqüências $\left(z_{n}\right)_{n}$ e $\left(y_{n}\right)_{n}$ onde $z_{n}=\frac{1}{\left\|x_{n}\right\|} x_{n}$ e $y_{n}=\left(1-\frac{1}{n}\right) z_{n}$. Podemos reescrever $z_{n}=\sum_{i=1}^{\infty} \frac{1}{\left\|x_{n}\right\|} \delta_{n i} x_{i}$ para cada $n \in \mathbb{N}$. Para cada $\left(\beta_{i}\right)_{i} \in S_{l_{\infty}}$ temos que 
$\left\|\sum_{i=1}^{\infty} \beta_{i} \frac{1}{\left\|x_{n}\right\|} \delta_{n i} x_{i}\right\| \leq\left\|\frac{x_{n}}{\left\|x_{n}\right\|}\right\|=1$. E por definição da norma $\|\cdot\|_{b m u\left(x_{n}\right)}$, temos que
$\left\|z_{n}\right\|_{b m u\left(x_{n}\right)} \leq 1$, ou seja, $z_{n} \in B_{b m u\left(x_{n}\right)(E)}$.

Também $\left\|y_{n}\right\|_{b m u\left(x_{n}\right)}=\left|1-\frac{1}{n}\right|\left\|z_{n}\right\|_{b m u\left(x_{n}\right)} \leq 1-\frac{1}{n}<1$, ou seja, $y_{n} \in B_{b m u\left(x_{n}\right)(E)}$.

Também $\left\|z_{n}-y_{n}\right\|_{b m u\left(x_{n}\right)}=\frac{1}{n}\left\|z_{n}\right\|_{b m u\left(x_{n}\right)} \leq \frac{1}{n} \stackrel{n \rightarrow \infty}{\longrightarrow} 0$. Mas

$$
\begin{aligned}
& \left\|g\left(z_{n}\right)-g\left(y_{n}\right)\right\|_{b m u\left(x_{n}\right)} \geq\left\|g\left(z_{n}\right)-g\left(y_{n}\right)\right\|= \\
& \|\| x_{n}\left\|^{n-1} \frac{1}{\left\|x_{n}\right\|^{n}} x_{n}-\right\| x_{n}\left\|^{n-1}\left(1-\frac{1}{n}\right)^{n} \frac{1}{\left\|x_{n}\right\|^{n}} x_{n}\right\|= \\
& \left\|\left(1-\left(1-\frac{1}{n}\right)^{n}\right) \frac{1}{\left\|x_{n}\right\|} x_{n}\right\|=\left|1-\left(1-\frac{1}{n}\right)^{n}\right| \stackrel{n \rightarrow \infty}{\longrightarrow}\left|1-e^{-1}\right| .
\end{aligned}
$$

Agora, pelo lema 2.1.1 temos que $g$ não é uniformemente contínua em relação à norma $\|\cdot\|_{b m u\left(x_{n}\right)}$. Como as normas $\|\cdot\|$ e $\|\cdot\|_{b m u\left(x_{n}\right)}$ são equivalentes, temos que $g$ não é uniformemente contínua em relação a norma orginal de $E$.

(2) Sejam $\left(u_{j}\right)_{j},\left(v_{j}\right)_{j} \subset B_{b m u\left(x_{n}\right)(E)}$ duas seqüências tais que $\left\|u_{j}-v_{j}\right\| \stackrel{j \rightarrow \infty}{\longrightarrow} 0$. Sendo $\left(x_{n}\right)_{n}$ uma base contrátil, basta mostrar que $x_{n}^{\prime}\left(g\left(u_{j}\right)-g\left(v_{j}\right)\right) \stackrel{j \rightarrow \infty}{\longrightarrow} 0$ para cada funcional coordenado $x_{n}^{\prime}$. Para cada $j \in \mathbb{N}, u_{j}=\sum_{i=1}^{\infty} u_{j i} x_{i}$ e $v_{j}=\sum_{i=1}^{\infty} v_{j i} x_{i}$. Seja $\epsilon>0$ e dado $n \in \mathbb{N}$ fixo, tomemos $\epsilon^{\star}=\frac{\left\|x_{n}\right\| \epsilon}{n}$. Como as normas $\|\cdot\|$ e $\|\cdot\|_{b m u\left(x_{n}\right)}$ são equivalentes, existe $j_{0} \in \mathbb{N}$ tal que $\left\|u_{j}-v_{j}\right\|_{b m u\left(x_{n}\right)}<\epsilon^{\star}$ para cada $j \geq j_{0}$. Para cada $j \in \mathbb{N}$ temos que

$$
\left|x_{n}^{\prime}\left(g\left(u_{j}\right)-g\left(v_{j}\right)\right)\right|=\left|x_{n}^{\prime}\left(\sum_{i=1}^{\infty}\left\|x_{i}\right\|^{i-1}\left(u_{j i}^{i}-v_{j i}^{i}\right) x_{i}\right)\right|=\left\|x_{n}\right\|^{n-1}\left|u_{j n}^{n}-v_{j n}^{n}\right| .
$$

$\operatorname{Mas}\left|u_{j n}^{n}-v_{j n}^{n}\right|=\left|u_{j n}-v_{j n}\right| \cdot\left|u_{j n}^{n-1} v_{j n}^{0}+u_{j n}^{n-2} v_{j n}+\ldots+u_{j n} v_{j n}^{n-2}+u_{j n}^{0} v_{j n}^{n-1}\right|$

$\leq\left|u_{j n}-v_{j n}\right| \cdot\left[\left|u_{j n}^{n-1}\right|\left|v_{j n}^{0}\right|+\left|u_{j n}^{n-2}\right|\left|v_{j n}+\ldots+\right| u_{j n}|| v_{j n}^{n-2}|+| u_{j n}^{0}|| v_{j n}^{n-1} \mid\right] \mathrm{e}$ como $\left\|u_{j n} x_{n}\right\| \leq\left\|u_{j}\right\|_{b m u\left(x_{n}\right)} \leq 1,\left\|v_{j n} x_{n}\right\| \leq\left\|v_{j}\right\|_{b m u\left(x_{n}\right)} \leq 1 \mathrm{e}$ $\left\|\left(u_{j n}-v_{j n}\right) x_{n}\right\| \leq\left\|u_{j}-v_{j}\right\|_{b m u\left(x_{n}\right)}$, temos que

$$
\left|x_{n}^{\prime}\left(g\left(u_{j}\right)-g\left(v_{j}\right)\right)\right| \leq\left\|x_{n}\right\|^{n-1}\left|u_{j n}-v_{j n}\right| \frac{n}{\left\|x_{n}\right\|^{n-1}} \leq \frac{n}{\left\|x_{n}\right\|}\left\|u_{j}-v_{j}\right\|_{b m u\left(x_{n}\right)} .
$$

Logo, para cada $j \geq j_{0},\left|x_{n}^{\prime}\left(g\left(u_{j}\right)-g\left(v_{j}\right)\right)\right| \leq \epsilon$. Sendo $\left(x_{n}\right)_{n}$ uma base contrátil, temos que $E^{\prime}=\left[\left(x_{n}^{\prime}\right)_{n}\right]$. E assim, $g\left(u_{j}\right)-g\left(v_{j}\right) \stackrel{\omega}{\longrightarrow} 0$.

(3) Sejam $\left(u_{j}\right)_{j},\left(v_{j}\right)_{j} \subset B_{b m u\left(x_{n}\right)(E)}$ tais que $\left\|u_{j}-v_{j}\right\| \stackrel{j \rightarrow \infty}{\longrightarrow} 0$. Seja $\epsilon>0$ e tomemos $W=\left\{g\left(u_{j}\right), g\left(v_{j}\right): j \in \mathbb{N}\right\}$. $W$ é um subconjunto limitado em $E$. Dado $P \in \mathcal{P}_{w u}\left({ }^{m} E\right)$ existem $f_{1}, \ldots, f_{s} \in E^{\prime}$ e $\delta>0$ tais que $|P(x)-P(y)|<\epsilon$ sempre que $\left|f_{i}(x-y)\right|<\delta$ para cada $i \in\{1, \ldots, s\}$ com $x, y \in W$. De (2), para o $\delta$ encontrado acima existe $j_{0} \in \mathbb{N}$ 
tal que $\left|f_{i}\left(g\left(u_{j}\right)-g\left(v_{j}\right)\right)\right|<\delta$ para cada $i \in\{1, \ldots, s\}$ e cada $j \geq j_{0}$. Assim, para cada $j \geq j_{0},\left|P\left(g\left(u_{j}\right)\right)-P\left(g\left(v_{j}\right)\right)\right|<\epsilon$, ou seja, $\left|P\left(g\left(u_{j}\right)\right)-P\left(g\left(v_{j}\right)\right)\right| \stackrel{j \rightarrow \infty}{\longrightarrow} 0$. O espaço $E$ admite base contrátil, e então pelo teorema 4.4 .20 de [30] temos que $l_{1} \hookrightarrow E$, e assim, pela proposição 2.12 de $[7]$ temos que $\mathcal{P}_{w u}\left({ }^{m} E\right)=\mathcal{P}_{w s c}\left({ }^{m} E\right)$ para cada $m \in \mathbb{N}$. Então, se $\mathcal{P}\left({ }^{m} E\right)=\mathcal{P}_{w s c}\left({ }^{m} E\right)$ para cada $m \in \mathbb{N}$, temos que $\left|P\left(g\left(u_{j}\right)\right)-P\left(g\left(v_{j}\right)\right)\right| \stackrel{j \rightarrow \infty}{\longrightarrow} 0$ para cada $P \in \mathcal{P}\left({ }^{m} E\right)$ e cada $m \in \mathbb{N}$. Se além disso, a base $\left(x_{n}\right)_{n}$ é tal que a aplicação $I d_{E}$ é uma isometria, ou seja, $B_{E}=B_{b m u\left(x_{n}\right)(E)}$, temos que $g$ satisfaz a propriedade ACL.

Observação 2.1.3. Sejam E um espaço de Banach com uma base de Schauder contrátil e incondicional $\left(x_{n}\right)_{n}$ e F um espaço de Banach com uma base de Schauder $\left(y_{n}\right)_{n}$ equivalente $\grave{a}$ base $\left(x_{n}\right)_{n}$ de $E$. Então existe um isomorfismo sobrejetor $T: E \longrightarrow F$ tal que $T\left(x_{n}\right)=y_{n} \forall n \in \mathbb{N}$. Dados $\alpha, \beta \in \mathbb{C}$ com $0<|\alpha| \leq 1 e|\beta| \geq\|T\|_{b m u}$ onde $\|T\|_{b m u}=\sup \left\{\|T(x)\|_{b m u\left(y_{n}\right)}:\|x\|_{b m u\left(x_{n}\right)} \leq 1\right\}$, os resultados da proposição 2.1.2 são válidos para a função $g_{(\alpha, \beta)}: B_{b m u\left(x_{n}\right)(E)} \longrightarrow B_{b m u\left(y_{n}\right)(F)}$ definida por :

$$
g_{(\alpha, \beta)}(x)=\sum_{n=1}^{\infty} \frac{\alpha}{\beta}\left\|x_{n}\right\|^{n-1} \alpha_{n}^{n} y_{n} \text { para cada } x=\sum_{n=1}^{\infty} \alpha_{n} x_{n} \in B_{b m u\left(x_{n}\right)(E) .} .
$$

Como conseqüência, podemos observar que em espaços de Banach $E$ com base de Schauder $\left(x_{n}\right)_{n}$ cujo dual é de Schur e tal que $\left(E ;\|\cdot\|_{b m u\left(x_{n}\right)}\right)$ e $(E ;\|\cdot\|)$ são isometricamente isomorfos, é possível exibir funções, definidas entre as bolas unitárias fechadas, não uniformemente contínuas e que satisfazem a propriedade ACL.

Observação 2.1.4. Seja E um espaço de Banach com uma base de Schauder incondicional $\left(x_{n}\right)_{n}$ e suponhamos que $E^{\prime}$ é de Schur. Existe uma família de funções $g: B_{b m u\left(x_{n}\right)(E)} \longrightarrow B_{b m u\left(x_{n}\right)(E)}$ tais que:

1. As funções g não são uniformemente contínuas em relação a norma original de $E$.

2. $P \circ g$ é uniformemente contínua para cada $P \in \mathcal{P}(E)$. Em particular, se $\left(x_{n}\right)_{n}$ é tal que a aplicação $I d_{E}$ é uma isometria, as funções g satisfazem a propriedade ACL.

De fato: Como $E^{\prime}$ é de Schur temos que $l_{1} \nsucc E$, e assim $\left(x_{n}\right)_{n}$ é uma base contrátil, pois a base $\left(x_{n}\right)_{n}$ é incondicional (ver teorema 4.4.21 em [30]). Também temos que 
$\mathcal{P}\left({ }^{m} E\right)=\mathcal{P}_{\text {wsc }}\left({ }^{m} E\right)$ para cada $m \in \mathbb{N}$, pois $E$ tem a propriedade de Dunford-Pettis. Agora, para cada $\alpha, \beta \in \mathbb{C}$ com $0<|\alpha| \leq 1$ e $|\beta| \geq 1$, basta tomar as funções $g_{(\alpha, \beta)}$ como as definidas na observação 2.1.3.

Exemplo 2.1.5. O espaço $E=\left(\bigoplus_{n=1}^{\infty} l_{2}^{(n)}\right)_{0}$ satisfaz as condições da observação 2.1.4 e a aplicação $I d_{E}:\left(E,\|\cdot\|_{0_{b m u\left(e_{n}\right)}}\right) \longrightarrow\left(E,\|\cdot\|_{0}\right)$ é uma isometria. Assim, as funções $g_{(\alpha, \beta)}: B_{E} \longrightarrow B_{E}$ dadas em 2.1.3 satisfazem a propriedade ACL.

Dado um espaço de Banach E com uma base de Schauder contrátil e incondicional $\left(x_{n}\right)$, para garantir que a imagem da função $g: B_{E} \longrightarrow E$ (definida na observação 2.1.3) esteja contida em $B_{E}$, a norma $\|\cdot\|_{b m u\left(x_{n}\right)}$ desempenha um papel fundamental. Por exemplo, em $c_{0}$ consideremos a base $\left(x_{n}\right)_{n}$, onde $x_{1}=e_{1}+e_{2}$ e $x_{n}=e_{n}$ para cada $n \geq 2$, e $g: B_{c_{0}} \longrightarrow c_{0}$ dada por $g(x)=\sum_{n=1}^{\infty} \alpha_{n}^{n} x_{n}$ para cada $x=\sum_{n=1}^{\infty} \alpha_{n} x_{n} \in B_{c_{0}}$. Temos que $x_{1}-x_{2} \in B_{c_{0}}$ mas $g\left(x_{1}-x_{2}\right)=x_{1}+x_{2} \notin B_{c_{0}}$.

A condição da base $\left(x_{n}\right)_{n}$ ser contrátil é necessária na proposição 2.1 .2 como podemos observar a seguir: Dados $\alpha, \beta \in \mathbb{C} \operatorname{com} 0<|\alpha| \leq 1$ e $|\beta| \geq 1$, consideremos a função $g_{(\alpha, \beta)}: B_{l_{1}} \longrightarrow B_{l_{1}}$ como a definida na observação 2.1.3. Ela não satisfaz os itens 2 e 3 da proposição 2.1.2.

De fato: Para as seqüências $\left(e_{n}\right)_{n},\left(f_{n}\right)_{n}, f_{n}=\left(1-\frac{1}{n}\right) e_{n}$, temos que $\left\|e_{n}-f_{n}\right\|_{1}=\frac{1}{n} \stackrel{n \rightarrow \infty}{\longrightarrow} 0$ mas $\left\|g_{(\alpha, \beta)}\left(e_{n}\right)-g_{(\alpha, \beta)}\left(f_{n}\right)\right\| \stackrel{n \rightarrow \infty}{\longrightarrow} \frac{|\alpha|}{|\beta|}\left|1-e^{-1}\right|>0$. Como $l_{1}$ é de Schur, temos que $g_{(\alpha, \beta)}\left(e_{n}\right)-g_{(\alpha, \beta)}\left(f_{n}\right) \stackrel{\omega}{\leftrightarrow} 0$. Também temos que cada polinômio definido sobre $l_{1}$ é fracamente seqüencialmente contínuo pois $l_{1}$ é Schur. A função $g_{(\alpha, \beta)}$ não satisfaz o item 3 , pois para $P(x)=\sum_{i=1}^{\infty} \alpha_{i}^{N}, x=\sum_{i=1}^{\infty} \alpha_{i} e_{i}$, temos que $\left|P\left(g_{(\alpha, \beta)}\left(e_{n}\right)\right)-P\left(g_{(\alpha, \beta)}\left(f_{n}\right)\right)\right|=\frac{|\alpha|^{N}}{|\beta|^{N}}\left|1-\left(1-\frac{1}{n}\right)^{n N}\right| \stackrel{n \rightarrow \infty}{\longrightarrow}|\alpha|^{N}\left|1-e^{-N}\right|>0$.

Tsirelson em [43] descreveu um método de como construir uma classe de espaços com propriedades especiais. Não descreveremos aqui a construção de tais espaços, mas sim as propriedades que vamos precisar. Vamos denotar por $T^{*}$ o espaço original de Tsirelson. O espaço $T^{*}$ é um espaço de Banach reflexivo de dimensão infinita, que não contém subespaços isomorfos a $c_{0}$ ou quaisquer $l_{p}, 1 \leq p<\infty$. A seqüência $\left(e_{n}\right)_{n}$, é uma base de 
Schauder incondicional normalizada para $T^{*}$. Segue da observação 2.b pg 17 de [18] que a aplicação $I d_{T^{*}}:\left(T^{*},\|\cdot\|_{b m u\left(e_{n}\right)}\right) \longrightarrow\left(T^{*},\|\cdot\|\right)$ é uma isometria.

Exemplo 2.1.6. Para cada $\alpha, \beta \in \mathbb{C}$ com $0<|\alpha| \leq 1$ e $|\beta| \geq 1$, a função $g_{(\alpha, \beta)}: B_{T^{*}} \longrightarrow B_{T^{*}}$ (definida na observação 2.1.3) satisfaz a propriedade ACL.

De fato: Alencar, Aron e Dineen em [1] provam que para cada $m \in \mathbb{N}$ tem-se que $\mathcal{P}\left({ }^{m} T^{*}\right)=\mathcal{P}_{w s c}\left({ }^{m} T^{*}\right)$. Sendo $T^{*}$ um espaço reflexivo, $\left(e_{n}\right)_{n}$ é uma base contrátil. Pela proposição 2.1.2 temos que $g_{(\alpha, \beta)}$ não é uniformemente contínua e $P \circ g_{(\alpha, \beta)}$ é uniformemente contínua para cada $P \in \mathcal{P}\left({ }^{m} T^{*}\right)$ e cada $m \in \mathbb{N}$.

Exemplo 2.1.7. Sejam $w=\left(w_{i}\right)_{i} \in c_{0} \backslash l_{1}$ uma seqüência decrescente de reais com $w_{1}=1$ e $1 \leq p<\infty$. No espaço $E=d_{*}(w ; p)$ consideremos a base canônica $\left(e_{n}\right)_{n}$ e para cada $\alpha, \beta \in \mathbb{C} \operatorname{com} 0<|\alpha| \leq 1$ e $|\beta| \geq 1$, tomemos a função $g_{(\alpha, \beta)}: B_{E} \longrightarrow B_{E}$ (definida na observação 2.1.3). São equivalentes:

1. $P \circ g_{(\alpha, \beta)}$ é uniformemente contínua para cada $P \in \mathcal{P}\left({ }^{N} d_{*}(w ; p)\right), N \geq 2$.

2. $1 \leq p<\frac{N}{N-1}$ e $w \notin l_{q} \operatorname{com} q=\frac{N}{N-p(N-1)}$.

De fato: Primeiramente vamos assumir que vale (1) e suponhamos, por absurdo, que (2) não ocorre. Então, ou $p \geq \frac{N}{N-1}$ ou $w \in l_{q} \operatorname{com} q=\frac{N}{N-p(N-1)}$. Vamos denotar por $N^{*}=\frac{N}{N-1}$. Se $p \geq N^{*}$, a aplicação inclusão $i: l_{N^{*}} \hookrightarrow d(w ; p)$ é contínua, pois dado $x=\left(x_{i}\right)_{i} \in l_{N^{*}}$, para cada permutação $\sigma$ de naturais,

$$
\left(\sum_{i=1}^{\infty}\left|x_{\sigma(i)}\right|^{p} w_{i}\right)^{\frac{1}{p}} \leq\left(\sum_{i=1}^{\infty}\left|x_{\sigma(i)}\right|^{p}\right)^{\frac{1}{p}} \leq\left(\sum_{i=1}^{\infty}\left|x_{\sigma(i)}\right|^{N^{*}}\right)^{\frac{1}{N^{*}}}=\|x\|_{N^{*}}<\infty .
$$

E assim, a transposta $i^{t}: d^{*}(w ; p) \longrightarrow l_{N}$ é bem definida $\left(d^{*}(w ; p)\right.$ denota o dual de $\left.d(w ; p)\right)$ e $d_{*}(w ; p) \subset l_{N}$, e conseqüentemente podemos definir o polinômio $P: d_{*}(w ; p) \longrightarrow \mathbb{K}$ por $P(x)=\sum_{i=1}^{\infty} x_{i}^{N}$ para cada $x=\sum_{i=1}^{\infty} x_{i} e_{i} \in d_{*}(w ; p)$. Para as seqüências $\left(e_{n}\right)_{n}$ (base de $\left.d_{*}(w ; p)\right)$ e $\left(f_{n}\right)_{n}$ onde $f_{n}=\left(1-\frac{1}{n}\right) e_{n}$ temos que $\left\|e_{n}-f_{n}\right\| \stackrel{n \rightarrow \infty}{\longrightarrow} 0$. Mas $\mid P\left(g_{(\alpha, \beta)}\left(e_{j}\right)\right)-$ $P\left(g_{(\alpha, \beta)}\left(f_{j}\right)\right)\left|=\frac{|\alpha|^{N}}{|\beta|^{N}}\right| 1-\left(1-\frac{1}{j}\right)^{j N}\left|\stackrel{j \rightarrow \infty}{\longrightarrow} \frac{|\alpha|^{N}}{|\beta|^{N}}\right| 1-e^{-N} \mid>0$, ou seja, $P \circ g_{(\alpha, \beta)}$ não é uniformemente contínua, uma contradição. 
Para o outro caso, $p<N^{*}$ e $w \in l_{q}$, dado $x=\left(x_{i}\right)_{i} \in l_{N^{*}}$, para cada permutação $\sigma$ de naturais, pela desigualdade de Hölder, tem-se que como $q^{*}=\frac{N^{*}}{p}$ (conjugado de $q$ ),

$$
\sum_{i=1}^{\infty}\left|x_{\sigma(i)}\right|^{p} w_{i} \leq\left(\sum_{i=1}^{\infty}\left|x_{\sigma(i)}\right|^{N^{*}}\right)^{\frac{p}{N^{*}}}\left(\sum_{i=1}^{\infty}\left|w_{i}\right|^{q}\right)^{\frac{1}{q}}=\|x\|_{N^{*}}\|w\|_{q}<\infty,
$$

ou seja, $i: l_{N^{*}} \hookrightarrow d(w ; p)$ é contínua e prosseguindo de maneira análoga tem-se uma contradição.

Agora, vamos provar que (2) implica (1). De fato, se $1 \leq p<\frac{N}{N-1}$ e $w \notin l_{q}$ com $q=\frac{N}{N-p(N-1)}$, então, pela proposição 2.4 de [37] temos que $\mathcal{P}\left({ }^{N} d_{*}(w ; p)\right)=\mathcal{P}_{w s c}\left({ }^{N} d_{*}(w ; p)\right)$. Como a aplicação $I d_{d_{*}(w ; p)}:\left(d_{*}(w ; p) ;\|\cdot\|_{b m u\left(e_{n}\right)}\right) \longrightarrow\left(d_{*}(w ; p) ;\|\cdot\|\right)$ é uma isometria (de fato, dado $x=\left(x_{i}\right)_{i} \in d_{*}(w ; p)$, para cada $\left(\beta_{i}\right)_{i} \in S_{l_{\infty}}$ vale $\hat{\beta_{i} x_{i}} \leq \hat{\beta}_{i} \hat{x_{i}} \leq \hat{x_{i}}$ e daí, $\left.\|x\|_{b m u\left(e_{n}\right)} \leq\|x\|\right)$, da proposição 2.1.2 temos que $P \circ g_{(\alpha, \beta)}$ é uniformemente contínua para cada polinômio $P \in \mathcal{P}\left({ }^{N} d_{*}(w ; p)\right)$.

\subsection{A condição $(\star \star)$ e a propriedade $(\mathrm{P})$}

Nesta seção abordaremos a denominada propriedade (P) definida e estabelecida por Aron, Choi e Llavona em [4]. Tal propriedade nos permite classificar espaços de Banach onde funções $g: B_{E} \longrightarrow B_{E}$ que satisfaçam a propriedade ACL sejam uniformemente contínuas.

Sejam $E$ e $F$ espaços de Banach e $g: B_{E} \longrightarrow B_{F}$ uma função que satisfaz a propriedade ACL. Se o espaço $F$ satisfaz a condição $(\star \star)$ :

$(\star \star)$ : Para quaisquer duas seqüências limitadas $\left(u_{n}\right)_{n},\left(v_{n}\right)_{n}$ em $F$ e para cada $m \in \mathbb{N}$ e cada $P \in \mathcal{P}\left({ }^{m} F\right)$ com $\mid P\left(u_{n}\right)-P\left(v_{n}\right) \stackrel{n \rightarrow \infty}{\longrightarrow} 0$ implica que $\left\|u_{n}-v_{n}\right\| \stackrel{n \rightarrow \infty}{\longrightarrow} 0$,

então $g$ é uniformemente contínua.

Com isto podemos concluir:

Proposição 2.2.1. Seja E um espaço de Banach com uma base de Schauder contrátil 
e incondicional $\left(x_{n}\right)_{n}$. Se $\mathcal{P}_{\text {wsc }}\left({ }^{m} E\right)=\mathcal{P}\left({ }^{m} E\right)$ (e neste caso $\mathcal{P}_{f}\left({ }^{m} E\right)$ é denso em $\mathcal{P}\left({ }^{m} E\right)$ ) para cada $m \in \mathbb{N}$, então E não satisfaz a condição $(\star \star)$.

Demonstração: Tomemos $\alpha, \beta \in \mathbb{C} \operatorname{com} 0<|\alpha| \leq 1,|\beta| \geq 1$ e consideremos a função $g_{(\alpha, \beta)}: B_{b m u\left(x_{n}\right)(E)} \longrightarrow B_{b m u\left(x_{n}\right)(E)}$ (definida na observação 2.1.3). Segue da proposição 2.1.2 que a função $g_{(\alpha, \beta)}$ não é uniformemente contínua em relação à norma $\|\cdot\|_{b m u\left(x_{n}\right)}$ e $P \circ g_{(\alpha, \beta)}: B_{b m u\left(x_{n}\right)(E)} \longrightarrow \mathbb{K}$ é uniformemente contínua para cada $P \in \mathcal{P}\left({ }^{m} E\right)$ e cada $m \in \mathbb{N}$. Desta maneira, existem duas seqüências limitadas em $E,\left(u_{n}\right)_{n}$ e $\left(v_{n}\right)_{n}$ tais que $\left|P\left(u_{n}\right)-P\left(v_{n}\right)\right| \stackrel{n \rightarrow \infty}{\longrightarrow} 0$ para cada $P \in \mathcal{P}\left({ }^{m} E\right)$ e cada $m \in \mathbb{N}$ mas $\left\|u_{n}-v_{n}\right\|_{b m u} \stackrel{n \rightarrow \infty}{\rightarrow} 0$. Como as normas $\|\cdot\|$ e $\|\cdot\|_{b m u\left(x_{n}\right)}$ são equivalentes, segue que $\left\|u_{n}-v_{n}\right\| \stackrel{n \rightarrow \infty}{\rightarrow} 0$. Portanto $E$ não satisfaz a condição $(\star \star)$.

Exemplos 2.2.2. Os espaços $\left(\bigoplus_{n=1}^{\infty} l_{2}^{(n)}\right)_{0}, T^{*}$ e $d_{*}(w ; 1)$ se $w \notin l_{N} \forall N \in \mathbb{N}$ satisfazem as condições da proposição, e portanto, não satisfazem a condição ( $\star \star)$.

Para poder determinar sob que condições os espaços de Banach satisfazem a condição $(\star \star)$, Aron, Choi e Llavona em [4] introduziram uma propriedade mais fraca que ( $\star \star)$, e a chamaram de propriedade $(\mathbf{P})$.

Definição 2.2.3. Seja E um espaço de Banach. Dizemos que E tem a propriedade $\mathbf{( P )}$ se para quaisquer duas seqüencias limitadas $\left(u_{j}\right)_{j},\left(v_{j}\right)_{j}$ em E tais que para cada

$n \in \mathbb{N}$ e cada $P \in \mathcal{P}\left({ }^{n} E\right)$ com $\left|P\left(u_{j}\right)-P\left(v_{j}\right)\right| \stackrel{j \rightarrow \infty}{\longrightarrow} 0$ tem-se que $\left|Q\left(u_{j}-v_{j}\right)\right| \stackrel{j \rightarrow \infty}{\longrightarrow} 0$ para cada $Q \in \mathcal{P}\left({ }^{m} E\right)$ e cada $m \in \mathbb{N}$.

A seguir apresentamos um dos principais teoremas, demonstrados em [4] por Aron, Choi e Llavona, que envolvem as relações destas definições com certas propriedades de espaços de Banach.

Teorema 2.2.4 (Aron, Choi e Llavona, [4]). Seja E um espaço de Banach. Considere as seguintes afirmações:

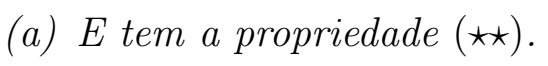


(b) E é polinomialmente Schur.

(c) E tem a propriedade Dunford-Pettis.

(d) E tem a propriedade $(\mathbf{P})$.

Então temos as seguintes implicações: $(\mathbf{a}) \Rightarrow(\mathbf{b}),(\mathbf{a}) \Rightarrow(\mathbf{d}),(\mathbf{c}) \Rightarrow(\mathbf{d})$, (b) e (d) $\Rightarrow$ (a).

Corolário 2.2.5. Seja E um espaço de Banach. Então E é de Schur se e somente se E

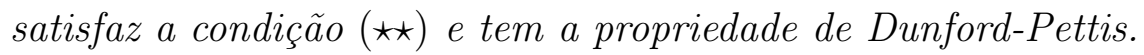

Demonstração: Segue diretamente do teorema 2.2.4, pois um espaço de Banach E é de Schur se e somente se tem a propriedade de Dunford-Pettis e é polinomialmente Schur (proposição 1.0.6(4).

Corolário 2.2.6. Seja E um espaço de Banach. Suponhamos que E admite uma cópia de um espaço de Banach que não é polinomialmente Schur. Então E não satisfaz a condição $(\star \star)$.

Demonstração: Seja $F$ um espaço de Banach tal que $F \hookrightarrow E$ e $F$ não é polinomialmente Schur. Então E também não é polinomialmente Schur. Agora, pelo teorema 2.2.4 tem-se que $E$ não satisfaz a condição $(\star \star)$.

O espaço de Schreier ([18], construção 0.2 pg 1), que vamos denotar por $\mathcal{S}$, é o completamento de $c_{00}$ em relação à norma dada por $\|x\|_{\mathcal{S}}=\sup _{\gamma \in \Gamma} \sum_{i \in \gamma}\left|x_{i}\right|$ para cada $x=\left(x_{i}\right)_{i} \in c_{00}$.

A seqüência $\left(e_{n}\right)_{n}$ é uma base de Schauder contrátil e incondicional para $\mathcal{S}$.

A seqüência $\left(u_{i}\right)_{i}$ definida por:

$u_{1}=e_{1}, u_{2}=\frac{1}{2}\left(e_{2}+e_{3}\right), u_{3}=2^{-2}\left(e_{4}+e_{5}+e_{6}+e_{7}\right), \ldots, u_{n}=2^{1-n}\left(\sum_{k=2^{n-1}}^{2^{n}-1} e_{k}\right), n \geq 3$

é equivalente à base canônica de $c_{0}$, e assim, $c_{0} \hookrightarrow \mathcal{S}$ (ver [18], proposição 0.7 pg 2). Desta maneira, os espaços $\mathcal{S}$ e $\mathcal{S}^{\prime \prime}$ (bidual de $\mathcal{S}$ ) não satisfazem a condição $(\star \star)$, pois $c_{0}$ não é polinomialmente Schur. González e Gutiérrez em [22], proposição 2.4 demonstram que o 
espaço $\mathcal{S}$ tem a propriedade $(\mathbf{P})$.

Com argumento simples podemos observar que se um espaço de Banach $E$ é tal que para cada $m \in \mathbb{N}$, cada $P \in \mathcal{P}\left({ }^{m} E\right)$ é fracamente seqüencialmente contínuo na origem então $E$ tem a propriedade $(\mathbf{P})$ (ver [4]). Com este fato temos mais alguns exemplos de espaços que possuem a propriedade $(\mathbf{P})$.

Exemplos 2.2.7. 1. Se $w \notin l_{N} \forall N \in \mathbb{N}$, temos que $d_{*}(w ; 1)$ tem a propriedade $(\mathbf{P})$ (segue da proposição 2.4 de [37]).

2. O dual do espaço de Schreier, que denotamos por $\mathcal{S}^{\prime}$, tem a propriedade $(\mathbf{P})$ (segue do teorema 3.2 de [15]).

Para $\left(a_{n}\right)_{n} \in c_{00}$, seja

$$
\left\|\left(a_{n}\right)_{n}\right\|_{T_{J}^{*}}=\sup _{p_{1}<p_{2}<\ldots<p_{2 k}}\left\|\sum_{j=1}^{k}\left(a_{p_{2 j-1}}-a_{p_{2 j}}\right) t_{j}\right\|_{T^{*}} .
$$

O completamento de $c_{00}$ com respeito a norma $\|\cdot\|_{T_{J}^{*}}$, que é denotado por $T_{J}^{*}$, é chamado de espaço de Tsirelson*-James, ver [6] pg 1016-1017.

A seqüência $\left(e_{n}\right)_{n}$ é uma base de Schauder contrátil para $T_{J}^{*}$ (ver [6], proposição 8 e corolário 12). Pela proposição 14 de [6] temos que $\mathcal{P}\left({ }^{m} T_{J}^{*}\right)=\mathcal{P}_{w s c}\left({ }^{m} T_{J}^{*}\right)$ para cada $m \in \mathbb{N}$. Desta maneira, o espaço $T_{J}^{*}$ tem a propriedade $(\mathbf{P})$.

No que segue, temos por objetivo, mostrar que os espaços $T_{J}^{*}$ e $\left(T_{J}^{*}\right)^{\prime \prime}$ (bidual de $T_{J}^{*}$ ) não satisfazem a propriedade $(\star \star)$. Para isso, precisamos da seguinte propriedade:

Proposição 2.2.8. Seja E um espaço de Banach de dimensão infinita tal que $l_{1} \nrightarrow$ E e E é polinomialmente Schur. Então E admite polinômios que não são fracamente seqüencialmente contínuos.

Demonstração: Sendo $E$ um espaço de dimensão infinita, pelo lema 1.4.22 de [30] existe uma seqüência $\left(x_{n}\right)_{n} \subset E$ tal que $\left\|x_{n}\right\|=1$ e $\left\|x_{m}-x_{n}\right\| \geq 1$ sempre que $m \neq n$. Como 
por hipótese, $l_{1} \nrightarrow E$, então pelo teorema de Rosenthal-Dor, a seqüência $\left(x_{n}\right)_{n}$ admite uma subseqüência $\left(x_{n_{k}}\right)_{k}$ fracamente de Cauchy. Seja $\left(z_{k}\right)_{k}$ dada por $z_{k}=x_{n_{k+1}}-x_{n_{k}}$. Logo $z_{k} \stackrel{\omega}{\longrightarrow} 0$. Suponhamos, por absurdo, que $\mathcal{P}\left({ }^{m} E\right)=\mathcal{P}_{w s c}\left({ }^{m} E\right)$ para cada $m \in \mathbb{N}$. Então $P\left(z_{k}\right) \stackrel{k \rightarrow \infty}{\longrightarrow} 0$ para cada $P \in \mathcal{P}\left({ }^{m} E\right)$. Por hipótese, $E$ é polinomialmente Schur, e então, $\left\|z_{k}\right\| \stackrel{k \rightarrow \infty}{\longrightarrow} 0$. Uma contradição. Portanto, o espaço $E$ admite polinômios que não são fracamente seqüencialmente contínuos.

Corolário 2.2.9. Os espaços $T_{J}^{*} e\left(T_{J}^{*}\right)^{\prime \prime}$ não satisfazem a condição $(\star \star)$.

Demonstração: Pela proposição 14 de [6] temos que $\mathcal{P}\left({ }^{m} T_{J}^{*}\right)=\mathcal{P}_{w s c}\left({ }^{m} T_{J}^{*}\right)$ para cada $m \in \mathbb{N}$. Como $T_{J}^{*}$ admite base contrátil, pelo teorema 4.4.20 de [30] temos que $l_{1} \nrightarrow T_{J}^{*}$. Agora, pela proposição 2.2.8 temos que $T_{J}^{*}$ não é polinomialmente Schur. Desta maneira, pelo teorema 2.2.4 temos que o espaço $T_{J}^{*}$ não satisfaz a condição $(\star \star)$. E conseqüentemente, $\left(T_{J}^{*}\right)^{\prime \prime}$ não satisfaz a condição $(\star \star)$.

Observamos também, pela proposição 2.2.8, para uma seqüência de reais decrescente $w=\left(w_{i}\right) \in c_{0} \backslash l_{n} \forall n \in \mathbb{N}$ com $w_{1}=1$, o espaço $d_{*}(w ; 1)$ não é polinomialmente Schur.

Em [4], Aron, Choi e Llavona demonstram que todo espaço de Banach super-reflexivo satisfaz a condição $(\star \star)$ e tem a propriedade $(\mathbf{P})$. Assim, os espaços $l_{p}$ e $L_{p}[0,1]$ para $1<p<\infty$ têm a propriedade $(\mathbf{P})$. O espaço $d(w ; p), 1<p<\infty$, que satisfaz $\inf _{n} \frac{S(2 n)}{S(n)}>$ 1 onde $S(n)=\sum_{i=1}^{n} w_{i}$ também é super-reflexivo, e assim, pela proposição 2.2 .8 admite polinômios que não são fracamente seqüencialmente contínuos.

Um outro objetivo em nosso estudo era responder a seguinte pergunta, colocada por R. Alencar.

Todo espaço reflexivo tem a propriedade $(\mathbf{P})$ ?

Até o presente momento, não sabemos a resposta de tal questão. Com o objetivo de determinar exemplos de espaços reflexivos que não têm a propriedade $(\mathbf{P})$ passamos a estudar o espaço de Baernstein $\mathbb{B}_{\mathbf{p}}, 1<p<\infty$, reflexivo e não super-reflexivo, na tentativa de responder a pergunta de maneira negativa, mas conseguimos obter resultados positivos 
em relação à propriedade $(\mathbf{P})$.

No que segue vamos mostrar que o espaço de Baernstein $\mathbb{B}_{\mathbf{p}}, 1<p<\infty$, é polinomialmente Schur, satisfaz a condição $(\star \star)$ e tem a propriedade $(\mathbf{P})$. Para isso, precisamos do seguinte lema, cuja técnica de demonstração é análoga ao teorema de [36] no caso $p=2$.

Dados $x=\sum_{j=1}^{\infty} x_{j} e_{j} \in \mathbb{B}_{\mathbf{p}}$ e $F \subset \mathbb{N}$ finito, definamos: $x_{F}=\sum_{j \in F} x_{j} e_{j}$. Sendo $F$ finito, existe uma seqüência finita de subconjuntos de $F,\left\{\beta_{k}\right\}_{k=1}^{M} \operatorname{com} \beta_{k} \preceq \beta_{k+1}$ e $\left|\beta_{k}\right| \leq \min \beta_{k}$ tal que $\left\|x_{F}\right\|=\left(\sum_{k=1}^{M}\left(\sum_{j \in \beta_{k}}\left|x_{j}\right|\right)^{p}\right)^{\frac{1}{p}}$. E também vale $\left\|x_{F}\right\| \leq\|x\|$.

Lema 2.2.10. Seja $\left(y_{m}^{*}\right)_{m}$ uma seqüência limitada de blocos de $\left(e_{n}^{*}\right)_{n}$ em $\mathbb{B}_{\mathbf{p}}^{\prime}$ (dual de $\left.\mathbb{B}_{\mathbf{p}}\right)$. Então existe $C>0$ tal que $\left\|\sum_{m=1}^{N} y_{m}^{*}\right\| \leq C N^{\frac{1}{q}}$ para cada $N \in \mathbb{N} \operatorname{com} \frac{1}{p}+\frac{1}{q}=1$.

Demonstração: Seja $N \in \mathbb{N}$. Tomemos $C=\sup _{m}\left\|y_{m}^{*}\right\|$. Sendo $\left(y_{m}^{*}\right)_{m}$ uma seqüência de blocos, existem $\left(p_{m}\right)_{m}$ uma seqüência estritamente crescente de naturais e $\left(t_{m}\right)_{m}$ uma seqüência de escalares tais que $y_{m}^{*}=\sum_{j=p_{m}+1}^{p_{m+1}} t_{j} e_{j}^{*}$.

Para cada $m \in \mathbb{N}$, tomemos $F_{m}=\left\{p_{m}+1, \ldots, p_{m+1}\right\}$, e assim, $y_{m}^{*}=\sum_{j \in F_{m}} t_{j} e_{j}^{*}$.

Para cada $x \in \mathbb{B}_{\mathbf{p}}$ temos que $\left|\sum_{m=1}^{N} y_{m}^{*}(x)\right|=\left|\sum_{m=1}^{N} y_{m}^{*}\left(x_{F_{m}}\right)\right| \leq \sum_{m=1}^{N}\left\|y_{m}^{*}\right\|\left\|x_{F_{m}}\right\|$.

Assim, $\left\|\sum_{m=1}^{N} y_{m}^{*}\right\|=\sup _{\|x\|=1}\left|\left(\sum_{m=1}^{N} y_{m}^{*}\right)(x)\right| \leq C \sup _{\|x\| \leq 1} \sum_{m=1}^{N}\left\|x_{F_{m}}\right\|$.

Afirmação: Para cada $x \in \mathbb{B}_{\mathbf{p}}$ com $\|x\| \leq 1$ temos que $\sum_{m=1}^{N}\left\|x_{F_{m}}\right\|^{p} \leq 1$.

De fato: Para cada $m \in\{1, \ldots, N\}$ fixo existe uma seqüência finita de subconjuntos de $F_{m},\left\{\beta_{k}^{m}\right\}_{k=1}^{M_{m}} \operatorname{com} \beta_{k}^{m} \preceq \beta_{k+1}^{m}$ e $\left|\beta_{k}^{m}\right| \leq \min \beta_{k}^{m}$ tais que $\left\|x_{F_{m}}\right\|^{p}=\sum_{k=1}^{M_{m}}\left(\sum_{j \in \beta_{k}^{m}}\left|x_{j}\right|\right)^{p}$. Assim, $\sum_{m=1}^{N}\left\|x_{F_{m}}\right\|^{p}=\sum_{m=1}^{N}\left(\sum_{k=1}^{M_{m}}\left(\sum_{j \in \beta_{k}^{m}}\left|x_{j}\right|\right)^{p}\right)$. Sendo $F_{m} \preceq F_{m+1}$, por definição de $\|\cdot\|$, segue que $\sum_{m=1}^{N}\left\|x_{F_{m}}\right\|^{p} \leq\|x\|$, e assim, $\sum_{m=1}^{N}\left\|x_{F_{m}}\right\|^{p} \leq 1$.

Agora aplicando a desigualdade de Hölder, segue que

$$
\sum_{m=1}^{N}\left\|x_{F_{m}}\right\| \leq\left(\sum_{m=1}^{N}\left\|x_{F_{m}}\right\|^{p}\right)^{\frac{1}{p}}\left(\sum_{m=1}^{N} 1\right)^{\frac{1}{q}} \leq N^{\frac{1}{q}}
$$


Portanto, temos que $\left\|\sum_{m=1}^{N} y_{m}^{*}\right\| \leq C N^{\frac{1}{q}}$

Na próxima proposição faremos uso da noção de seqüência fracamente $q$-convergente. Seja $E$ um espaço de Banach e $\left(x_{n}\right)_{n}$ uma seqüência em $E$.

- Dizemos que $\left(x_{n}\right)_{n}$ é fracamente $q$-somável $(1<q<\infty)$ se $\sum_{n=1}^{\infty}\left|\phi\left(x_{n}\right)\right|^{q}<\infty$ para cada $\phi \in E^{\prime}$. Equivalentemente, existe uma constante $C>0$ tal que $\left\|\sum_{n=1}^{N} \alpha_{n} x_{n}\right\| \leq$ $C\left\|\left(\alpha_{n}\right)_{n}\right\|_{r} \forall N \in \mathbb{N}$ e para toda seqüência de escalares $\left(\alpha_{n}\right)_{n} \in l_{r} \operatorname{com} \frac{1}{r}+\frac{1}{q}=1$ (ver [24], pg 86-87).

- Dizemos que $\left(x_{n}\right)_{n}$ é fracamente $q$-convergente se existe $x \in E$ tal que a seqüência $\left(x_{n}-x\right)_{n}$ é fracamente $q$-somável.

Nos espaços $T^{*},\left(\bigoplus_{n=1}^{\infty} l_{1}^{(n)}\right)_{p}$ e $d(w ; p)$ para $1<p<\infty$ cada seqüência limitada admite subseqüência fracamente $q$-convergente para algum $q([16,17,23]$, proposição 3, pg 258 e proposição 2.1 respectivamente).

Proposição 2.2.11. O espaço de Baernstein $\mathbb{B}_{\mathbf{p}}, 1<p<\infty$, satisfaz a condição ( $\left.\star \star\right)$. E conseqüentemente, é polinomialmente Schur e tem a propriedade $\mathbf{P}$.

Demonstração: Pelo teorema 1.6 de [4] basta mostrar que cada seqüência limitada do seu dual admite uma subseqüência fracamente $q$-convergente para algum $q>1$.

Seja $\left(x_{n}^{*}\right)_{n}$ uma seqüência limitada em $\mathbb{B}_{\mathbf{p}}^{\prime}$. Sendo $\mathbb{B}_{\mathbf{p}}{ }^{\prime}$ um espaço reflexivo, $\left(x_{n}^{*}\right)_{n}$ admite uma subseqüência fracamente convergente, que vamos denotar por $\left(x_{n}^{*}\right)_{n}$. Seja $x^{*} \in \mathbb{B}_{\mathbf{p}}^{\prime}$ tal que $x_{n}^{*} \stackrel{\omega}{\longrightarrow} x^{*}$. Para cada $n \in \mathbb{N}$, seja $y_{n}^{*}=x_{n}^{*}-x^{*}$. Sem perda de generalidade, podemos supor que $\inf _{n}\left\|y_{n}^{*}\right\|>0$. Pelo Princípio de Seleção de Bessaga-Pelczyński (ver teorema 4.3.19 em [30]), $\left(y_{n}^{*}\right)_{n}$ admite uma subseqüência, que vamos denotar por $\left(y_{n_{m}}^{*}\right)_{m}$, que é equivalente a uma seqüência de bases de blocos com respeito a $\left(e_{j}^{*}\right)_{j}$. Pelo lema 2.2.10, existe $C>0$ tal que $\left\|\sum_{m=1}^{N} y_{n_{m}}^{*}\right\| \leq C N^{\frac{1}{r}}$ para cada $N \in \mathbb{N} \operatorname{com} \frac{1}{p}+\frac{1}{r}=1$. Agora, pelo teorema 2.3 de [17], $\left(y_{n_{m}}^{*}\right)_{m}$ é fracamente $q$-convergente com $\frac{1}{q}+\frac{1}{s}=1$ para todo 
$s>p$. E assim, a subseqüência $\left(x_{n_{m}}^{*}\right)_{m}$ é fracamente $q$-convergente para todo $q$ com $\frac{1}{q}+\frac{1}{s}=1$ e $s>p$. Portanto, pelo teorema 1.6 de [4], o espaço $\mathbb{B}_{\mathbf{p}}$ satisfaz a condição $(\star \star)$. E conseqüentemente, pelo teorema 2.2.4, o espaço $\mathbb{B}_{\mathbf{p}}$ tem a propriedade $(\mathbf{P})$ e é polinomialmente Schur.

Desta maneira, podemos observar que, pela proposição 2.2.8, o espaço de Baernstein admite polinômios que não são fracamente seqüencialmente contínuos. Também, pelo teorema 1.6 de [4] observamos que os espaços $T$ (dual de $\left.T^{*}\right),\left(\bigoplus_{n=1}^{\infty} l_{\infty}^{(n)}\right)_{p}$ e $d_{*}(w ; p)$ com $1<p<\infty$, satisfazem a condição $(\star \star)$, são polinomialmente Schur e têm a propriedade $(\mathbf{P})$, e assim, pela proposição 2.2 .8 admitem polinômios que não são fracamente seqüêncialmente contínuos.

Em [4], Aron, Choi e Llavona questionam o seguinte fato: Existe um espaço de Banach que não satisfaz a propriedade $(\mathbf{P})$ ?

Inicialmente, Choi e Kim em [19] demonstram que se o espaço $E$ for polinomialmente Schur e $\mathcal{P}\left({ }^{n} E\right)$ for separável para cada $n \in \mathbb{N}$ então o espaço $E$ não tem a propriedade (P). Porém, não se conhece exemplos de espaços que satisfaçam tais condições. Por outro lado, se $E$ é algum dos espaços: $\left(\bigoplus_{n=1}^{\infty} l_{2}^{(n)}\right)_{0}, \mathbb{B}_{\mathbf{p}},\left(\bigoplus_{n=1}^{\infty} l_{\infty}^{(n)}\right)_{p}, d_{*}(w ; p), 1<p<\infty, T$ tem-se que o espaço $\mathcal{P}(E)$ não é separável. Em 1999 Castillo, García e Gonzalo [15] apresentam um exemplo de espaço de Banach que não satisfaz a propriedade (P). Vamos utilizar a técnica apresentada por eles para demonstrar a proposição 2.2.12.

Vamos denotar por $\left(E \oplus E^{\prime}\right)_{p}$ o espaço de Banach formado pelos pares $(x, f)$ com $x \in E$ e $f \in E^{\prime}$ munido da norma $\|(x, f)\|_{p}=\left(\|x\|^{p}+\|f\|^{p}\right)^{\frac{1}{p}}$ se $p>0$ e $\|(x, f)\|_{0}=\max \{\|x\|,\|f\|\}$.

Proposição 2.2.12. Seja $E$ um espaço de Banach tal que $E$ não tem a propriedade de Dunford-Pettis. Suponhamos que $\mathcal{P}\left({ }^{m} E\right)=\mathcal{P}_{\text {wsc }}\left({ }^{m} E\right)$ e $\mathcal{P}\left({ }^{m} E^{\prime}\right)=\mathcal{P}_{\text {wsc }}\left({ }^{m} E^{\prime}\right)$ para cada $m \in \mathbb{N}$. Então o espaço $\left(E \oplus E^{\prime}\right)_{p}$ não tem a propriedade $(\mathbf{P})$ para cada $p \geq 0$. 
Demonstração: Primeiramente vamos demonstrar que $\left(E \oplus E^{\prime}\right)_{p}$ não é isomorfo nem a $E$ e nem a $E^{\prime}$. Suponhamos por absurdo que $\left(E \oplus E^{\prime}\right)_{p}$ seja isomorfo a $E$. Como $\mathcal{P}\left({ }^{m} E\right)=\mathcal{P}_{w s c}\left({ }^{m} E\right)$ temos que $\mathcal{P}\left(m\left(E \oplus E^{\prime}\right)_{p}\right)=\mathcal{P}_{w s c}\left(m\left(E \oplus E^{\prime}\right)_{p}\right)$ para cada $m \in \mathbb{N}$. Por hipótese, $E$ não tem a propriedade de Dunford-Pettis, e então existem seqüências $\left(x_{n}\right)_{n} \subset E$ e $\left(x_{n}^{\star}\right)_{n} \subset E^{\prime}$ tais que $x_{n} \stackrel{\omega}{\longrightarrow} 0$ em $E$ e $x_{n}^{\star} \stackrel{\omega}{\longrightarrow} 0$ em $E^{\prime}$ mas $x_{n}^{\star}\left(x_{n}\right)^{n \rightarrow \infty} \nrightarrow 0$. Assim, existe $\epsilon>0$ e existe uma seqüência de naturais $\left(n_{j}\right)_{j}$ tal que $\left|x_{n_{j}}^{\star}\left(x_{n_{j}}\right)\right| \geq \epsilon$. Agora, definamos $P:\left(E \oplus E^{\prime}\right)_{p} \longrightarrow \mathbb{K}$ por $P(x, f)=f(x)$ para cada $(x, f) \in\left(E \oplus E^{\prime}\right)_{p}$. Então $P \in \mathcal{P}\left(2\left(E \oplus E^{\prime}\right)_{p}\right) \backslash \mathcal{P}_{w s c}\left(2\left(E \oplus E^{\prime}\right)_{p}\right)$ pois $\left|P\left(x_{n_{j}}, x_{n_{j}}^{\star}\right)\right|=\left|x_{n_{j}}^{\star}\left(x_{n_{j}}\right)\right| \geq \epsilon$ para cada $j \in \mathbb{N}$ e $\left(x_{n_{j}}, x_{n_{j}}^{\prime}\right) \stackrel{\omega}{\longrightarrow}(0,0)$. Uma contradição. E assim, $\left(E \oplus E^{\prime}\right)_{p}$ não é isomorfo a $E$. Do fato de $E$ não ter a propriedade de Dunford-Pettis segue que $E^{\prime}$ não tem a propriedade de Dunford-Pettis. E de maneira análoga provamos que $\left(E \oplus E^{\prime}\right)_{p}$ não é isomorfo a $E^{\prime}$. Agora vamos provar que $\left(E \oplus E^{\prime}\right)_{p}$ não tem a propriedade $(\mathbf{P})$. Por hipótese, existem seqüências $\left(x_{n}\right)_{n} \subset E,\left(x_{n}^{*}\right)_{n} \subset E^{\prime}$ tais que $x_{n} \stackrel{\omega}{\longrightarrow} 0$ em $E$ e $x_{n}^{*} \stackrel{\omega}{\longrightarrow} 0$ em $E^{\prime}$ e existe uma seqüência de naturais $\left(n_{j}\right)_{j}$ tal que $\left|x_{n_{j}}^{*}\left(x_{n_{j}}\right)\right| \geq 1$.

Para cada $m \in \mathbb{N}$ e $Q \in \mathcal{P}\left(m\left(E \oplus E^{\prime}\right)_{p}\right)$ temos que $Q\left(\left(x_{n_{j}}, 0\right)-Q\left(\left(0, x_{n_{j}}^{*}\right)\right) \stackrel{j \rightarrow \infty}{\longrightarrow} 0\right.$, pois o polinômio $Q$ restrito aos subespaços $E$ e $E^{\prime}$ é fracamente seqüencialmente contínuo.

Tomemos $P:\left(E \oplus E^{\prime}\right)_{p} \longrightarrow \mathbb{K}$ dado por $P(x, f)=f(x)$ para cada $(x, f) \in\left(E \oplus E^{\prime}\right)_{p}$. Temos que $P \in \mathcal{P}\left(2\left(E \oplus E^{\prime}\right)_{p}\right)$ e $\left|P\left(\left(x_{n_{j}}, 0\right)-\left(0, x_{n_{j}}^{*}\right)\right)\right|=\left|x_{n_{j}}^{*}\left(x_{n_{j}}\right)\right| \geq 1$ para cada $j \in \mathbb{N}$. Portanto $\left(E \oplus E^{\prime}\right)_{p}$ não tem a propriedade $(\mathbf{P})$.

Observamos que a propriedade $(\mathbf{P})$ é preservada por isomorfismo, e assim, $\left(E^{\prime} \oplus E\right)_{p}$ não tem a propriedade $(\mathbf{P})$.

Um exemplo de espaço que satisfaz as condições da proposição 2.2.12 é o espaço apresentado em [15].

Exemplo 2.2.13. Seja $w=\left(w_{i}\right)_{i} \in c_{0} \backslash l_{1}$ uma seqüência decrescente de reais com $w_{1}=1$ 
e para cada $p \in \mathbb{N}$, temos

$$
0=\lim _{N \rightarrow \infty} \inf \frac{\sum_{i=1}^{N} w_{i}}{N^{\frac{1}{p}}} \leq \lim _{N \rightarrow \infty} \sup \frac{\sum_{i=1}^{N} w_{i}}{N^{\frac{1}{p}}}=\infty .
$$

Os espaços $\left(d_{*}(w ; 1) \oplus d(w ; 1)\right)_{q} e\left(d(w ; 1) \oplus d_{*}(w ; 1)\right)_{q}$ não possuem a propriedade $\mathbf{( P )}$ para cada $q \geq 0$.

De fato: Para qualquer seqüencia decrescente de reais $w=\left(w_{i}\right)_{i} \in c_{0} \backslash l_{1}$ com $w_{1}=1$, o espaço $d_{*}(w ; 1)$ não tem a propriedade de Dunford-Pettis (pois $e_{n} \stackrel{\omega}{\longrightarrow} 0$ e $e_{n}^{\prime} \stackrel{\omega}{\longrightarrow} 0$ ). Se a seqüência $w=\left(w_{i}\right)_{i}$ satisfaz a desigualdade 2.1, pelo teorema 5.4 de [15] temos que $\mathcal{P}\left({ }^{m} d_{*}(w ; 1)\right)=\mathcal{P}_{w s c}\left({ }^{m} d_{*}(w ; 1)\right)$ e $\mathcal{P}\left({ }^{m} d(w ; 1)\right)=\mathcal{P}_{w s c}\left({ }^{m} d(w ; 1)\right)$ para cada $m \in \mathbb{N}$.

Corolário 2.2.14. Seja E um espaço de dimensão infinita tal que $l_{1} \hookrightarrow E^{\prime}$. Suponhamos que $\mathcal{P}\left({ }^{m} E\right)=\mathcal{P}_{\text {wsc }}\left({ }^{m} E\right)$ e $\mathcal{P}\left({ }^{m} E^{\prime}\right)=\mathcal{P}_{\text {wsc }}\left({ }^{m} E^{\prime}\right)$ para cada $m \in \mathbb{N}$. Então o espaço $\left(E \oplus E^{\prime}\right)_{p}$ não tem a propriedade $\mathbf{( P )}$ para cada $p \geq 0$.

Demonstração: Sendo $E^{\prime}$ um espaço de dimensão infinita, pelo lema 1.4.22 de [30] existe uma seqüência $\left(x_{n}\right)_{n} \subset E^{\prime}$ tal que $\left\|x_{n}\right\|=1$ e $\left\|x_{m}-x_{n}\right\| \geq 1$ sempre que $m \neq n$. Como por hipótese, $l_{1} \hookrightarrow E^{\prime}$, então pelo teorema de Rosenthal-Dor, a seqüência $\left(x_{n}\right)_{n}$ admite uma subseqüência $\left(x_{n_{k}}\right)_{k}$ fracamente de Cauchy. Para a seqüência $\left(z_{k}\right)_{k}$ dada por $z_{k}=x_{n_{k+1}}-x_{n_{k}}$ temos que $z_{k} \stackrel{\omega}{\longrightarrow} 0$ e $\left\|z_{k}\right\| \geq 1 \forall k \in \mathbb{N}$. Ou seja, $E^{\prime}$ não é de Schur. Novamente, pelo fato de $l_{1} \hookrightarrow E^{\prime}$, pelo corolário 10 de [32] temos que $l_{1} \nrightarrow E$. E assim, pela proposição 1.0.6(2) E não tem a propriedade de Dunford-Pettis. E agora, pela proposição 2.2 .12 temos que o espaço $\left(E \oplus E^{\prime}\right)_{p}$ não tem a propriedade $(\mathbf{P}), p \in \mathbb{N}_{0}$.

Até o presente momento não sabemos se existem espaços de Banach de dimensão infinita que satisfaçam as condições do corolário 2.2.14.

No que segue vamos apresentar mais alguns exemplos de espaços de Banach que não satisfazem a condição $(\star \star)$.

Dado um espaço de Banach $E$, vamos denotar por $\mathcal{H}(E)$ o espaço de todas as funções holomorfas definidas sobre $E$. E por $\mathcal{H}_{b}(E)$ o subespaço de todas as funções $f \in \mathcal{H}(E)$ 
tais que $f$ é limitada sobre cada subconjunto limitado de $E$, e tal espaço é chamado de espaço das funções inteiras do tipo limitada.

Definição 2.2.15. Seja E um espaço de Banach.

a) Dizemos que uma seqüência $\left(x_{n}\right)_{n}$ de E é holomorficamente convergente para algum $x \in E$ se para cada $f \in \mathcal{H}(E)$ a condição $\lim _{n \rightarrow \infty} f\left(x_{n}\right)=f(x)$ é satisfeita.

b) Se para cada seqüência $\left(x_{n}\right)_{n}$ de E holomorficamente convergente a $0 \in E$ tem-se que $\left\|x_{n}\right\| \stackrel{n \rightarrow \infty}{\longrightarrow} 0$, dizemos que o espaço é holomorficamente Schur.

A noção de seqüência holomorficamente convergente foi introduzida e estudada por Petunin e Savnik em [33]. De imediato vemos que todo espaço polinomialmente Schur é holomorficamente Schur. A recíproca é falsa, pois todo espaço separável é holomorficamente Schur, ver teorema $3 \mathrm{em}$ [33]. O espaço $l_{\infty}$ não é holomorficamente Schur, demonstrado por Aron, Choi e Llavona em [4] (exemplo 1.7). A seguir, vamos mostrar que o espaço $\left(\bigoplus_{n=1}^{\infty} l_{2}^{(n)}\right)_{\infty}$ não é holomorficamente Schur.

Definição 2.2.16. Dizemos que um subconjunto $A$ de um espaço de Banach $E$ é limitante se para cada $f \in \mathcal{H}(E)$, f é limitada sobre $A$.

A noção de conjunto limitante está relacionada a extensão de funções holomorfas de um subespaço de Banach $E$ a $F$, isto é, um dado conjunto limitado de $E$ é limitante em $F$ se e somente se cada $f \in \mathcal{H}(E)$ que tem extensão holomorfa para $F$ é de tipo limitada. Em particular, cada subconjunto limitado de um espaço de Banach $E$ é limitante em $E^{\prime \prime}$ se e somente se cada $f \in \mathcal{H}(E)$ que admite uma extensão holomorfa para $E^{\prime \prime}$ é uma função de tipo limitada.

Em um trabalho recente, Carrión, Galindo e Lourenço [14] demonstram que cada subconjunto limitado de $\left(\bigoplus_{n=1}^{\infty} l_{2}^{(n)}\right)_{0}$, predual do espaço de Stegall, é limitante em $\left(\bigoplus_{n=1}^{\infty} l_{2}^{(n)}\right)_{\infty}$, ver teorema 2.4 em [14]. Desta maneira, podemos demonstrar:

Proposição 2.2.17. O espaço $\left(\bigoplus_{n=1}^{\infty} l_{2}^{(n)}\right)_{\infty}$ não é holomorficamente Schur. 
Demonstração: Para simplificar a notação, vamos denotar por $E=\left(\bigoplus_{n=1}^{\infty} l_{2}^{(n)}\right)_{0}$ e $E^{\prime \prime}=\left(\bigoplus_{n=1}^{\infty} l_{2}^{(n)}\right)_{\infty}$. Basta mostrar que existe uma seqüência $\left(x_{n}\right)_{n}$ em $E^{\prime \prime}$ tal que $f\left(x_{n}\right) \stackrel{n \rightarrow \infty}{\longrightarrow} f(0) \forall f \in \mathcal{H}\left(E^{\prime \prime}\right)$ e $\left\|x_{n}\right\| \not \rightarrow 0$. Sejam $\left(x_{n}\right)_{n}$ a base canônica de $E$ e $f \in \mathcal{H}\left(E^{\prime \prime}\right)$, tal que $f=\sum_{j=0}^{\infty} P_{j}$, onde $\sum_{j=0}^{\infty} P_{j}$ representa a série de Taylor de $f$ na origem. Seja $g=\left.f\right|_{E}$, pelo teorema 2.4 de [14], cada subconjunto limitado de $E$ é limitante em $E^{\prime \prime}$, temos que a função $g$ é do tipo limitada, ou seja, $g \in \mathcal{H}_{b}(E)$. Em particular, para $B_{E}$ (bola unitária fechada de $E$ ), $g$ é o limite uniforme de $\sum_{j=0}^{\infty} P_{j}$. Desta maneira, dado $\epsilon>0$ existe $m_{0} \in \mathbb{N}$ tal que $\left|g(x)-\sum_{j=0}^{m_{0}} P_{j}(x)\right|<\frac{\epsilon}{2}$ para todo $x \in B_{E}$. Para cada $n \in \mathbb{N}$, temos que $\left|f\left(e_{n}\right)-f(0)\right|=\left|g\left(e_{n}\right)-g(0)\right| \leq$

$$
\left|g\left(e_{n}\right)-\sum_{j=0}^{m_{0}} P_{j}\left(e_{n}\right)\right|+\left|\sum_{j=0}^{m_{0}} P_{j}\left(e_{n}\right)-P_{0}(0)\right|=\mid g\left(e_{n}-\sum_{j=1}^{m_{0}} P_{j}\left(e_{n}\right) \mid .\right.
$$

Sendo $E$ um espaço que tem a propriedade de Dunford-Pettis, segue que $P_{j}\left(e_{n}\right) \stackrel{n \rightarrow \infty}{\longrightarrow} 0$ para cada $j=1, \ldots, m_{0}$ pois $e_{n} \stackrel{\omega}{\longrightarrow} 0$. Assim, existe $n_{0} \in \mathbb{N}$ tal que $\left|P_{j}\left(e_{n}\right)\right|<\frac{\epsilon}{2 m_{0}} \forall n \geq n_{0} \mathrm{e}$ cada $j=1, \ldots, m_{0}$. Desta maneira, $\left|f\left(e_{n}\right)-f(0)\right|<\epsilon \forall n \geq n_{0}$, ou seja, $f\left(e_{n}\right) \stackrel{n \rightarrow \infty}{\longrightarrow} f(0)$. Portanto, o espaço $\left(\bigoplus_{n=1}^{\infty} l_{2}^{(n)}\right)_{\infty}$ não é holomorficamente Schur.

Stegall em [38] afirma que $E=\left(\bigoplus_{n=1}^{\infty} l_{2}^{(n)}\right)_{1}$ é um espaço de Schur e daí tem a propriedade de Diunford-Pettis e o seu dual $E^{\prime}=\left(\bigoplus_{n=1}^{\infty} l_{2}^{(n)}\right)_{\infty}$ não tem a propriedade de Dunford-Pettis.

Assim, segue do teorema 2.2.4 que o espaço de Stegall $\left(\bigoplus_{n=1}^{\infty} l_{2}^{(n)}\right)_{1}$ satisfaz a condição $(\star \star)$ e tem a propriedade $(\mathbf{P})$. O seu predual $\left(\bigoplus_{n=1}^{\infty} l_{2}^{(n)}\right)_{0}$ não satisfaz a condição $(\star \star)$ e tem a propriedade $(\mathbf{P})$. E o seu dual $\left(\bigoplus_{n=1}^{\infty} l_{2}^{(n)}\right)_{\infty}$ não satisfaz a condição $(\star \star)$.

Observação 2.2.18. Com argumentações simples podemos observar que:

i) A propriedade holomorficamente Schur é preservada por isomorfismos. 
ii) Seja E um espaço de Banach holomorficamente Schur. Então cada subespaço fechado de E também é holomorficamente Schur.

Desta maneira, conseguimos exibir mais exemplos de espaços de Banach que não são holomorficamente Schur.

Exemplos 2.2.19. Os espaços $L_{\infty}[0,1], \mathcal{L}\left(l_{2}, l_{2}\right), \hat{\bigotimes}_{n, \pi} l_{\infty}, d^{*}(w ; 1), \mathcal{S}^{\prime \prime}, \mathcal{L}\left({ }^{n} l_{p}\right)$ e $\mathcal{L}\left({ }^{n} L_{q}[0,1]\right)$ com $n \geq 2,1 \leq p \leq 2$ e $1<q<2$ não são holomorficamente Schur, e conseqüentemente, não satisfazem a condição $(\star \star)$.

De fato: Temos que $L_{\infty}[0,1] \equiv l_{\infty}, l_{\infty} \hookrightarrow \mathcal{L}\left(l_{2} ; l_{2}\right)$ e $\hat{\bigotimes}_{n, \pi} l_{\infty}$ contém uma cópia complementada de $l_{\infty}$. O espaço $d(w ; 1)$ tem um subespaço complementado isomorfo a $l_{1}$, ver teorema 4.e.3 de [26]. Pelo teorema de Bessaga-Pelczyński, os espaços $d^{*}(w ; 1)$ e $\mathcal{S}^{\prime \prime}$ contém uma cópia complementada de $l_{\infty}$.

Sejam $n \in \mathbb{N}$ com $n \geq 2,1 \leq p \leq 2$ e $1<q<2$. No caso $n=2$, em [35] teorema 2.9 e exemplo 2.23 , é demonstrado que $\mathcal{L}\left({ }^{2} l_{p}\right)=\left(\underset{2, \pi}{\otimes} l_{p}\right)^{\prime}$ e $\hat{\bigotimes}_{2, \pi} l_{p}$ contém um subespaço complementado isomorfo a $l_{1}$. Com uma demonstração análoga, o resultado é válido para todo $n \in \mathbb{N}$ com $n \geq 2$, ou seja, $\mathcal{L}\left({ }^{n} l_{p}\right)=\left(\hat{\bigotimes} l_{n}\right)^{\prime}$ e o espaço $\bigotimes_{n, \pi} l_{p}$ contém um subespaço complementado isomorfo a $l_{1}$. Pelo teorema de Bessaga-Pelczyński, o espaço $\mathcal{L}\left({ }^{n} l_{p}\right)$ tem uma cópia complementada de $l_{\infty}$. De maneira análoga ao caso anterior, temos que $\mathcal{L}\left({ }^{n} L_{q}[0,1]\right)=\left(\hat{\otimes} L_{n, \pi}[0,1]\right)^{\prime}$. No corolário 2.26 de [35], é demonstrado que o espaço $\underset{2, \pi}{\otimes} L_{q}[0,1]$ contém um subespaço complementado isomorfo a $l_{1}$. Com demonstração análoga, tem-se que $\hat{\bigotimes}_{n, \pi} L_{q}[0,1]$ contém um subespaço complementado isomorfo a $l_{1}$. Agora, pelo teorema de Bessaga-Pelczyński, o espaço $\mathcal{L}\left({ }^{n} L_{q}[0,1]\right)$ tem uma cópia complementada de $l_{\infty}$.

\subsection{A propriedade (RP)}

Aron, Choi e Llavona em [4] definiram a propriedade (RP) como sendo a recíproca da propriedade $(\mathbf{P})$. 
Definição 2.3.1. Seja E um espaço de Banach. Dizemos que E tem a propriedade (RP) se para quaisquer duas seqüências $\left(u_{j}\right)_{j},\left(v_{j}\right)_{j}$ limitadas em $E$ tais que para cada $n \in \mathbb{N}$ e cada $P \in \mathcal{P}\left({ }^{n} E\right)$ com $\left|P\left(u_{j}-v_{j}\right)\right| \stackrel{j \rightarrow \infty}{\longrightarrow} 0$ tem-se que $\left|Q\left(u_{j}\right)-Q\left(v_{j}\right)\right| \stackrel{j \rightarrow \infty}{\longrightarrow} 0$ para cada $Q \in \mathcal{P}\left({ }^{m} E\right)$ e cada $m \in \mathbb{N}$.

Ou seja, um espaço de Banach $E$ tem a propriedade $(\mathbf{R P})$ se para cada $m \in \mathbb{N}$, cada $Q \in \mathcal{P}\left({ }^{m} E\right)$ e cada $W \subset E$ limitado, $\left.Q\right|_{W}$ é uniformemente seqüencialmente contínuo na topologia polinomial fraca.

O seguinte teorema foi apresentado em [4]:

Teorema 2.3.2 (Aron, Choi e Llavona, [4]). Seja E um espaço de Banach. Se E é polinomialmente Schur então E tem a propriedade (RP).

Assim, do teorema 2.3.2 segue que os espaços: $\left(\bigoplus_{n=1}^{\infty} l_{2 n}\right)_{2},\left(\bigoplus_{n=1}^{\infty} l_{2}^{(n)}\right)_{1}, \mathbb{B}_{\mathbf{p}},\left(\bigoplus_{n=1}^{\infty} l_{\infty}^{(n)}\right)_{p}$, $d_{*}(w ; p), 1<p<\infty$ e $T$ têm a propriedade $(\mathbf{R P})$. Como também o espaço $d(w ; p)$, $1<p<\infty$, sempre que $\inf _{n} \frac{S(2 n)}{S(n)}>1 \operatorname{com} S(n)=\sum_{i=1}^{n} w_{i}$.

Não temos a recíproca para o teorema 2.3.2. Os espaços $c_{0},\left(\bigoplus_{n=1}^{\infty} l_{2}^{(n)}\right)_{0}, T^{*}, T_{J}^{*} \mathrm{e}$ $d_{*}(w ; 1)$ com $w=\left(w_{i}\right)_{i} \in c_{0} \backslash l_{N} \forall N \in \mathbb{N}$ uma seqüência decrescente de reais com $w_{1}=1$ não são polinomialmente Schur e possuem a propriedade $(\mathbf{R P})$, pois para cada $m \in \mathbb{N}$ $\mathcal{P}\left({ }^{m} E\right)=\mathcal{P}_{w u}\left({ }^{m} E\right)$.

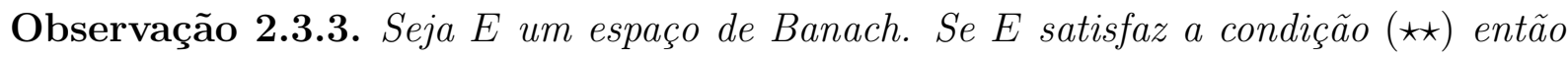
E tem a propriedade $(\mathbf{R P})$.

Pelo teorema 2.3.2 temos que o espaço de Schreier não é polinomialmente Schur pois González e Gutiérrez em [22], proposição 2.3 demonstram que o espaço $\mathcal{S}$ não tem a propriedade ( $\mathbf{R P})$.

Em [4], Aron, Choi e Llavona apresentam alguns exemplos de espaços que não têm a propriedade $(\mathbf{R P})$. Dentre eles, os espaços $C[0,1]$ e $L_{\infty}[0,1] \approx l_{\infty}$ não têm a propriedade (RP). No que segue, temos por objetivo mostrar que o dual de certos espaços de Lorentz não têm a propriedade (RP). Para isso, vamos utilizar o seguinte lema: 
Lema 2.3.4. Seja E um espaço de Banach que tem propriedade (RP). Então cada subespaço complementado também tem a propriedade $(\mathbf{R P})$.

Observamos que resultado análogo também vale para a propriedade $(\mathbf{P})$.

Proposição 2.3.5. Seja E um espaço de Banach.

1. Se $l_{\infty}$ é isomorfo a um subespaço complementado de E então E não tem a propriedade $(\mathbf{R P})$.

2. Suponhamos que E admite um subespaço complementado isomorfo ao espaço $l_{1}$. Então $E^{\prime}$ não tem a propriedade $(\mathbf{R P})$.

Demonstração: (1) Suponhamos que $E$ tenha a propriedade (RP). Então, pelo lema 2.3.4 temos que $l_{\infty}$ tem a propriedade $(\mathbf{R P})$. Uma contradição. Portanto $E$ não tem a propriedade $(\mathbf{R P})$.

(2) Seja $E$ um espaço tal que $l_{1}$ é isomorfo a um subespaço complementado de $E$. Pelo teorema de Bessaga-Pelczyński, $l_{\infty}$ é isomorfo a um subespaço complementado de $E^{\prime}$. Agora, por (1) temos que o espaço $E^{\prime}$ não tem a propriedade (RP).

Para cada $n \in \mathbb{N}$, o espaço $\hat{\bigotimes}_{n, \pi} E$ (o produto $n$-tensorial projetivo) contém uma cópia complementada de $E$. Assim, pela proposição 2.3.5 temos que o espaço $\hat{\bigotimes}_{n, \pi} l_{\infty}$ não tem a propriedade $(\mathbf{R P})$.

Exemplos 2.3.6. 1. Os espaços $d^{*}(w ; 1)$ e $\mathcal{S}^{\prime \prime}$ não têm a propriedade $\left.\mathbf{R P}\right)$.

2. Para $p, n \in \mathbb{N}, 1 \leq p \leq 2$ e $n \geq 2$, o espaço $\mathcal{L}\left({ }^{n} l_{p}\right)$ não tem a propriedade $(\mathbf{R P})$.

3. Para $p, n \in \mathbb{N}, 1<p<2$ e $n \geq 2$, o espaço $\mathcal{L}\left({ }^{n} L_{p}[0,1]\right)$ não tem a propriedade (RP).

Vamos finalizar a seção fazendo algumas observações sobre a propriedade (RP) no espaço $C(K)$, onde $K$ é compacto e Hausdorff. 
Aron, Choi e Llavona em [4] demonstram que se um espaço de Banach $E$ tem a propriedade de Dunford-Pettis e $l_{1} \hookrightarrow E$ então $E$ tem a propriedade (RP). Desta maneira, temos que:

Exemplos 2.3.7. Seja K um espaço compacto e Hausdorff.

1. Se $K$ é disperso então $C(K)$ tem a propriedade $(\mathbf{R P})$.

2. Se $K$ é métrico e não enumerável então $C(K)$ não tem a propriedade $(\mathbf{R P})$.

De fato: Suponhamos que $K$ é compacto, Hausdorff e disperso. É conhecido que $K$ é disperso se e somente se $l_{1} \nrightarrow C(K)$. Como $C(K)$ tem a propriedade de Dunford-Pettis segue que o espaço $C(K)$ tem a propriedade (RP).

Suponhamos que $K$ é compacto, métrico e não enumerável. Neste caso, $C(K)$ é isomorfo a $C([0,1])$, e como a propriedade $(\mathbf{R P})$ é preservada por isomorfismos, segue que $C(K)$ não tem a propriedade $\mathbf{R P}$. 


\section{Capítulo 3}

\section{Os conjuntos $C_{\tau}(P)$}

Dados $E, F$ espaços de Banach, $N \in \mathbb{N}$ e $P: E \longrightarrow F$ um polinômio $N$-homogêneo contínuo, neste capítulo temos por objetivo estudar o conjunto dos pontos $x \in E$ tais que $P$ é $\tau$ contínuo sobre os limitados de $x$ ou $\tau$-seqüencialmente contínuo em $x$, para determinadas topologias $\tau$ definidas em $E$. Mais especificamente, faremos um estudo quando $\tau$ é a topologia fraca, denotada por $\omega$. E quando o espaço $E$ for um espaço dual, também vamos analisar alguns resultados para a $\tau$ sendo a topologia fraca-estrela, denotada por $\omega^{\star}$.

Em [5], Aron e Dimant estudaram o conjunto de continuidade seqüencial fraca para os polinômios $P: E \longrightarrow \mathbb{K}$, obtendo algumas propriedades. Na seção 3.1 vamos estudar os polinômios contínuos $N$-homogêneos $P: E \longrightarrow F$ com $F \neq \mathbb{K}$ e $E$ munido da topologia $\omega$ e fazer algumas conexões com os resultados obtidos por Aron e Dimant em [5]. E na seção 3.2 vamos trabalhar com $E$ um espaço dual munido da topologia $\omega^{\star}$. 


\section{$3.1 \quad$ O conjunto $C(P)$}

Denotaremos por $\mathcal{P}_{w s c}\left({ }^{N} E ; F\right)$ o conjunto de todos os polinômios $P \in \mathcal{P}\left({ }^{N} E ; F\right)$ que são fracamente seqüencialmente contínuos em todos os pontos de $E$. Quando $F=\mathbb{K}$, vamos usar a notação usual $\mathcal{P}_{w s c}\left({ }^{N} E\right)$. E vamos denotar por $\mathcal{P}_{w s c(0)}\left({ }^{N} E ; F\right)$ o espaço formado pelos $P \in \mathcal{P}\left({ }^{N} E ; F\right)$ que são $\omega$-seqüencialmente contínuos em 0 .

Os espaços $\mathcal{P}_{w s c}\left({ }^{N} E\right)$ e $\mathcal{P}_{w s c(0)}\left({ }^{N} E\right)$ de modo geral não coincidem, como mostra o seguinte exemplo: Sejam $p \in] 1, \infty\left[\right.$ fixo e $\left\{N_{i}\right\}_{i}$ uma partição de $\mathbb{N}$ onde cada $N_{i}$ é um conjunto infinito. Tomemos $N \in \mathbb{N}$ o menor inteiro tal que $N \geq p$. Para o polinômio $P: l_{p} \longrightarrow \mathbb{K}$ definido por $P(x)=\sum_{i=1}^{\infty} \frac{x_{i}^{2}}{2^{i}} \sum_{j \in N_{i}} x_{j}^{N}$ para cada $x=\left(x_{i}\right)_{i} \in l_{p}$, temos que $P$ é fracamente seqüencialmente contínuo em 0 e não é nos demais pontos de $l_{p}$ (ver o exemplo 7(4) em [5]). Por outro lado, $\mathcal{P}_{w s c}\left({ }^{N} l_{p}\right)=\mathcal{P}\left({ }^{N} l_{p}\right) \forall N<p$ (é o conhecido Teorema de Pitt).

Agora para $E=c_{0}$, sabemos que $\mathcal{P}_{w s c}\left({ }^{N} c_{0}\right)=\mathcal{P}\left({ }^{N} c_{0}\right) \forall N \in \mathbb{N}$, uma vez que $c_{0}$ tem a propriedade de Dunford-Pettis.

No que segue vamos exibir exemplos de polinômios nos espaços de Banach acima, para salientar que o espaço $\mathcal{P}_{w s c}\left({ }^{N} E ; F\right)$, ou seja, os polinômios fracamente seqüencialmente contínuos com valores vetoriais, tem comportamentos totalmente distintos.

Exemplos 3.1.1. a) Sejam $p \in] 1, \infty\left[\right.$ e $N \in \mathbb{N}$. O polinômio $P: l_{p} \longrightarrow l_{p}$ definido por $P(x)=\sum_{n=1}^{\infty} x_{n}^{N} e_{n}$ para cada $x=\left(x_{n}\right)_{n} \in l_{p}$, não é $\omega$-seqüencialmente contínuo em 0 .

b) $\operatorname{Dados} N \in \mathbb{N} e\left(\lambda_{k}\right)_{k} \in l_{\infty} \backslash c_{00} \operatorname{com} \lambda_{k} \nrightarrow-1$, seja $P: c_{0} \longrightarrow l_{\infty}$ dado por $P(x)=\sum_{k=1}^{\infty}\left(1+\lambda_{k}\right) x_{k}^{N} e_{k}$ para cada $x=\left(x_{k}\right)_{k} \in c_{0}$, temos que $P$ não é $\omega$-seqüencialmente contínuo em 0.

De fato: Para a base canônica $\left(e_{n}\right)_{n}$, temos que $e_{n} \stackrel{\omega}{\longrightarrow} 0$ em $c_{0}$ e $l_{p}$. No caso (a), $P\left(e_{n}\right)=e_{n}$ para cada $n \in \mathbb{N}$. E no caso (b) $P\left(e_{n}\right)=\left(1+\lambda_{n}\right) e_{n}$ para cada $n \in \mathbb{N}$. 
Veremos a seguir, proposição 3.1.2, que os polinômios do exemplo 3.1.1 não são fracamente seqüencialmente contínuo em nenhum ponto do espaço em questão.

Dados $E, F$ espaços de Banach, $N \in \mathbb{N}$ e $P: E \longrightarrow F$ um polinômio $N$-homogêneo contínuo, estudaremos o seguinte conjunto:

$$
C(P)=\{x \in E: P \text { é } \omega-\text { seqüencialmente contínuo em } x\} .
$$

Na proposição abaixo vamos apresentar algumas propriedades básicas do conjunto $C(P)$. Tais resultados são análogos aos apresentados por Aron e Dimant em [5], proposição 1 , para o caso $F=\mathbb{K}$.

Primeiramente vamos definir os seguintes polinômios que utilizaremos a seguir:

Dado $P \in \mathcal{P}\left({ }^{N} E ; F\right)$, para cada $j \in\{0, \ldots, N\}$ definamos $\Phi_{j}: E \longrightarrow \mathcal{P}\left({ }^{N-j} E ; F\right)$ da seguinte maneira: Para cada $x \in E, \Phi_{j}(x): E \longrightarrow F, \Phi_{j}(x)(y)=A\left(x^{j}, y^{N-j}\right)$ para todo $y \in E$, onde $A$ é a aplicação $N$-linear simétrica associada ao polinômio $P$.

Proposição 3.1.2. Seja $P \in \mathcal{P}\left({ }^{N} E ; F\right)$. As seguintes afirmações valem:

1. $C(P)$ é um subconjunto fechado de $E$.

2. Se $x \in C(P)$ então $\lambda x \in C(P)$ para todo $\lambda \in \mathbb{K}$. Em particular, se $C(P) \neq \emptyset$ então $0 \in C(P)$.

3. $C(P)=\cap_{j=0}^{N-1}\left\{x \in E: \Phi_{j}(x) \in \mathcal{P}_{w s c(0)}\left({ }^{N-j} E ; F\right)\right\}$.

Demonstração: A demonstração é análoga ao caso $F=\mathbb{K}$.

Observação 3.1.3. Dado $N \in \mathbb{N}, N \geq 2$, seja $P \in \mathcal{P}\left({ }^{N} E ; F\right)$ tal que o conjunto $\left\{x \in E: \Phi_{1}(x) \in \mathcal{P}_{w s c(0)}\left({ }^{N-1} E ; F\right)\right\} \neq \emptyset$. Então $\left\{x \in E: \Phi_{1}(x) \in \mathcal{P}_{w s c(0)}\left({ }^{N-1} E ; F\right)\right\}$ é um subespaço, uma vez que $\left\{x \in E: \Phi_{1}(x) \in \mathcal{P}_{w s c(0)}\left({ }^{N-1} E ; F\right)\right\}=\Phi_{1}^{-1}\left(\mathcal{P}_{w s c(0)}\left({ }^{N-1} E ; F\right)\right)$. Isto é, imagem inversa do subespaço $\mathcal{P}_{\text {wsc }(0)}\left({ }^{N-1} E ; F\right)$ pela aplicação linear $\Phi_{1}$.

No próximo exemplo vamos aplicar a proposição 3.1.2. 
Exemplos 3.1.4. Sejam $p, q \in] 1, \infty\left[\right.$ e suponhamos $q u e ~ q<p$. Tomemos $N=\left\lfloor\frac{p}{q}\right\rfloor:=o$ menor inteiro maior ou igual a $\frac{p}{q}$. Temos:

1. Para cada $1 \leq j \leq(N-1)$ e cada $P \in \mathcal{P}\left({ }^{j} l_{p} ; l_{q}\right)$ tem-se que $C(P)=l_{p}$.

2. Para cada $P \in \mathcal{P}\left({ }^{N} l_{p} ; l_{q}\right)$ ou $C(P)=\emptyset$ ou $C(P)=l_{p}$.

3. Para cada $P \in \mathcal{P}\left({ }^{N+1} l_{p} ; l_{q}\right)$ ou $C(P)=\emptyset$ ou $C(P)$ é um subespaço.

De fato: Alencar e Floret em [2] demonstram que dado $j \in \mathbb{N}$, vale $\mathcal{P}\left({ }^{j} l_{p} ; l_{q}\right)=\mathcal{P}_{w s c}\left({ }^{j} l_{p} ; l_{q}\right)$ se e somente se $j q<p$ (teorema 4.2 de [2]). Aqui, para cada $1 \leq j \leq(N-1)$ temos que $p>j q$ pois $p>(N-1) q$ e então, $\mathcal{P}\left({ }^{j} l_{p} ; l_{q}\right)=\mathcal{P}_{w s c}\left({ }^{j} l_{p} ; l_{q}\right)$ $\forall 1 \leq j \leq(N-1)$. Agora, pela proposição 3.1.2 seguem (2) e (3).

É bem conhecido que se o espaço $E$ tem a propriedade de Dunford-Pettis e o espaço $F$ é tal que cada $T \in \mathcal{L}(E ; F)$ é fracamente compacta, vale a igualdade $\mathcal{P}\left({ }^{N} E ; F\right)=\mathcal{P}_{w s c}\left({ }^{N} E ; F\right) \forall N \in \mathbb{N}$ (ver por exemplo [25] teorema 3.45).

A próxima proposição nos auxiliará a exibir mais alguns exemplos de espaços de Banach onde os polinômios são fracamente seqüencialmente contínuos. Na demonstração usaremos o seguinte fato:

Dado $N \in \mathbb{N}$, existem um único espaço de Banach, que é denotado por $\underset{N, s, \pi}{\otimes} E$, e um único $i_{N} \in \mathcal{P}\left({ }^{N} E ; \underset{N, s, \pi}{\otimes} E\right), i_{N}(x)=x \otimes . N \otimes x$, tal que para qualquer espaço $F$ e qualquer $P \in \mathcal{P}\left({ }^{N} E ; F\right)$ existe uma única $i_{N}^{*}(P) \in \mathcal{L}\left(\bigotimes_{N, s, \pi} E ; F\right)$ tal que o seguinte diagrama $\begin{array}{clll}E & \stackrel{P}{\longrightarrow} & F \\ i_{N} \searrow & \underset{\bigotimes_{N, s, \pi} E}{\longrightarrow} & \nearrow i_{N}^{*}(P) \text { comuta, ou seja, } i_{N}^{*}(P) \circ i_{N}(x)=P(x) .\end{array}$

Proposição 3.1.5. Sejam E, F espaços de Banach e $N \in \mathbb{N}$. Se $\mathcal{P}\left({ }^{N} E ; F\right)=\mathcal{P}_{\text {wsc }}\left({ }^{N} E ; F\right)$ então $\mathcal{P}\left({ }^{N} E\right)=\mathcal{P}_{\text {wsc }}\left({ }^{N} E\right)$. Reciprocamente, se $F$ é de Schur, a igualdade $\mathcal{P}\left({ }^{N} E\right)=\mathcal{P}_{\text {wsc }}\left({ }^{N} E\right)$ implica que $\mathcal{P}\left({ }^{N} E ; F\right)=\mathcal{P}_{w s c}\left({ }^{N} E ; F\right)$. 
Demonstração: Suponhamos que $\mathcal{P}\left({ }^{N} E ; F\right)=\mathcal{P}_{\text {wsc }}\left({ }^{N} E ; F\right)$. Dado $P \in \mathcal{P}\left({ }^{N} E\right)$, sejam $x \in E$ e $\left(x_{n}\right)_{n}$ uma seqüência em $E$ tal que $x_{n} \stackrel{\omega}{\longrightarrow} x$. Tomemos $b \in F$ com $\|b\|=$ 1 e definamos $Q: E \longrightarrow F$ por $Q(y)=(P \otimes b)(y):=P(y) \cdot b$ para cada $y \in E$. Temos que $Q \in \mathcal{P}\left({ }^{N} E ; F\right)$. Por hipótese, $Q\left(x_{n}\right) \stackrel{n \rightarrow \infty}{\longrightarrow} Q(x)$, ou seja, $P\left(x_{n}\right) \stackrel{n \rightarrow \infty}{\longrightarrow} P(x)$. Logo, $\mathcal{P}\left({ }^{N} E\right)=\mathcal{P}_{w s c}\left({ }^{N} E\right)$.

Agora suponhamos que $F$ é de Schur e $\mathcal{P}\left({ }^{N} E\right)=\mathcal{P}_{\text {wsc }}\left({ }^{N} E\right)$. Dado $P \in \mathcal{P}\left({ }^{N} E ; F\right)$, tomemos $x \in E$ e $\left(x_{n}\right)_{n}$ uma seqüência em $E$ tal que $x_{n} \stackrel{\omega}{\longrightarrow} x$. Por hipótese, $Q\left(x_{n}\right) \stackrel{n \rightarrow \infty}{\longrightarrow} Q(x)$ para cada $Q \in \mathcal{P}\left({ }^{N} E\right)$. Como $\left(\hat{\otimes}_{N, s, \pi} E\right)^{\prime} \cong \mathcal{P}\left({ }^{N} E\right)$, segue que $x_{n} \otimes \stackrel{(N)}{\cdots} \otimes x_{n} \stackrel{\omega}{\longrightarrow} x \otimes \stackrel{(N)}{\cdots} \otimes x \mathrm{em}$ $\hat{\bigotimes}_{N, s, \pi} E$. Sendo $i_{N}^{*}(P)$ linear e contínua, temos que $i_{N}^{*}(P)\left(x_{n} \otimes(N) \otimes x_{n}\right) \stackrel{\omega}{\longrightarrow} i_{N}^{*}(P)(x \otimes(N) \otimes x)$ em $F$, ou seja, $P\left(x_{n}\right) \stackrel{\omega}{\longrightarrow} P(x)$ em $F$. Como, por hipótese, $F$ é de Schur, temos que $P\left(x_{n}\right) \stackrel{n \rightarrow \infty}{\longrightarrow} P(x)$. E assim, $\mathcal{P}\left({ }^{N} E ; F\right)=\mathcal{P}_{w s c}\left({ }^{N} E ; F\right)$.

Observação 3.1.6. Sejam E, F espaços de Banach tais que $E$ tem a propriedade de Dunford-Pettis e F é de Schur. Então $\mathcal{P}\left({ }^{N} E ; F\right)=\mathcal{P}_{\text {wsc }}\left({ }^{N} E ; F\right)$ cada $N \in \mathbb{N}$.

Observamos que a condição de o espaço $F$ ser de Schur é necessária, pois pela proposição 3.1.2, para o polinômio $P: c_{0} \longrightarrow l_{\infty}$ dado por $P(x)=\sum_{k=1}^{\infty} x_{k}^{N} e_{k}$ para cada $x=\left(x_{k}\right)_{k} \in c_{0}$ temos que $C(P)=\emptyset$.

Corolário 3.1.7. Sejam E, F espaços de Banach tais que F é de Schur e $\mathcal{P}\left({ }^{j} E\right)=\mathcal{P}_{\text {wsc }}\left({ }^{j} E\right)$ para cada $j \in\{1, \ldots, N-1\}$ e $\mathcal{P}\left({ }^{N} E\right) \neq \mathcal{P}_{\text {wsc }}\left({ }^{N} E\right)$. Então:

1. Para cada $r<N$ e cada $P \in \mathcal{P}\left({ }^{r} E ; F\right)$ temos que $C(P)=E$.

2. Para cada $P \in \mathcal{P}\left({ }^{N} E ; F\right)$ temos que $C(P)=\emptyset$ ou $C(P)=E$.

3. Para cada $P \in \mathcal{P}\left({ }^{N+1} E ; F\right)$ temos que $C(P)=\emptyset$ ou $C(P)$ é um subespaço de $E$.

Demonstração: Segue das proposições 3.1 .5 e 3.1.2, de modo análogo ao caso $F=\mathbb{K}$.

Exemplo 3.1.8. Dado $p \in] 1, \infty[$ tomemos $N=\lfloor p\rfloor:=$ o menor inteiro maior ou igual a p. Para cada $r \leq N-1$, pelo corolário 3.1.7(1) temos que $\mathcal{P}\left({ }^{r} l_{p} ; l_{1}\right)=\mathcal{P}_{\text {wsc }}\left({ }^{r} l_{p} ; l_{1}\right)$. Em 
particular, $\mathcal{P}\left({ }^{r} l_{q} ; l_{1}\right)=\mathcal{P}_{\text {wsc }}\left({ }^{r} l_{q} ; l_{1}\right)$ para todo $q \geq p$ e cada $r \leq N-1$. Também, pelo item (2) do corolário 3.1.7 temos que $\mathcal{P}_{w s c}\left({ }^{N} l_{p} ; l_{1}\right)=\mathcal{P}_{w s c(0)}\left({ }^{N} l_{p} ; l_{1}\right)$.

Observamos também que, com argumentação análoga à proposição 4 de [5] temos a seguinte:

Proposição 3.1.9. Sejam E, F espaços de Banach.

i) Dado $N \in \mathbb{N}$, seja $P \in \mathcal{P}\left({ }^{N} E ; F\right)$ tal que $C(P)$ contém $N$ hiperplanos, então $C(P)=E$. Além disso, se $\mathcal{L}(E ; F)=\mathcal{L}_{w s c}(E ; F)$ e $C(P)$ contém $N-1$ hiperplanos, então $C(P)=E$

ii) Dado $s \in \mathbb{N}$, suponhamos que $\mathcal{P}\left({ }^{r} E ; F\right)=\mathcal{P}_{\text {wsc }(0)}\left({ }^{r} E ; F\right) \forall r \leq s$. Para $N \in \mathbb{N}$ com $N \geq s+1$ seja $P \in \mathcal{P}\left({ }^{N} E ; F\right)$ tal que $C(P)$ contém $(N-s)$ hiperplanos. Então $C(P)=E$.

Observação 3.1.10. Na proposição 3.1.9, a hipótese de $\mathcal{L}(E ; F)=\mathcal{L}_{\text {wsc }}(E ; F)$ é condição necessária. Para $N \geq 2$ e $P: c_{0} \longrightarrow l_{\infty}$ dado por $P(x)=x_{1} \cdot \ldots \cdot x_{N-1} \cdot \sum_{i=1}^{\infty} x_{i} e_{i}$ para cada $x=\sum_{i=1}^{\infty} x_{i} e_{i} \in E$, temos que $C(P)=\left\{x=\left(x_{i}\right)_{i} \in c_{0}: x_{1}=0\right.$ ou $\ldots$ ou $\left.x_{N-1}=0\right\}$.

De fato: $\operatorname{Sejam} x=\left(x_{i}\right)_{i} \in c_{0} \operatorname{com} x_{i}=0$ para algum $i \in\{1, \ldots, N-1\}$ e $\left(x_{n}\right)_{n} \subset c_{0}$, $x_{n}=\left(x_{n i}\right)_{i}$, tal que $x_{n} \stackrel{\omega}{\longrightarrow} x$. Então $\left(x_{n}\right)_{n}$ é limitada e para cada $i \in \mathbb{N}$ temos que $x_{n i} \stackrel{n \rightarrow \infty}{\longrightarrow} x_{i}$. Para cada $n \in \mathbb{N}$ temos que

$$
\left\|P\left(x_{n}\right)-P(x)\right\|_{\infty}=\sup _{i}\left|x_{n 1} \cdot \ldots \cdot x_{n(N-1)} \cdot x_{n i}\right| \leq\left|x_{n 1}\right| \cdot \ldots \cdot\left|x_{n(N-1)}\right| \cdot\left\|x_{n}\right\|_{\infty} .
$$

E desta maneira, $\left\|P\left(x_{n}\right)-P(x)\right\|_{\infty} \stackrel{n \rightarrow \infty}{\longrightarrow} 0$.

Seja $x \in c_{0}, x=\left(x_{i}\right)_{i}$, suponhamos que $x_{1}, \ldots, x_{N-1}$ são todos não nulos e tomemos a seqüência $\left(e_{n+N-1}+x\right)_{n}$. Temos que $e_{n+N-1}+x \stackrel{\omega}{\longrightarrow} x$ e $\left\|P\left(e_{n+N-1}+x\right)-P(x)\right\|_{\infty}=\left|x_{1} \cdot \ldots \cdot x_{N-1}\right|>0$.

Dados $P \in \mathcal{P}\left({ }^{N} E\right)$ e $Q \in \mathcal{P}\left({ }^{N} E\right)$, Aron e Dimant em [5] demonstram que

$$
C(P \cdot Q)=[C(P) \cap C(Q)] \cup\left[C(P) \cap P^{-1}(0)\right] \cup\left[C(Q) \cap Q^{-1}(0)\right] .
$$


Para polinômios com valores vetoriais podemos efetuar o produto de dois polinômios se o espaço $F$ for uma álgebra de Banach, ou seja, $F$ é uma álgebra tal que a norma satisfaz $\|x \cdot y\| \leq\|x\| \cdot\|y\|$.

O espaço de Banach $C(K)$ com $K$ compacto e Hausdorff é uma álgebra de Banach com identidade munido das operações usuais.

O espaço $\left(l_{\infty}, \cdot\right)$ com $\cdot$ sendo o produto pontual é uma álgebra de Banach com identidade. Também, o espaço $c_{0}$ é uma subálgebra fechada de $l_{\infty}$, e portanto é uma álgebra de Banach, mas não admite identidade.

Agora vamos considerar os seguintes espaços:

$$
\mathcal{P}_{f}\left({ }^{N} E ; F\right)=\left\{P \in \mathcal{P}\left({ }^{N} E ; F\right): P=\sum_{i=1}^{k} \phi_{i}^{N} \cdot b_{i}, \phi_{i} \in E^{\prime}, b_{i} \in F\right\}
$$

onde para cada $\phi \in E^{\prime}$ e cada $b \in F,\left(\phi^{N} \cdot b\right)(x)=(\phi(x))^{N} \cdot b \forall x \in E$. E se o espaço $F$ é uma álgebra de Banach,

$$
\mathbb{P}_{f}\left({ }^{N} E ; F\right)=\left\{P \in \mathcal{P}\left({ }^{N} E ; F\right): P=\sum_{i=1}^{k} T_{i}^{N}, T_{i} \in \mathcal{L}(E ; F)\right\}
$$

onde para cada $T \in \mathcal{L}(E ; F), T^{N}(x)=T(x) \cdot \stackrel{(N)}{*} \cdot T(x)$ para cada $x \in E$.

De imediato, temos que $C(P)=E$ para cada $P \in{\overline{\mathcal{P}_{f}\left({ }^{N} E ; F\right)}}^{\|\cdot\|}$, pois $\mathcal{P}_{f}\left({ }^{N} E ; F\right) \subset$ $\mathcal{P}_{w s c}\left({ }^{N} E ; F\right)$. Agora suponhamos que $F$ é uma álgebra de Banach. Seja $P \in \overline{\mathbb{P}_{f}\left({ }^{N} E ; F\right)}{ }^{\|\cdot\|}$, se $\left(P_{m}\right)_{m} \subset \mathbb{P}_{f}\left({ }^{N} E ; F\right)$ é tal que $P_{m} \stackrel{m \rightarrow \infty}{\longrightarrow} P$ temos que $\cap_{m=1}^{\infty} C\left(P_{m}\right) \subset C(P)$. Além disso, se $\mathcal{L}(E ; F)=\mathcal{L}_{\text {wsc }}(E ; F)$, tem-se $C(P)=E$.

Dados $E, F$ espaços de Banach, e suponhamos que $F$ é uma álgebra de Banach. Então, dados polinômios $P \in \mathcal{P}\left({ }^{N} E ; F\right)$ e $Q \in \mathcal{P}\left({ }^{M} E ; F\right)$ denotaremos o produto dos polinômios $P$ e $Q, P \cdot Q \in \mathcal{P}\left({ }^{N+M} E ; F\right)$ por $(P \cdot Q)(x):=P(x) \cdot Q(x)$ para cada $x \in E$. Com uma argumentação bem simples temos que:

$$
[C(P) \cap C(Q)] \cup\left[C(P) \cap P^{-1}(0)\right] \cup\left[C(Q) \cap Q^{-1}(0)\right] \subset C(P \cdot Q) .
$$

Em geral, para o caso vetorial, não temos a igualdade em 3.2 como podemos ver no seguinte exemplo: 
Exemplo 3.1.11. Dado $q \in] 1, \infty\left[\right.$, em $l_{q}$ podemos definir $x \cdot y=\sum_{n=1}^{\infty} \alpha_{n} \beta_{n} e_{n}$ para cada $x=\sum_{n=1}^{\infty} \alpha_{n} e_{n} \in l_{q}$ e $y=\sum_{n=1}^{\infty} \beta_{n} e_{n} \in l_{q}$. Temos então, que $\left(l_{q}, \cdot,\|\cdot\|\right)$ é uma álgebra de Banach. Dado $p \in] q, \infty\left[\right.$, tomemos $N=\left\lfloor\frac{p}{q}\right\rfloor$. Dados $\left(\lambda_{i}\right)_{i} \in l_{\infty} \backslash c_{0} e\left(\xi_{i}\right)_{i} \in l_{1}$ sejam $P, Q: l_{p} \longrightarrow l_{q}$ os polinômios $N$-homogêneos definidos por $P(x)=\sum_{i=1}^{\infty} \xi_{i} x_{i}^{N} e_{i}$ e $Q(x)=\sum_{i=1}^{\infty} \lambda_{i} x_{i}^{N} e_{i}$ para cada $x=\sum_{i=1}^{\infty} x_{i} e_{i} \in l_{p}$. Temos que $C(P)=l_{p}, C(Q)=\emptyset e$ $C(P \cdot Q)=l_{p}$.

De fato: Como $e_{n} \stackrel{\omega}{\longrightarrow} 0$ e $Q\left(e_{n}\right)=\lambda_{n} e_{n}$ para cada $n \in \mathbb{N}$, temos que $C(Q)=\emptyset$. Para cada $m \in \mathbb{N}$, definamos os polinômios $P_{m}, R_{m}: l_{p} \longrightarrow l_{q}$ por $P_{m}(x)=\sum_{i=1}^{m} \xi_{i} x_{i}^{N} e_{i}$ e $R_{m}(x)=\sum_{i=1}^{m} \xi_{i} \lambda_{i} x_{i}^{2 N} e_{i}$ para cada $x=\sum_{i=1}^{\infty} x_{i} e_{i} \in l_{p}$. Temos que, $P_{m} \in \mathcal{P}_{f}\left({ }^{N} l_{p} ; l_{q}\right)$, $R_{m} \in \mathcal{P}_{f}\left({ }^{2 N} l_{p} ; l_{q}\right), P_{m} \stackrel{m \rightarrow \infty}{\longrightarrow} P$ e $R_{m} \stackrel{m \rightarrow \infty}{\longrightarrow} P \cdot Q$, e assim, $C(P)=l_{p}$ e $C(P \cdot Q)=l_{p}$.

No que segue, vamos utilizar a fórmula 3.2 para fazer afirmações para alguns tipos de polinômios.

Proposição 3.1.12. Sejam E, $F$ espaços de Banach. Suponhamos que existe $N \in \mathbb{N}$ tal que $\mathcal{P}\left({ }^{r} E ; F\right)=\mathcal{P}_{w s c}\left({ }^{r} E ; F\right) \forall r<N$ e $\mathcal{P}\left({ }^{N} E ; F\right) \neq \mathcal{P}_{w s c}\left({ }^{N} E ; F\right)$. Então, para cada $M \in \mathbb{N}$ e cada $Q \in \mathcal{P}_{\text {wsc }}\left({ }^{M} E\right)$ existe $R \in \mathcal{P}\left({ }^{M+N} E ; F\right)$ tal que $C(R)=Q^{-1}(0)$.

Demonstração: $\quad$ Sejam $M \in \mathbb{N}, Q \in \mathcal{P}_{\text {wsc }}\left({ }^{M} E\right)$ e $P \in \mathcal{P}\left({ }^{N} E ; F\right) \backslash \mathcal{P}_{w s c}\left({ }^{N} E ; F\right)$. Tomemos o polinômio $R \in \mathcal{P}\left({ }^{M+N} E ; F\right)$ definido por $R(x):=Q(x) \cdot P(x)$ para cada $x \in E$. Pela fórmula 3.2 temos que

$$
[C(P) \cap C(Q)] \cup\left[C(P) \cap P^{-1}(0)\right] \cup\left[C(Q) \cap Q^{-1}(0)\right] \subset C(P \cdot Q) .
$$

Pela proposição 3.1.2, itens 2 e 3 , segue que $C(P)=\emptyset$ e como $C(Q)=E$ temos que $Q^{-1}(0) \subset C(R)$.

Seja $x \in E$ tal que $|Q(x)|>0$. Como $C(P)=\emptyset$, existe uma seqüência $\left(x_{n}\right)_{n}$ tal que

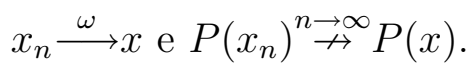

Sejam $A_{1} \in \mathcal{L}_{s}\left({ }^{M} E\right), A_{2} \in \mathcal{L}_{s}\left({ }^{N} E ; F\right)$ as aplicações associadas aos polinômios $Q$ e $P$ 
respectivamente.

Para cada escolha $\left\{i_{1}, \ldots, i_{M}\right\}$ com $1 \leq i_{1}<i_{2}<\ldots<i_{M} \leq M+N$, vamos denotar por $\left\{a_{1}, \ldots, a_{N}\right\}:=\{1, \ldots, M+N\} \backslash\left\{i_{1}, \ldots, i_{M}\right\}$. A aplicação $(M+N)$-linear simétrica associada ao polinômio $R$ é:

$$
\begin{aligned}
& A\left(x_{1}, \ldots, x_{M}, x_{M+1}, \ldots, x_{M+N}\right)= \\
& =\frac{M ! N !}{(M+N) !} \sum_{1 \leq i_{1}<i_{2}<\ldots<i_{M} \leq M+N} A_{1}\left(x_{i_{1}}, \ldots, x_{i_{M}}\right) \cdot A_{2}\left(x_{a_{1}}, \ldots, x_{a_{N}}\right) .
\end{aligned}
$$

Pela proposição 3.1 .2 e por hipótese temos que

$$
C(R)=\cap_{j=0}^{M}\left\{y \in E: \Phi_{j}(y) \in \mathcal{P}_{w s c(0)}\left({ }^{M+N-j} E ; F\right)\right\}
$$

Suponhamos que $x \in C(R)$. Como $x_{n}-x \stackrel{\omega}{\longrightarrow} 0$, temos que

$\Phi_{M}(x)\left(x_{n}-x\right) \stackrel{n \rightarrow \infty}{\longrightarrow} \Phi_{M}(x)(0)=A\left(x^{M}, 0^{N}\right)=0$. Para cada $y \in E$ temos:

Se $M \leq N, \Phi_{M}(x)(y)=A\left(x^{M}, y^{N}\right)=$

$$
\frac{M ! N !}{(M+N) !} \sum_{i=0}^{M} \frac{M ! N !}{(N-M+i) ![(M-i) !]^{2} i !} A_{1}\left(x^{i}, y^{M-i}\right) \cdot A_{2}\left(x^{N-i}, y^{i}\right) .
$$

Se $M>N, \Phi_{M}(x)(y)=A\left(x^{M}, y^{N}\right)=$

$$
\frac{M ! N !}{(M+N) !} \sum_{i=M-N}^{M} \frac{M ! N !}{(N-M+i) ![(M-i) !]^{2} i !} A_{1}\left(x^{i}, y^{M-i}\right) \cdot A_{2}\left(x^{M-i}, y^{N-M+i}\right) .
$$

E assim, para $M \leq N, \Phi_{M}(x)\left(x_{n}-x\right)=\frac{M ! N !}{(M+N) !} Q(x) \cdot P\left(x_{n}-x\right)+$

$$
\frac{M ! N !}{(M+N) !} \sum_{i=0}^{M-1} \frac{M ! N !}{(N-M+i) ![(M-i) !]^{2} i !} A_{1}\left(x^{i},\left(x_{n}-x\right)^{M-i}\right) \cdot A_{2}\left(x^{N-i},\left(x_{n}-x\right)^{i}\right),
$$

e para $M>N, \Phi_{M}(x)\left(x_{n}-x\right)=\frac{M ! N !}{(M+N) !} Q(x) \cdot P\left(x_{n}-x\right)+$

$$
\frac{M ! N !}{(M+N) !} \sum_{i=M-N}^{M-1} \frac{M ! N !}{(N-M+i) ![(M-i) !]^{2} i !} A_{1}\left(x^{i},\left(x_{n}-x\right)^{M-i}\right) \cdot A_{2}\left(x^{M-i},\left(x_{n}-x\right)^{N-M+i}\right) .
$$

No caso $M \leq N$, para cada $i=0, \ldots, M-1$, temos que: $A_{2}\left(x^{N-i},\left(x_{n}-x\right)^{i}\right) \stackrel{n \rightarrow \infty}{\longrightarrow} 0$ (por hipótese) e $A_{1}\left(x^{i},\left(x_{n}-x\right)^{M-i}\right) \stackrel{n \rightarrow \infty}{\longrightarrow} 0$ (por hipótese e aplicando a proposição 3.1.5). E assim, segue que $A_{1}\left(x^{i},\left(x_{n}-x\right)^{M-i}\right) \cdot A_{2}\left(x^{N-i},\left(x_{n}-x\right)^{i}\right) \stackrel{n \rightarrow \infty}{\longrightarrow} 0$. Desta maneira, temos que 
$P\left(x_{n}-x\right) \stackrel{n \rightarrow \infty}{\longrightarrow} 0$, pois $|Q(x)|>0$.

E quando $M>N$, temos: Para cada $i=M-N, \ldots, M-1, A_{1}\left(x^{i},\left(x_{n}-x\right)^{M-i}\right) \stackrel{n \rightarrow \infty}{\longrightarrow} 0$ pois $x \in C(Q)$ e para $i=M-N+1, \ldots, M-1, A_{2}\left(x^{M-i},\left(x_{n}-x\right)^{N-M+i}\right) \stackrel{n \rightarrow \infty}{\longrightarrow} 0$ (por hipótese). E neste caso, também segue que $P\left(x_{n}-x\right) \stackrel{n \rightarrow \infty}{\longrightarrow} 0$.

Para cada $n \in \mathbb{N}, P\left(x_{n}\right)-P(x)=P\left(x_{n}-x\right)+\sum_{j=1}^{N-1} A_{2}\left(x^{j},\left(x_{n}-x\right)^{N-j}\right)$ e assim, $P\left(x_{n}\right)-P(x) \stackrel{n \rightarrow \infty}{\longrightarrow} 0$, uma contradição. Portanto $C(R)=Q^{-1}(0)$.

Proposição 3.1.13. Sejam E, F espaços de Banach.

1. Suponhamos que existe $N \in \mathbb{N}$ tal que $\mathcal{P}\left({ }^{r} E ; F\right)=\mathcal{P}_{\text {wsc }}\left({ }^{r} E ; F\right) \forall r<N$ e $\mathcal{P}\left({ }^{N} E ; F\right) \neq \mathcal{P}_{w s c}\left({ }^{N} E ; F\right)$. Então:

i) Dado um hiperplano $H$, para cada $M \in \mathbb{N}$ existe $Q \in \mathcal{P}\left({ }^{N+M} E ; F\right)$ tal que $C(Q)=H$.

ii) Dados $H_{1}, \ldots, H_{M}$ hiperplanos de $E$ existe $Q \in \mathcal{P}\left({ }^{N+M} E ; F\right)$ tal que $C(Q)=H_{1} \cup \ldots \cup H_{M}$.

2. Se $\mathcal{L}(E ; F) \neq \mathcal{L}_{w s c}(E ; F)$, então dados $H_{1}, \ldots, H_{N}$ hiperplanos de $E$ existe $P \in \mathcal{P}\left({ }^{N+1} E ; F\right)$ tal que $C(P)=H_{1} \cup \ldots \cup H_{N}$.

Demonstração: (1)(i) Sejam $\phi \in E^{\prime}$ tal que $\phi^{-1}(0)=H, M \in \mathbb{N}$ e $P \in \mathcal{P}\left({ }^{N} E ; F\right) \backslash$ $\mathcal{P}_{\text {wsc }}\left({ }^{N} E ; F\right)$. Tomemos $Q \in \mathcal{P}\left({ }^{M+N} E ; F\right)$ definido por $Q(x):=\phi^{M}(x) \cdot P(x)$. Usando a mesma técnica de demonstração da proposição 3.1 .12 segue que $C(Q)=\phi^{-1}(0)$. Para (ii), sejam $\phi_{1}, \ldots, \phi_{M} \in E^{\prime}$ tais que $\phi^{-1}(0)=H_{1}, \ldots, \phi_{M}^{-1}(0)=H_{M}$. Tomemos $Q \in$ $\mathcal{P}\left({ }^{M+N} E ; F\right)$ definido por $Q(x):=\phi_{1}(x) . \ldots \cdot \phi_{M}(x) \cdot P(x) \operatorname{com} P \in \mathcal{P}\left({ }^{N} E ; F\right) \backslash \mathcal{P}_{w s c}\left({ }^{N} E ; F\right)$. Agora usando a mesma técnica de demonstração da proposição 3.1.12 temos que $C(Q)=$ $H_{1} \cup \ldots \cup H_{M}$.

De maneira análoga ao item (ii) segue (2).

Exemplo 3.1.14. 1. Sejam $p \in] 1, \infty\left[, M \in \mathbb{N}\right.$ e $P \in \mathcal{P}_{w s c}\left({ }^{M} l_{p}\right)$. Dado $Q_{1} \in \mathcal{P}\left(\lfloor p\rfloor l_{p} ; l_{1}\right)$, para o polinômio $R_{1}=P \cdot Q_{1}$ temos que ou $C\left(R_{1}\right)=l_{p}$ ou $C\left(R_{1}\right)=P^{-1}(0)$. Se $p>q$, 
dado $Q_{2} \in \mathcal{P}\left({ }^{\left\lfloor\frac{p}{q}\right\rfloor} l_{p} ; l_{q}\right)$, para o polinômio $R_{2}=P \cdot Q_{2}$ temos que ou $C\left(R_{2}\right)=l_{p}$ ou $C\left(R_{2}\right)=P^{-1}(0)$.

2. Dado um subespaço fechado $\left.S \subset l_{p}, p \in\right] q, \infty[$, para cada $M \in \mathbb{N}$ existe uma

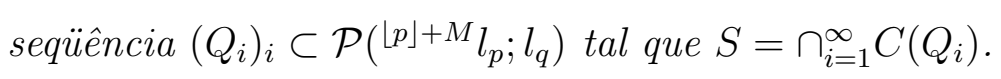

De fato: Para o item (1) usamos os exemplos 3.1.4 e 3.1.8 e técnica de demonstração da proposição 3.1.12. E para o item (2), usamos o exemplo 3.1.4, proposição 12(a) de [5] e proposição 3.1 .13 (item 1i).

No que segue, temos por objetivo apresentar alguns exemplos de polinômios $P \in{\overline{\mathbb{P}_{f}\left({ }^{N} E ; F\right)}}^{\|\cdot\|}$ tais que $C(P)=E$ onde a álgebra de Banach $F$ não é Schur.

Exemplos 3.1.15. Dado $p \in] 1, \infty\left[\right.$, consideremos $l_{p}$ munido com a operação produto e a função norma definidas a seguir: Para cada $x=\sum_{n=1}^{\infty} \alpha_{n} e_{n}, y=\sum_{n=1}^{\infty} \beta_{n} e_{n} \in l_{q}$, definamos $x \odot y:=\beta_{1} \sum_{n=1}^{\infty} \alpha_{n} e_{n}+\alpha_{1} \sum_{n=2}^{\infty} \beta_{n} e_{n}$ e $\||x|\|:=\left|\alpha_{1}\right|+\left\|\sum_{n=2}^{\infty} \alpha_{n} e_{n}\right\|_{q}$. Temos que $\left(l_{q}, \odot,\||\cdot|\| \mid\right)$ é uma álgebra de Banach com identidade $e_{1}$ e a norma ||$|\cdot|||$ é equivalente à norma original (ver [28], pg 523). Sejam $\left(\lambda_{k}\right)_{k} \in l_{\infty} e N \in \mathbb{N}$.

1. Para o polinômio $P: l_{p} \longrightarrow l_{p}$ definido por $P(x)=\sum_{i=1}^{\infty} \lambda_{i} x_{i}^{N} e_{i}$ para cada $x=\sum_{i=1}^{\infty} x_{i} e_{i} \in l_{p}$, temos: $S e\left(\lambda_{k}\right)_{k} \in l_{\infty} \backslash c_{0}, P \notin \overline{\mathcal{P}_{f}\left({ }^{N} l_{p} ; l_{p}\right)}\|\cdot\|$. Ese $\left(\lambda_{k}\right)_{k} \in l_{p}$, $P \in \overline{\mathbb{P}_{f}\left({ }^{N} l_{p} ; l_{p}\right)}\|\cdot\|$ e $C(P)=l_{p}$.

2. Em $l_{1}$ consideremos o produto definido como acima. Se $N \geq$ p, para o polinômio $P: l_{p} \longrightarrow l_{1}$ definido por $P(x)=\sum_{i=1}^{\infty} \lambda_{i} x_{i}^{N} e_{i}$ para cada $x=\sum_{i=1}^{\infty} x_{i} e_{i} \in l_{p}$, temos que $P \notin \overline{\mathcal{P}_{f}\left({ }^{N} l_{p} ; l_{1}\right)}{ }^{\|\cdot\|}$ se $\left(\lambda_{k}\right)_{k} \in l_{\infty} \backslash c_{0}$ e se $\left(\lambda_{k}\right)_{k} \in l_{1} P \in \overline{\mathbb{P}_{f}\left({ }^{N} l_{p} ; l_{1}\right)}\|\cdot\|$ conseqüentemente, $C(P)=l_{p}$.

De fato: (1) Para a base canônica, $e_{n} \stackrel{\omega}{\longrightarrow} 0$ e para cada $n \in \mathbb{N}, P\left(e_{n}\right)=\lambda_{n} e_{n}$. Se $\left(\lambda_{k}\right)_{k} \notin c_{0}$ temos que $P\left(e_{n}\right) \nrightarrow 0$ e assim, pela proposição 3.1.2 temos que $C(P)=\emptyset$. Desta maneira, segue que $P \notin \overline{\mathcal{P}_{f}\left({ }^{N} l_{p} ; l_{p}\right)}\|\cdot\|$. Para o caso $\left(\lambda_{k}\right)_{k} \in l_{p}$, para cada $m \in \mathbb{N}$, 
definamos $P_{m}: l_{p} \longrightarrow l_{p}$ por $P_{m}(x)=\sum_{i=1}^{m} \lambda_{i} x_{i}^{N} e_{i}$ para cada $x=\left(x_{i}\right)_{i} \in l_{p}$. Temos que $P_{m} \in \mathcal{P}_{f}\left({ }^{N} l_{p}, l_{p}\right)$ pois $P_{m}=\sum_{i=1}^{m} \phi_{i}^{N} \cdot\left(\lambda_{i} e_{i}\right) \operatorname{com} \phi_{i}: l_{p} \longrightarrow \mathbb{K}$ dado por $\phi_{i}(x)=x_{i}$ para cada $x=\left(x_{i}\right)_{i} \in l_{p}$.Sendo $\left(l_{p}, \odot,\|\| \cdot \| \mid\right)$ uma álgebra de Banach com identidade, pelas proposições 2.1 e 2.2 de [28] segue que $P_{m} \in \mathbb{P}_{f}\left({ }^{N} l_{p} ; l_{p}\right)$.

Seja $x \in l_{p} \operatorname{com}\|x\|_{p} \leq 1$. Para cada $m \in \mathbb{N}$

$$
\left\|P_{m}(x)-P(x)\right\|_{p}=\left\|\sum_{k=m+1}^{\infty} \lambda_{k} x_{k}^{N} e_{k}\right\|_{p}=\left(\sum_{k=m+1}^{\infty}\left|\lambda_{k}\right|^{p}\left|x_{k}^{N}\right|^{p}\right)^{\frac{1}{p}} \leq\left(\sum_{k=m+1}^{\infty}\left|\lambda_{k}\right|^{p}\right)^{\frac{1}{p}} .
$$

Como $\left(\lambda_{k}\right)_{k} \in l_{p}$, dado $\epsilon>0$ existe $m_{0} \in \mathbb{N}$ tal que $\left(\sum_{k=m+1}^{\infty}\left|\lambda_{k}\right|^{p}\right)^{\frac{1}{p}}<\epsilon \forall m \geq m_{0}$. Desta maneira, $P_{m} \stackrel{m \rightarrow \infty}{\longrightarrow} P$, ou seja, $P \in \overline{\mathcal{P}_{f}\left({ }^{N} l_{p} ; l_{p}\right)}\|\cdot\|$, e em particular, $P \in \overline{\mathbb{P}_{f}\left({ }^{N} l_{p} ; l_{p}\right)}\|\cdot\|$. E assim, temos que $C(P)=l_{p}$.

(2) Para a base canônica de $l_{p}, e_{n} \stackrel{\omega}{\longrightarrow} 0$ e para cada $n \in \mathbb{N}, P\left(e_{n}\right)=\lambda_{n} e_{n}$. Se $\left(\lambda_{k}\right)_{k} \notin c_{0}$ temos que $P\left(e_{n}\right) \nrightarrow 0$, e pela proposição 3.1.2 segue que $C(P)=\emptyset$. Sendo $l_{1}$ um espaço de Schur, temos que $P \notin \overline{\mathbb{P}_{f}\left({ }^{N} l_{p} ; l_{1}\right)}\|\cdot\|$. Agora, pela proposição 2.5 de [28] segue que $P \notin \overline{\mathcal{P}_{f}\left({ }^{N} l_{p} ; l_{1}\right)}\|\cdot\|$.

Agora vamos analisar o que ocorre quando $\left(\lambda_{k}\right)_{k} \in l_{1}$. Para cada $m \in \mathbb{N}$, tomemos $P_{m}: l_{p} \longrightarrow l_{1}$ dado por $P_{m}(x)=\sum_{i=1}^{m} \lambda_{i} x_{i}^{N} e_{i}$ para cada $x=\left(x_{i}\right)_{i} \in l_{p}$. Temos que $P_{m} \in \mathcal{P}_{f}\left({ }^{N} l_{p}, l_{1}\right)$ pois $P_{m}=\sum_{i=1}^{m} \phi_{i}^{N}\left(\lambda_{i} e_{i}\right) \operatorname{com} \phi_{i}: l_{p} \longrightarrow \mathbb{K}$ dado por $\phi_{i}(x)=x_{i}$ para cada $x=\left(x_{i}\right)_{i} \in l_{p}$.

Seja $x \in l_{p} \operatorname{com}\|x\|_{p} \leq 1$. Para cada $m \in \mathbb{N}$

$$
\left\|P_{m}(x)-P(x)\right\|_{1}=\left\|\sum_{k=m+1}^{\infty} \lambda_{k} x_{k}^{N} e_{k}\right\|_{1}=\sum_{k=m+1}^{\infty}\left|\lambda_{k} \| x_{k}^{N}\right| \leq \sum_{k=m+1}^{\infty}\left|\lambda_{k}\right| .
$$

Como $\left(\lambda_{k}\right)_{k} \in l_{1}$, dado $\epsilon>0$ existe $m_{0} \in \mathbb{N}$ tal que $\sum_{k=m+1}^{\infty}\left|\lambda_{k}\right|<\epsilon \forall m \geq m_{0}$. Assim temos que $P_{m} \stackrel{m \rightarrow \infty}{\longrightarrow} P$, ou seja, $P \in \overline{\mathcal{P}_{f}\left({ }^{N} l_{p} ; l_{1}\right)}\|\cdot\|$. E assim, temos que $C(P)=l_{p}$. Também, pela proposição 2.5 de [28] segue que $P \in \overline{\mathbb{P}_{f}\left({ }^{N} l_{p} ; l_{1}\right)}\|\cdot\|$.

Observamos que usando a técnica do exemplo acima, em um espaço de Banach $E$ com uma base de Schauder incondicional e normalizada $\left(x_{n}\right)_{n}$, dados $\left(\lambda_{k}\right)_{k} \in l_{1} \backslash c_{00}$ e 
$N \in \mathbb{N}$, para o polinômio $P: E \longrightarrow E$ definido por $P(x)=\sum_{k=1}^{\infty} \lambda_{k} \alpha_{k}^{N} x_{k}$ para cada $x=\sum_{k=1}^{\infty} \alpha_{k} x_{k} \in E$ temos que $C(P)=E$. Se além disso, $E$ é uma álgebra de Banach complexa com identidade, segue $P \in \overline{\mathbb{P}_{f}\left({ }^{N} E ; E\right)} \|^{\|\cdot\|}$.

\subsection{O conjunto $C_{\omega^{\star}}(P)$}

Nesta seção vamos considerar $E$ um espaço dual, ou seja, existe um espaço de Banach $G$ tal que $E=G^{\prime}$.

Dados $E, F$ espaços de Banach com $E$ espaço dual, aqui o nosso interesse é estudar o espaço vetorial formado pelos $P \in \mathcal{P}\left({ }^{N} E ; F\right)$ que são $\omega^{\star}$ contínuos sobre os limitados. Tal espaço vamos denotar por $\mathcal{P}_{w^{\star} b}\left({ }^{N} E ; F\right)$. Quando $F=\mathbb{K}, \mathcal{P}_{w^{\star} b}\left({ }^{N} E ; F\right)=\mathcal{P}_{w^{\star} b}\left({ }^{N} E\right)$. Vamos denotar por $\mathcal{P}_{w^{\star} b(0)}\left({ }^{N} E ; F\right)$ o espaço vetorial formado pelos $P \in \mathcal{P}\left({ }^{N} E ; F\right)$ que são $\omega^{\star}$ contínuos sobre os limitados que contém a origem.

Com uma argumentação simples, temos:

Proposição 3.2.1. 1. $P \in \mathcal{P}_{w^{\star} b}\left({ }^{N} E ; F\right)$ se e somente se $\left.P\right|_{B_{E}}$ é $\omega^{\star}$-contínuo.

2. $\mathcal{P}_{w^{\star} b}\left({ }^{N} E ; F\right)$ com a norma induzida de $\mathcal{P}\left({ }^{N} E ; F\right)$ é completo.

É bem conhecido, usando a bilinearidade, que para qualquer espaço de Banach $E$ valem $\mathcal{P}_{w b}\left({ }^{2} E\right)=\mathcal{P}_{w b(0)}\left({ }^{2} E\right)$ e $\mathcal{P}_{w s c}\left({ }^{2} E\right)=\mathcal{P}_{w s c(0)}\left({ }^{2} E\right)$. Já o mesmo não ocorre na topologia $\omega^{\star}$, como podemos ver no seguinte exemplo:

Exemplo 3.2.2. Seja $P: l_{1} \longrightarrow \mathbb{K}$ definido por $P(x)=x_{1} \sum_{j=1}^{\infty} x_{j}$ para cada $x=\left(x_{i}\right)_{i} \in l_{1}$. Então $P \in \mathcal{P}_{w^{\star} b(0)}\left({ }^{2} l_{1}\right) \backslash \mathcal{P}_{w^{\star} b}\left({ }^{2} l_{1}\right)$. Em particular, $P \in \mathcal{P}_{w^{\star} s c(0)}\left({ }^{2} l_{1}\right) \backslash \mathcal{P}_{w^{\star} s c}\left({ }^{2} l_{1}\right)$.

De fato: Para a seqüência $\left(e_{n}\right)_{n}$ temos que $e_{n}+e_{1} \stackrel{\omega^{\star}}{\longrightarrow} e_{1}$ e para cada $n \in \mathbb{N}, n \geq 2$, $P\left(e_{n}+e_{1}\right)=2$ e $P\left(e_{1}\right)=1$. Assim $P \notin \mathcal{P}_{w^{\star} b}\left({ }^{2} l_{1}\right)$. Por outro lado, seja $\left(f_{\alpha}\right)_{\alpha \in I}$ uma rede limitada em $l_{1}$ tal que $f_{\alpha} \stackrel{\omega^{\star}}{\longrightarrow} 0$. Para cada $\alpha \in I, f_{\alpha}=\left(f_{\alpha i}\right)_{i \in \mathbb{N}}$ e $f_{\alpha i} \stackrel{\alpha \in I}{\longrightarrow} 0$ para cada $i \in \mathbb{N}$. Para cada $\alpha \in I,\left|P\left(f_{\alpha}\right)-P(0)\right|=\left|f_{\alpha 1} \sum_{j=1}^{\infty} f_{\alpha j}\right| \leq\left|f_{\alpha 1}\right|\left\|f_{\alpha}\right\|_{1}$. Como $\left(f_{\alpha}\right)_{\alpha \in I}$ 
é uma rede limitada e $f_{\alpha 1} \stackrel{\alpha \in I}{\longrightarrow} 0$ segue que $P\left(f_{\alpha}\right) \stackrel{\alpha \in I}{\longrightarrow} P(0)$. Desta maneira, temos que $P \in \mathcal{P}_{w^{\star} b(0)}\left({ }^{2} l_{1}\right) \backslash \mathcal{P}_{w^{\star} b}\left({ }^{2} l_{1}\right)$.

Dados $N \in \mathbb{N}$ e $P \in \mathcal{P}\left({ }^{N} E ; F\right)$ é interessante estudar os seguintes conjuntos: $C_{w^{\star}}(P)=\left\{f \in E:\left.P\right|_{W}\right.$ é $\omega^{\star}$-contínuo $\forall W \subset E$ limitado com $\left.f \in W\right\}$ $C_{\star}(P)=\left\{f \in E: P\right.$ é $\omega^{\star}$-seqüencialmente contínuo em $\left.f\right\}$ e relacioná-los com os conjuntos: $C_{w}(P)=\left\{f \in E:\left.P\right|_{W}\right.$ é $\omega$-contínuo $\forall W \subset E$ limitado com $\left.f \in W\right\}$ $C(P)=\{f \in E: P$ é $\omega$-seqüencialmente contínuo em $f\}$.

Assim como na proposição 3.1.2, as seguintes afirmações valem:

Observações 3.2.3. $\quad$ i) $C_{w^{\star}}(P)$ é um subconjunto fechado de $E$.

ii) Se $f \in C_{w^{\star}}(P)$ então $\lambda f \in C_{w^{\star}}(P)$ para todo $\lambda \in \mathbb{K}$. Em particular, se $C_{w^{\star}}(P) \neq \emptyset$ então $0 \in C_{w^{\star}}(P)$.

iii) Também temos que $C_{w^{\star}}(P) \subseteq C_{\star}(P) \subseteq C(P)$ e $C_{w^{\star}}(P) \subseteq C_{w}(P) \subseteq C(P)$ pois $\omega^{\star} \leq \omega \leq\|\cdot\|$.

As observações (i) e (ii) seguem valendo para os conjuntos $C_{\star}(P)$ ou $C_{w}(P)$.

Exemplos 3.2.4. Para o polinômio $P: l_{1} \longrightarrow \mathbb{K}$ dado por $P(x)=x_{1} \sum_{j=1}^{\infty} x_{j}$ para cada $x=\left(x_{i}\right)_{i} \in l_{1}$, temos que $C_{w^{\star}}(P)=C_{\star}(P)=C_{w}(P)=\left\{x=\left(x_{i}\right)_{i} \in l_{1}: x_{1}=0\right\}$.

De fato: Vamos mostrar que $C_{\omega^{\star}}(P)=\left\{x=\left(x_{i}\right)_{i} \in l_{1}: x_{1}=0\right\}$. Seja $x=\left(x_{i}\right)_{i} \in l_{1}$ tal que $x_{1}=0$. Seja $\left(x_{\alpha}\right)_{\alpha \in I}$ uma rede limitada em $l_{1}$ tal que $x_{\alpha} \stackrel{\omega^{\star}}{\longrightarrow} x$. Para cada $\alpha \in I$, $x_{\alpha}=\left(x_{\alpha i}\right)_{i}$, e assim $x_{\alpha i} \stackrel{\alpha \in I}{\longrightarrow} x_{i}$ para cada $i \in \mathbb{N}$ e

$$
\left|P\left(x_{\alpha}\right)-P(x)\right|=\left|x_{\alpha 1} \sum_{j=1}^{\infty} x_{\alpha j}\right| \leq\left|x_{\alpha 1}\right|\left\|x_{\alpha}\right\|_{1} .
$$

Sendo $\left(x_{\alpha}\right)_{\alpha \in I}$ uma rede limitada e $x_{\alpha 1} \stackrel{\alpha \in I}{\longrightarrow} 0$, segue que $P\left(x_{\alpha}\right) \stackrel{\alpha \in I}{\longrightarrow} P(x)$.

Seja $x=\left(x_{i}\right)_{i} \in l_{1}$ tal que $x_{1} \neq 0$. Para a seqüência $\left(e_{n}\right)_{n}$ temos que $e_{n}+x \stackrel{\omega^{\star}}{\longrightarrow} x$ e para cada 
$n \geq 2,\left|P\left(e_{n}+x\right)-P(x)\right|=\left|x_{1}\right|>0$. Desta maneira, $C_{\omega^{\star}}(P)=\left\{x=\left(x_{i}\right)_{i} \in l_{1}: x_{1}=0\right\}$. De modo análogo, segue que $C_{\star}(P)=\left\{x=\left(x_{i}\right)_{i} \in l_{1}: x_{1}=0\right\}$. Observamos que $\frac{1}{n} e_{n}+x \stackrel{\omega}{\longrightarrow} x$ e $\left|P\left(\frac{1}{n} e_{n}+x\right)-P(x)\right|=\left|x_{1}\right|$ para cada $n \geq 2$, e assim, de modo análogo, temos que $C_{w}=\left\{x=\left(x_{i}\right)_{i} \in l_{1}: x_{1}=0\right\}$.

Observamos ainda que se $E=G^{\prime}$ onde $G$ é um espaço de Grothendieck segue que para cada espaço de Banach $F$, para cada $N \in \mathbb{N}$ e cada $P \in \mathcal{P}\left({ }^{N} E ; F\right)$ temos que $C_{\star}(P)=C(P)$, uma vez que $f_{n} \stackrel{\omega^{\star}}{\longrightarrow} f$ em $E$ implica que $f_{n} \stackrel{\omega}{\longrightarrow} f$ em $E$.

Para determinados espaços de Banach $E$, a caracterização dos polinômios $P \in \mathcal{P}\left({ }^{N} E\right)$ tais que $C_{w^{\star}}(P)=E$ é estudada por vários autores, dentre eles, citamos [3, 27, 34].

Assim como na proposição 3.1.2(3), dado um polinômio $N$-homogêneo contínuo $P: E \longrightarrow F$ podemos caracterizar os subconjuntos:

$$
\begin{aligned}
& C_{w^{\star}}(P)=\cap_{j=0}^{N-1}\left\{f \in E: \Phi_{j}(f) \in \mathcal{P}_{w^{\star} b(0)}\left({ }^{N-j} E ; F\right)\right\} \\
& C_{\star}(P)=\cap_{j=0}^{N-1}\left\{f \in E: \Phi_{j}(f) \in \mathcal{P}_{w^{\star} s c(0)}\left({ }^{N-j} E ; F\right)\right\} \\
& C_{w}(P)=\cap_{j=0}^{N-1}\left\{f \in E: \Phi_{j}(f) \in \mathcal{P}_{w b(0)}\left({ }^{N-j} E ; F\right)\right\} .
\end{aligned}
$$

Dados $N \in \mathbb{N}, N \geq 2$, e $P \in \mathcal{P}\left({ }^{N} E ; F\right)$, seja $A$ a aplicação $N$-linear simétrica associada ao polinômio $P$. O operador associado $T_{P}$, ou seja, $T_{P}: E \longrightarrow \mathcal{P}\left({ }^{N-1} E ; F\right)$ é definido por $T_{P}(f)(g)=A\left(f, g^{N-1}\right)$ para cada $f, g \in E$. E seja $d P$, a aplicação derivada de $P$, ou seja, $d P: E \longrightarrow \mathcal{L}(E ; F)$ é dada por $d P(f)(g)=N \cdot A\left(f^{N-1}, g\right)$ para cada $f, g \in E$. Sendo $A$ simétrica, segue que $d P(f)(g)=N \cdot T_{P}(g)(f)$.

Aron, Hérves e Valdivia em [7], teorema 2.9, demonstram que $P \in \mathcal{P}_{w b}\left({ }^{N} E ; F\right)$ se e somente se o seu operador associado $T_{P}$ é compacto. Carando e Dimant em [13], lema 3.1, mostram que se $P \in \mathcal{P}_{w^{\star} b}\left({ }^{N} E ; F\right)$ então o operador associado $T_{P} \in \mathcal{L}_{w^{\star} b}\left(E ; \mathcal{P}_{w^{\star} b}\left({ }^{N-1} E ; F\right)\right)$. Em geral, não vale a recíproca, mesmo quando $F=\mathbb{C}$, como podemos ver no seguinte exemplo: 
Exemplo 3.2.5. Seja $P: l_{1} \longrightarrow \mathbb{K}$ dado por $P(x)=\sum_{i=1}^{\infty} x_{i}^{2}$ para cada $x=\left(x_{i}\right)_{i} \in l_{1}$. Temos que $C_{w^{\star}}(P)=\emptyset$ e $T_{P} \in \mathcal{L}_{w^{\star} b}\left(l_{1} ; c_{0}\right)$.

De fato: Para a seqüência $\left(e_{n}\right)_{n}$ temos que $e_{n} \stackrel{\omega^{\star}}{\longrightarrow} 0$ e $P\left(e_{n}\right)=1$ para cada $n \in \mathbb{N}$, mas $P(0)=0$, e assim, $C_{w^{\star}}(P)=\emptyset$. Agora, $T_{P}: l_{1} \longrightarrow l_{\infty}$ é dada por $T_{P}(x)(y)=\sum_{i=1}^{\infty} x_{i} y_{i}$ para cada $x=\left(x_{i}\right)_{i}, y=\left(y_{i}\right)_{i} \in l_{1}$.

O operador $T_{P}$ nada mais é que o adjunto de $S: l_{1} \longrightarrow c_{0}$ dado por $S\left(\left(x_{i}\right)_{i}\right)=\left(x_{i}\right)_{i}$. Como $S$ é contínuo, segue de [30], teorema 3.1.11, que $S^{*}=T_{P}$ é $\omega^{\star}-\omega^{\star}$-contínuo. Desta maneira, para cada $x \in l_{1}$ temos que $T_{P}(x) \in c_{0}$, ou seja, $T_{P} \in \mathcal{L}_{\omega^{\star} b}\left(l_{1} ; c_{0}\right)$.

Proposição 3.2.6. Sejam $N \in \mathbb{N}, N \geq 2$, e $P \in \mathcal{P}\left({ }^{N} E ; F\right)$ tal que $C_{w^{\star}}(P) \neq \emptyset$. Então, para cada $f \in C_{w^{\star}}(P)$ temos que $C_{\omega^{\star}}(d P(f))=E$.

Demonstração: Como $C_{w^{\star}}(P)=\cap_{j=0}^{N-1}\left\{f \in E: \Phi_{j}(f) \in \mathcal{P}_{w^{\star} b(0)}\left({ }^{N-j} E ; F\right)\right\}$, dado $f \in C_{w^{\star}}(P)$, observamos que: i) $f \in C_{\omega^{\star}}\left(\Phi_{N-1}(f)\right)$ e ii) $f \in C_{\omega^{\star}}(d P(f))$. De fato: i) Seja $\left(f_{\alpha}\right)_{\alpha \in I}$ uma rede limitada em $E$ tal que $f_{\alpha} \stackrel{\omega^{\star}}{\longrightarrow} f$. Pela fórmula da polarização, $\Phi_{N-1}(f)\left(f_{\alpha}\right)=A\left(f^{N-1}, f_{\alpha}\right)=\frac{1}{2^{N} N !} \sum_{\epsilon_{i}= \pm 1} \epsilon_{1} \cdot \ldots \cdot \epsilon_{N} P\left(\left(\sum_{i=1}^{N-1} \epsilon_{i}\right) f+\epsilon_{N} f_{\alpha}\right)$.

$\operatorname{Mas}\left(\left(\sum_{i=1}^{N-1} \epsilon_{i}\right) f+\epsilon_{N} f_{\alpha}\right)_{\alpha} \stackrel{\omega^{\star}}{\longrightarrow}\left(\sum_{i=1}^{N} \epsilon_{i}\right) f$ e $\left(\sum_{i=1}^{N} \epsilon_{i}\right) f \in C_{\omega^{\star}}(P)$. E assim, $\lim _{\alpha \in I} \Phi_{N-1}(f)\left(f_{\alpha}\right)=\Phi_{N-1}(f)(f)$.

ii) Seja $\left(g_{\alpha}\right)_{\alpha \in I}$ uma rede limitada em $E$ tal que $g_{\alpha} \stackrel{\omega^{\star}}{\longrightarrow} f$. Por (i) $\lim _{\alpha \in I} \Phi_{N-1}(f)\left(g_{\alpha}\right)=$ $\Phi_{N-1}(f)(f)$. Mas

$$
\begin{gathered}
\Phi_{N-1}(f)\left(g_{\alpha}\right)=A\left(f^{N-1}, g_{\alpha}\right)=\frac{1}{N} d P(f)\left(g_{\alpha}\right) \\
\Phi_{N-1}(f)(f)=A\left(f^{N-1}, f\right)=\frac{1}{N} d P(f)(f) .
\end{gathered}
$$

E assim, segue que $\lim _{\alpha \in I} d P(f)\left(g_{k}\right)=d P(f)(f)$.

Agora vamos mostrar que $C_{\omega^{\star}}(d P(f))=E$. Sejam $g \in E$ e $\left(f_{\alpha}\right)_{\alpha \in I}$ uma rede limitada em $E$ tal que $f_{\alpha} \stackrel{\omega^{\star}}{\longrightarrow} g$. Assim, $f_{\alpha}-g \stackrel{\omega^{\star}}{\longrightarrow} 0$ e conseqüentemente $\lim _{\alpha \in I} d P(f)\left(f_{\alpha}-g\right)=d P(f)(0)$ pois $0 \in C_{\omega^{\star}}(d P(f))$. A aplicação $d P(f)$ é um operador linear, logo $d P(f)\left(f_{\alpha}-g\right)=$ $d P(f)\left(f_{\alpha}\right)-d P(f)(g)$. Também temos que $d P(f)(0)=N A\left(f^{N-1}, 0\right)=0$. Portanto $\lim _{\alpha \in I} d P(f)\left(f_{\alpha}\right)=d P(f)(g)$. 
Observação 3.2.7. Resultado análogo também vale para a topologia $\omega$.

Segue como corolário o seguinte resultado, também demonstrado por Moraes e Llavona em [27], proposição 1.7. Lembramos que um espaço de Banach $E$ tem a propriedade de Mazur se cada $f \in E^{\prime \prime} \omega^{\star}$-seqüencialmente contínuo é $\omega^{\star}$ contínuo, ou seja, $f \in E$.

Corolário 3.2.8. Seja $E=G^{\prime}$ com $G$ um espaço de Banach e suponhamos que $G$ tem a propriedade de Mazur. Então para cada $N \in \mathbb{N}, N \geq 2$, e cada $P \in \mathcal{P}\left({ }^{N} E\right)$ com $C_{w^{\star}}(P)=E$ tem-se que $d P(f)$ é $\omega^{\star}$ contínuo para cada $f \in E$.

Demonstração: Para cada $f \in E$, pela proposição 3.2.6 e observação 3.2.3(iii) temos que $C_{\star}(d P(f))=E$. Como $G$ tem a propriedade de Mazur, segue que $d P(f)$ é $\omega^{\star}$-contínuo para cada $f \in E$.

Observação 3.2.9. Sejam $E=G^{\prime}$ com $G$ um espaço de Banach, $N \in \mathbb{N}, N \geq 2$, e $P \in \mathcal{P}\left({ }^{N} E\right)$. Se $T_{P} \in \mathcal{L}_{w^{\star} b}\left(E ; \mathcal{P}_{w^{\star} b}\left({ }^{N-1} E\right)\right)$ e para cada $x \in E, d P(x) \in G$ então $P \in \mathcal{P}_{w^{\star} b}\left({ }^{N} E\right)$. Reciprocamente, se $G$ tem a propriedade de Mazur, $P \in \mathcal{P}_{w^{\star} b}\left({ }^{N} E\right)$ implica que $T_{P} \in \mathcal{L}_{w^{\star} b}\left(E ; \mathcal{P}_{w^{\star} b}\left({ }^{N-1} E\right)\right)$ e para cada $x \in E, d P(x) \in G$.

De fato: Seja $P \in \mathcal{P}\left({ }^{N} E\right)$ tal que $T_{P} \in \mathcal{L}_{w^{\star} b}\left(E ; \mathcal{P}_{w^{\star} b}\left({ }^{N-1} E\right)\right)$ e para cada $x \in E$, $d P(x) \in G$. Pelo teorema 1.5 de $[27]$ basta mostrar que $P \in \mathcal{P}_{w b}\left({ }^{N} E\right)$. Como $T_{P} \in$ $\mathcal{L}_{w^{\star} b}\left(E ; \mathcal{P}_{w^{\star} b}\left({ }^{N-1} E\right)\right)$, temos que $T_{P} \in \mathcal{L}_{w b}\left(E ; \mathcal{P}\left({ }^{N-1} E\right)\right)$ e assim, do lema 2.2 e proposição 2.5 de [8], $T_{P}$ é compacto. Agora do teorema 2.9 de [7] segue que $P \in \mathcal{P}_{w b}\left({ }^{N} E\right)$. A recíproca segue do lema 3.1 de [13] e corolário 3.2.8.

Observação 3.2.10. Sejam $N \in \mathbb{N}, P \in \mathcal{P}\left({ }^{N} E\right)$ e $f \in E$. Suponhamos que cada rede limitada $\left(f_{\alpha}\right)_{\alpha \in I}$ em $E$ com $f_{\alpha} \stackrel{\omega^{\star}}{\longrightarrow} f$ admite uma subrede $\left(f_{\beta}\right)_{\beta \in J}$ tal que $P\left(f_{\beta}\right) \longrightarrow P(f)$. Então $f \in C_{w^{\star}}(P)$.

De fato: Suponhamos que $f$ não pertença a $C_{w^{\star}}(P)$. Então existe uma rede limitada $\left(f_{\alpha}\right)_{\alpha \in I}$ em $E$ tal que $f_{\alpha} \stackrel{\omega^{\star}}{\longrightarrow} f$ e $P\left(f_{\alpha}\right)$ não converge para $P(f)$. Assim existem $\epsilon>0$ e uma subrede $\left(f_{\beta}\right)_{\beta \in J}$ de $\left(f_{\alpha}\right)_{\alpha \in I}$ tal que $\left|P\left(f_{\beta}\right)-P(f)\right|>\epsilon$ para todo $\beta \in J$. Como 
$f_{\beta} \stackrel{\omega^{\star}}{\longrightarrow} f$, por hipótese, $\left(f_{\beta}\right)_{\beta \in J}$ admite uma subrede $\left(f_{\gamma}\right)_{\gamma \in J_{1}}$ tal que $P\left(f_{\gamma}\right) \stackrel{\gamma \in J_{1}}{\longrightarrow} P(f)$, uma contradição.

Observamos que resultado análogo também vale para os conjuntos $C_{\star}(P), C_{w}(P)$ e $C(P)$.

Sejam $\left(x_{\alpha}\right)_{\alpha \in I}$ e $\left(y_{\beta}\right)_{\beta \in J}$ duas redes e seja $K=I \times J \operatorname{com}\left(\alpha_{1}, \beta_{1}\right) \preceq\left(\alpha_{2}, \beta_{2}\right)$ quando $\alpha_{1} \preceq \alpha_{2}$ e $\beta_{1} \preceq \beta_{2}$. Tomando $g: K \longrightarrow I$ e $h: K \longrightarrow J$ as aplicações projeção, ou seja, $g(\alpha, \beta)=\alpha$ e $h(\alpha, \beta)=\beta$, temos que $\left(x_{g(\alpha, \beta)}\right)_{(\alpha, \beta) \in K}$ e $\left(y_{h(\alpha, \beta)}\right)_{(\alpha, \beta) \in K}$ são subredes de $\left(x_{\alpha}\right)_{\alpha \in I}$ e $\left(y_{\beta}\right)_{\beta \in J}$ tendo o mesmo conjunto de índices. Se $\left(x_{\alpha}\right)_{\alpha \in I}$ pertence a algum espaço topológico, então $\left(x_{g(\alpha, \beta)}\right)_{(\alpha, \beta) \in K}$ converge para algum $x$ se e somente se $\left(x_{\alpha}\right)_{\alpha \in I}$ converge a $x$, e analogamente para $\left(y_{h(\alpha, \beta)}\right)_{(\alpha, \beta) \in K}$ e $\left(y_{\beta}\right)_{\beta \in J}$.

Com argumentação análoga de [5] e usando os fatos acima mencionados temos:

Proposição 3.2.11. Sejam $P \in \mathcal{P}\left({ }^{N} E\right)$ e $Q \in \mathcal{P}\left({ }^{M} E\right)$. Então

$$
C_{w^{\star}}(P \cdot Q)=\left(C_{w^{\star}}(P) \cap C_{w^{\star}}(Q)\right) \cup\left(C_{w^{\star}}(P) \cap P^{-1}(0)\right) \cup\left(C_{w^{\star}}(Q) \cap Q^{-1}(0)\right) .
$$

Seja $E=G^{\prime} \operatorname{com} G$ um espaço de Banach. Observemos os seguintes espaços especiais: $\mathcal{P}_{f^{\star}}\left({ }^{N} E\right)=\left\{P \in \mathcal{P}\left({ }^{N} E\right): P=\sum_{j=1}^{q} x_{1 j} \cdot \ldots \cdot x_{N j} \quad x_{l i} \in G\right\}$ $\mathcal{P}_{f}\left({ }^{N} E\right)=\left\{P \in \mathcal{P}\left({ }^{N} E\right): P=\sum_{j=1}^{s} \psi_{1 j} \cdot \ldots \cdot \psi_{N j} \quad \psi_{l i} \in E^{\prime}\right\}$ $\mathcal{P}_{c^{\star}}\left({ }^{N} E\right)=\overline{\mathcal{P}}_{f^{\star}\left({ }^{N} E\right)}\|\cdot\|$ e $\mathcal{P}_{w^{\star} s c}\left({ }^{N} E\right)=\left\{P \in \mathcal{P}\left({ }^{N} E\right): P\right.$ é $\omega^{\star}$ - seqüencialmente contínuo $\}$. Do fato de $\omega^{\star} \leq \omega \leq\|\cdot\|$ seguem as seguintes inclusões:

i) $\mathcal{P}_{f^{\star}}\left({ }^{N} E\right) \subset \mathcal{P}_{w^{\star} b}\left({ }^{N} E\right) \subset \mathcal{P}_{w^{\star} s c}\left({ }^{N} E\right) \subset \mathcal{P}_{w s c}\left({ }^{N} E\right)$.

ii) $\mathcal{P}_{c^{\star}}\left({ }^{N} E\right) \subset \mathcal{P}_{w^{\star} b}\left({ }^{N} E\right) \subset \mathcal{P}_{w b}\left({ }^{N} E\right)$.

Em [5], utilizando-se da fórmula $C(P \cdot Q)$, Aron e Dimant obtêm algumas conseqüências interessantes sobre a irredutibilidade dos polinômios homogêneos contínuos. Aqui, a fórmula dada pela proposição 3.2.11 nos dará algumas aplicações sobre as extensões de Aron-Berner de polinômios homogêneos contínuos. Dizemos que um polinômio 
$\bar{P} \in \mathcal{P}\left({ }^{N} E^{\prime \prime}\right)$ é uma extensão de Aron-Berner de algum polinômio $P \in \mathcal{P}\left({ }^{N} E\right)$ se $\bar{P}$ é a extensão de $P$ para $E^{\prime \prime}$ que preserva a norma.

Exemplos 3.2.12. Sejam $N \in \mathbb{N}$ com $N \geq 2 e \varphi \in l_{\infty}^{\prime}$. Tomemos $P: l_{\infty} \longrightarrow \mathbb{K}$ dado por $P(x)=\left(x_{1}-x_{2}\right)^{N-1} \cdot \varphi(x)$ para cada $x=\left(x_{i}\right)_{i} \in l_{\infty}$.

1. Se $\varphi \in l_{\infty}^{\prime} \backslash l_{1}$ então $P$ não é extensão de Aron-Berner de nenhum polinômio $N$ homogêneo em $c_{0}$.

2. Se $\varphi \in l_{1}, P$ é extensão de Aron-Berner de algum polinômio $N$-homogêneo em $c_{0}$.

De fato: $P(x)=P_{1} \cdot \varphi(x)$ onde $P_{1}(x)=\left(x_{1}-x_{2}\right)^{N-1}$. Pela proposição 3.2.11 temos que $C_{w^{\star}}(P)=\left(C_{w^{\star}}\left(P_{1}\right) \cap C_{w^{\star}}(\varphi)\right) \cup\left(C_{w^{\star}}\left(P_{1}\right) \cap P_{1}^{-1}(0)\right) \cup\left(C_{w^{\star}}(\varphi) \cap \varphi^{-1}(0)\right)$. Se $\varphi \in l_{\infty}^{\prime} \backslash l_{1}, C_{w^{\star}}(P)=P_{1}^{-1}(0)$ pois $C_{w^{\star}}\left(P_{1}\right)=l_{\infty}$, e $C_{w^{\star}}(\varphi)=\emptyset$. Desta maneira, $C_{w^{\star}}(P)=\left\{x=\left(x_{i}\right)_{i} \in l_{\infty}: x_{1}=x_{2}\right\}$. Assim, segue de [3] que $P$ não é extensão de Aron-Berner de nenhum polinômio $N$-homogêneo contínuo em $c_{0}$.

Se $\varphi \in l_{1}, C_{w^{\star}}(P)=l_{\infty}$, pois $P \in \mathcal{P}_{f^{\star}}\left({ }^{N} l_{\infty}\right)$. E assim, segue de [3] que $P$ é extensão de Aron-Berner de algum polinômio $N$-homogêneo contínuo em $c_{0}$. 


\section{Capítulo 4}

\section{Zeros de polinômios}

\subsection{Introdução}

Neste capítulo temos por objetivo apresentar uma melhor limitação para $m$, quando $d \geq 3$, tal que para cada polinômio $d$-homogêneo $P: \mathbb{C}^{m} \longrightarrow \mathbb{C}$ existe um subespaço vetorial $X_{P} \subset P^{-1}(0)$ de dimensão $n$. Os resultados aqui apresentados fazem parte do trabalho [29] aceito para publicação em Linear and Multilinear Algebra.

Com um simples argumento de indução Aron e Rueda em [9] demonstram o seguinte teorema:

Teorema 4.1.1 (Aron e Rueda, [9]). Existe uma função $\theta: \mathbb{N} \times \mathbb{N} \longrightarrow \mathbb{N}, \theta(n, d)=m$, com a seguinte propriedade: Para cada polinômio d-homogêneo $P: \mathbb{C}^{m} \longrightarrow \mathbb{C}$ existe um subespaço vetorial $X_{P} \subset P^{-1}(0)$ de dimensão $n$.

Em outras palavras, existe uma função $\phi: \mathbb{N} \times \mathbb{N} \longrightarrow \mathbb{N}_{0}, \phi(m, d)=n$, que satisfaz a propriedade acima e para cada $d \in \mathbb{N}, \lim _{m \rightarrow \infty} \phi(m, d)=\infty$.

Em [9] Aron e Rueda discutem o seguinte fato: É possível calcular explicitamente a melhor função $\theta$ ? Ou seja, encontar a melhor limitação sobre o número de variáveis $m$ em função dos naturais $n$ e $d$ que satisfaça o teorema 4.1.1. 
Dado $d \in \mathbb{N}$ fixo, vamos denotar por $\theta_{d}(n)$ o menor valor de $\theta(n, d)$ para cada $n \in \mathbb{N}$ que satisfaz o teorema 4.1.1. E por $\phi_{d}(m)$ o maior valor de $\phi(m, d)$ para cada $m \in \mathbb{N}$.

Observamos que no caso linear, basta tomar as funções $\theta_{1}: \mathbb{N} \longrightarrow \mathbb{N}$ e $\phi_{1}: \mathbb{N} \longrightarrow \mathbb{N}_{0}$ dadas por $\theta_{1}(n)=m:=n+1$ e $\phi_{1}(m)=n:=m-1$.

Aron e Rueda no artigo [9] obtiveram uma limitação para o número de variáveis $m$ em função da dimensão $n$ e da homogeneidade $d$ para $d=2,3$ e 4 . E no caso $d=2$ eles obtiveram a melhor limitação:

Proposição 4.1.2 (Aron and Rueda, [9]). A função $\theta_{2}: \mathbb{N} \longrightarrow \mathbb{N}$, definida por $\theta_{2}(n)=m:=2 n$ satisfaz: Para cada polinômio 2-homogêneo $P: \mathbb{C}^{m} \longrightarrow \mathbb{C}$ existe um subespaço $X_{P} \subset P^{-1}(0)$ de dimensão $n$. Além disso, para cada $n \in \mathbb{N}$, o valor $\theta_{2}(n)$ é a melhor limitação sobre o número de variáveis $m$.

E conseqüentemente, a função $\phi_{2}: \mathbb{N} \longrightarrow \mathbb{N}_{0}$ é definida por $\phi_{2}(m):=\left\lceil\frac{m}{2}\right\rceil$, onde $\left\lceil\frac{m}{2}\right\rceil$ é o maior inteiro menor ou igual a $\frac{m}{2}$.

Nos casos $d=3$ e $d=4$, as limitações sobre $m$ em função da homogeneidade $d$ e da dimensão $n$ encontradas por Aron e Rueda em [9] assumem valores muito grandes, no que segue vamos apresentar uma melhor limitação para tal $m$.

\subsection{Limitação para $d \geq 3$}

Nosso objetivo nesta seção é mostrar que existe uma melhor limitação sobre o número de variáveis $m$ em função de $n$ e da homogeneidade $d, d \geq 3$. Para isso, vamos utilizar fortemente os seguintes fatos:

$(\mathcal{P} 1)$ Para cada polinômio $d$-homogêneo $P: \mathbb{C}^{m} \longrightarrow \mathbb{C}$ com $m \geq 2$ existe $z_{1}$ não nulo em $\mathbb{C}^{m}$ tal que $P\left(z_{1}\right)=0$.

$(\mathcal{P} 2)$ Para cada polinômio $d$-homogêneo $P: \mathbb{C}^{m} \longrightarrow \mathbb{C}$ segue que $P\left(a_{1} z_{1}+\ldots+a_{s} z_{s}\right)=0$ com $a_{1}, \ldots, a_{s} \in \mathbb{C}$ se para cada s-upla $\left(m_{1}, \ldots, m_{s}\right) \in\left(\mathbb{N}_{0}\right)^{s} \operatorname{com} m_{1}+\ldots+m_{s}=d$ 
tem-se $A\left(z_{1}^{m_{1}}, \ldots, z_{s}^{m_{s}}\right)=0$, pois da fórmula de Leibniz, $P\left(a_{1} z_{1}+\ldots+a_{s} z_{s}\right)=\sum_{m_{1}+\ldots+m_{s}=d} \frac{d !}{m_{1} ! \ldots \cdot m_{s} !} a_{1}^{m_{1}} \cdot \ldots \cdot a_{s}^{m_{s}} A\left(z_{1}^{m_{1}}, \ldots, z_{s}^{m_{s}}\right)$, onde $A$ é a aplicação $d$-linear simétrica associada a $P$ e $A\left(z_{1}^{m_{1}}, \ldots, z_{s}^{m_{s}}\right)=A\left(z_{1}, . m_{1}, z_{1}, \ldots, z_{s}, m_{s}, z_{s}\right)$.

Para exibir a limitação, também vamos nos valer do seguinte resultado de análise combinatória. Primeiramente vamos relembrar alguns conceitos de combinatória. Denominamos combinações de $n$ elementos distintos tomados $r$ a $r$, onde $n \geq 1$ e $r$ é um número natural tal que $r \leq n$, aos conjuntos formados de $r$ elementos distintos escolhidos entre os $n$ elementos dados. Representamos pelo símbolo $C_{n}^{r}$ o número de combinações de $n$ elementos tomados $r$ a $r$ e é dada por $C_{n}^{r}:=\frac{n !}{r !(n-r) !}$.

Consideremos $n$ objetos distintos. Observamos que o número de maneiras de escolhermos $r$ objetos é idêntico ao número de maneiras de escolhermos $n-r$ objetos, pois se dos $n$ objetos tirarmos $r$, sobram $n-r$ e, conseqüentemente, se de $n$ objetos tirarmos $n-r$, sobram $r$. Logo $C_{n}^{r}=C_{n}^{n-r}$, onde $C_{n}^{n-r}$ é chamada de combinação complementar de $C_{n}^{r}$.

Lema 4.2.1. O número de soluções inteiras e não negativas para a equação $s_{1}+\ldots+s_{k}=p(\mathbf{I})$ com $k$ incógnitas é dada por:

$$
C_{k+p-1}^{p}:=\frac{(k+p-1) !}{p !(k-1) !} .
$$

Demonstração: Somando o valor um $(=1)$ a cada $s_{i}$, obtemos

$$
\left(s_{1}+1\right)+\ldots+\left(s_{k}+1\right)=p+k .
$$

Se chamarmos $s_{i}+1=y_{i}$, para cada $i=1, \ldots, k$, teremos

$$
y_{1}+\ldots+y_{k}=p+k, \text { com } y_{i} \geq 1
$$

Como a cada solução de inteiros e não negativos de (I) associamos uma solução de inteiros e positivos de (II) e vice-versa, então o número de soluções inteiras e não negativas da equação $s_{1}+\ldots+s_{k}=p$ é igual ao número de soluções inteiras e positivas para a equação $y_{1}+\ldots+y_{k}=p+k$. Como estamos interessados em expressar o inteiro positivo $(p+k)$ 
como soma de $k$ inteiros positivos, basta colocarmos $(k-1)$ barras divisoras entre os $(k+p) 1$ 's:

$$
1+1+|1+\ldots+1+| \ldots+\mid 1+\ldots+1=p+k .
$$

O valor de $y_{1}$ será o número de 1's que antecedem a primeira barra, o valor de $y_{2}$, o número de 1's entre a primeira e a segunda barra, e assim por diante, até obtermos o valor de $y_{k}$ como sendo o número de 1's à direita da barra de número $(k-1)$. Como a cada possível distribuição de barras corresponde uma única solução para a equação (II), basta contarmos de quantas formas isto pode ser feito. Devemos selecionar $(k-1)$ dos $(k+p-1)$ possíveis locais (os sinais de " + " que separam os 1's) para a colocação das barras divisoras, o que pode ser feito de $C_{k+p-1}^{k-1}$ maneiras. Como $C_{k+p-1}^{k-1}=C_{k+p-1}^{p}$ segue que o número de soluções inteiras e não negativas para a equação $s_{1}+\ldots+s_{k}=p$ é dado por $C_{k+p-1}^{p}:=\frac{(k+p-1) !}{p !(k-1) !}$.

Observação 4.2.2. Para cada polinômio d-homogêneo $P: \mathbb{C}^{m} \longrightarrow \mathbb{C}$, aplicando $(\mathcal{P} 1)$, podemos tomar $\theta_{d}(1)=2$.

Como mencionamos anteriormente, as limitações encontradas por Aron e Rueda em [9] para $\theta_{3}(n)$ e $\theta_{4}(n)$ assumem valores muito grandes.

Nosso objetivo agora é calcular uma melhor limitação para $\theta_{3}(n)$ e $\theta_{4}(n)$. Na próxima proposição vamos calcular a limitação $\theta_{3}(n)$. Para $n=1,2$ o valor da limitação aqui encontrada coincide com o valor apresentado por Aron e Rueda. Para $n \geq 3$, a limitação aqui encontrada assume valores substancialmente menores que os apresentados em [9].

Proposição 4.2.3. Para $d=3$, para cada $n \in \mathbb{N}$ temos que $\theta_{3}(n) \leq 2^{n}+\left(\frac{n^{2}+n}{2}\right)-1$.

Demonstração: A demonstração será feita por indução sobre $n$. Pela observação 4.2.2, podemos tomar $\theta_{3}(1)=2$. Suponhamos que por indução já calculamos as limitações para $\theta_{3}(1), \ldots, \theta_{3}(n-1)$ e digamos que $\theta_{3}(n-1) \leq M$ para algum $M \in \mathbb{N}$. Sejam $m \geq M+1$ (a ser determinado), $P: \mathbb{C}^{m} \longrightarrow \mathbb{C}$ um polinômio 3-homogêneo e $A: \mathbb{C}^{m} \times \mathbb{C}^{m} \times \mathbb{C}^{m} \longrightarrow \mathbb{C}$ a aplicação 3-linear simétrica associada a $P$. Como $m \geq M+1$ então existem $z_{1}, \ldots, z_{n-1} \in \mathbb{C}^{m}$ tais que $\left\{z_{1}, \ldots, z_{n-1}\right\}$ é linearmente independente e 
$\left[z_{1}, \ldots, z_{n-1}\right] \subset P^{-1}(0)$. Podemos escrever $\mathbb{C}^{m}=\left[z_{1}, \ldots, z_{n-1}\right] \oplus Y$, onde $\operatorname{dim} Y=m-n+1$, e existe $T_{1}: \mathbb{C}^{m-n+1} \longrightarrow Y$ um isomorfismo.

Seja $A_{1}: \mathbb{C}^{m-n+1} \longrightarrow \mathbb{C}$ a aplicação linear dada por $A_{1}(x)=A\left(z_{1}, z_{1}, T_{1}(x)\right)$ para cada $x \in \mathbb{C}^{m-n+1}$. Então $\operatorname{dimKer} A_{1}=m-n=\phi_{1}(m-n+1)$, se $A_{1}$ é não nula, se $A_{1} \equiv 0$ tomemos um subespaço $W_{1}$ de $\mathbb{C}^{m-n+1}$ de dimensão $(m-n)$.

Seja $X_{1}=T_{1}\left(A_{1}^{-1}(0)\right)$ (ou $X_{1}=T_{1}\left(W_{1}\right)$ se $\left.A_{1} \equiv 0\right)$. Então $X_{1} \subset Y$ e $\operatorname{dim} X_{1}=\phi_{1}(m-n+1)$. Logo existe $T_{2}: \mathbb{C}^{\phi_{1}(m-n+1)} \longrightarrow X_{1}$ um isomorfismo. Considere $A_{2}: \mathbb{C}^{\phi_{1}(m-n+1)} \longrightarrow \mathbb{C}$ a aplicação linear dada por $A_{2}(x)=A\left(z_{2}, z_{2}, T_{2}(x)\right)$ para cada $x \in \mathbb{C}^{\phi_{1}(m-n+1)}$. Então temos que $\operatorname{dimKer} A_{2}=\phi_{1}(m-n+1)-1=m-n-1=$ $\phi_{1}\left(\phi_{1}(m-n+1)\right)$ se $A_{2}$ for não nula. Caso contrário, tomemos um subespaço $W_{2}$ de $\mathbb{C}^{\phi_{1}(m-n+1)}$ de dimensão $(m-n-1)$.

Seja $X_{2}=T_{2}\left(A_{2}^{-1}(0)\right)$ (ou $X_{2}=T_{2}\left(W_{2}\right)$ se $A_{2} \equiv 0$ ). Então $X_{2} \subset X_{1}$ e $\operatorname{dim} X_{2}=\phi_{1}\left(\phi_{1}(m-n+1)\right)$. Logo existe $T_{3}: \mathbb{C}^{\phi_{1}\left(\phi_{1}(m-n+1)\right)} \longrightarrow X_{2}$ um isomorfismo.

Prosseguindo de maneira análoga para cada par $\left(z_{i}, z_{j}\right)$ com $1 \leq i \leq j \leq n-1$, construímos $k_{1}$ funcionais lineares e encontramos $Z_{1} \subset Y$ tal que $A\left(z_{i}^{s_{i}}, z_{j}^{s_{j}}, x\right)=0$ para cada $x \in Z_{1}$ e cada $(n-1)$-upla $\left(s_{1}, \ldots, s_{n-1}\right) \in\left(\mathbb{N}_{0}\right)^{n-1}$ com $s_{1}+\ldots+s_{n-1}=2$ onde $k_{1}$ é o número de soluções inteiras e não negativas da equação $s_{1}+\ldots+s_{n-1}=2$. Também, $\operatorname{dim} Z_{1}=m-n+1-k_{1}=\phi_{1}\left(\stackrel{\left(k_{1}\right)}{\cdots}\left(\phi_{1}(m-n+1) \ldots\right)\right)$.

Seja $L_{1}=\phi_{1}\left({ }^{\left(k_{1}\right)}\left(\phi_{1}(m-n+1) \ldots\right)\right)$. Suponhamos que o valor de $m$ que estamos determinando seja tal que $L_{1} \geq 2$. Então existe um isomorfismo $F_{1}: \mathbb{C}^{L_{1}} \longrightarrow Z_{1}$.

Agora seja o polinômio 2-homogêneo $Q_{1}: \mathbb{C}^{L_{1}} \longrightarrow \mathbb{C}$ dado por $Q_{1}(x)=A\left(z_{1}, F_{1}(x), F_{1}(x)\right)$ para cada $x \in \mathbb{C}^{L_{1}}$. Pela proposição 4.1 .2 existe um subespaço $X_{Q_{1}} \subset \mathbb{C}^{L_{1}}$ tal que $X_{Q_{1}} \subset Q_{1}^{-1}(0)$ e $\operatorname{dim} X_{Q_{1}}=\phi_{2}\left(L_{1}\right)=\left\lceil\frac{L_{1}}{2}\right\rceil$. Seja $Y_{1}=F_{1}\left(X_{Q_{1}}\right)$. Então $Y_{1} \subset Z_{1} \mathrm{e}$ $\operatorname{dim} Y_{1}=\phi_{2}\left(L_{1}\right)$, logo existe um isomorfismo $F_{2}: \mathbb{C}^{\phi_{2}\left(L_{1}\right)} \longrightarrow Y_{1}$.

Prosseguindo de maneira análoga para cada $z_{i}, 1 \leq i \leq n-1$, construímos $(n-1)$ polinômios 2-homogêneos e encontramos um subespaço $Y_{n-1} \subset Z_{1}$ tal que $\operatorname{dim} Y_{n-1}=\phi_{2}\left(\stackrel{(n-1)}{\cdots}\left(\phi_{2}\left(L_{1}\right) \ldots\right)\right)$ e $A\left(z_{1}, x, x\right)=\ldots=A\left(z_{n-1}, x, x\right)=0$ para cada $x \in Y_{n-1}$. Observamos que $(n-1)$ nada mais é que o número de soluções inteiras e não negativas da equação $s_{1}+\ldots+s_{n-1}=1$. Vamos denotar por $L_{2}=\phi_{2}\left(\stackrel{(n-1)}{\cdots}\left(\phi_{2}\left(L_{1}\right) \ldots\right)\right)$. Suponhamos que o valor de $m$ que estamos determinando seja tal que $L_{2} \geq 2$. Logo 
existe $G: \mathbb{C}^{L_{2}} \longrightarrow Y_{n-1}$ um isomorfismo. Também temos que $\left.P\right|_{Y_{n-1}}$ é um polinômio 3-homogêneo. Assim $\left.P\right|_{Y_{n-1}} \circ G: \mathbb{C}^{L_{2}} \longrightarrow Y_{n-1} \longrightarrow \mathbb{C}$ é um polinômio 3-homogêneo.

Como $L_{2} \geq 2$, por $(\mathcal{P} 1)$ existe $x_{n} \in \mathbb{C}^{L_{2}}$ não-nulo tal que $\left.P\right|_{Y_{n-1}} \circ G\left(x_{n}\right)=0$. Tomemos $z_{n}=G\left(x_{n}\right)$. Então $P\left(z_{n}\right)=0$ e $\left\{z_{1}, \ldots, z_{n}\right\}$ é linearmente independente, pois $z_{n} \in Y$. Por $(\mathcal{P} 2)$ temos que $\left[z_{1}, \ldots, z_{n}\right] \subset P^{-1}(0)$. Por definição de $L_{2}$, temos que $L_{2}=\left\lceil\frac{\left\lceil\frac{\left\lceil\frac{L_{1}}{2}\right\rceil}{2}\right\rceil}{2}\right\rceil$ $(n-1)$ vezes. Do lema 4.2.1 segue que $k_{1}=\frac{n^{2}-n}{2}$ e assim $L_{1}=m-n+1-\left(\frac{n^{2}-n}{2}\right)$. Logo, como $L_{2} \geq 2$ temos que $m \geq 2^{n}+\left(\frac{n^{2}+n}{2}\right)-1$.

Portanto, para cada $n \in \mathbb{N}$, temos que $\theta_{3}(n) \leq 2^{n}+\left(\frac{n^{2}+n}{2}\right)-1$.

Segue diretamente da demonstração que para cada $m \in \mathbb{N}$,

$$
\phi_{3}(m) \geq \max \left\{n \in \mathbb{N}_{0}: m \geq 2^{n}+\left(\frac{n^{2}+n}{2}\right)-1\right\} .
$$

Observamos que, por esta demonstração, $\theta_{3}(3) \leq 13, \theta_{3}(4) \leq 25$ e $\theta_{3}(5) \leq 46$. E pela fórmula obtida por Aron e Rueda, $\theta_{3}(3) \leq 16, \theta_{3}(4) \leq 40$ e $\theta_{3}(5) \leq 96$.

Na próxima proposição vamos calcular uma limitação para $\theta_{4}(n)$. Aqui também para $n=1,2$ o valor da limitação aqui encontrada coincide com o valor apresentado por Aron e Rueda. Para $n \geq 3$, a limitação aqui encontrada assume valores bem menores que os apresentados em [9].

Para cada $j \in \mathbb{N}_{0}$, definimos: $K_{j}=\left\{\begin{array}{cc}j=0 \\ 2^{K_{j-1}+\frac{K_{j-1}^{2}+K_{j-1}}{2}-1 ;} \quad j \geq 1\end{array}\right.$.

Proposição 4.2.4. Para $d=4$, para cada $n \in \mathbb{N}$, temos que

$$
\theta_{4}(n) \leq 2^{\frac{n^{2}-n}{2}} K_{n}+n+\frac{n^{3}-n}{6}-1
$$

Demonstração: A demonstração será feita por indução sobre $n$. Para $n=1$, pela observação $4.2 .2, \theta_{4}(1)=2$. 
Suponhamos que por indução já calculamos as limitações para $\theta_{4}(1), \ldots, \theta_{4}(n-1)$ e digamos que $\theta_{4}(n-1) \leq M$ para algum $M \in \mathbb{N}$. Sejam $m \geq M+1$ (a ser determinado), $P: \mathbb{C}^{m} \longrightarrow \mathbb{C}$ um polinômio 4-homogêneo não nulo e $A: \mathbb{C}^{m} \times \mathbb{C}^{m} \times \mathbb{C}^{m} \longrightarrow \mathbb{C}$ a aplicação 4-linear simétrica associada a $P$. Como $m \geq M+1$ então existem $z_{1}, \ldots, z_{n-1} \in \mathbb{C}^{m}$ tais que $\left\{z_{1}, \ldots, z_{n-1}\right\}$ é linearmente independente e $\left[z_{1}, \ldots, z_{n-1}\right] \subset P^{-1}(0)$.

Podemos escrever $\mathbb{C}^{m}=\left[z_{1}, \ldots, z_{n-1}\right] \oplus Y$, onde $\operatorname{dim} Y=m-n+1$, e existe $T_{1}: \mathbb{C}^{m-n+1} \longrightarrow Y$ um isomorfismo. Com raciocínio análogo ao da proposição 4.2 .3 para cada terna $\left(z_{i}, z_{j}, z_{l}\right)$ com $1 \leq i, j, l \leq n-1$, construímos $k_{1}$ funcionais lineares e encontramos um subespaço $Y_{1} \subset Y$ tal que $A\left(z_{i}^{s_{i}}, z_{j}^{s_{j}}, z_{l}^{s_{l}}, x\right)=0$ para cada $x \in Y_{1}$ e cada $(n-1)$ upla $\left(s_{1}, \ldots, s_{n-1}\right) \in\left(\mathbb{N}_{0}\right)^{n-1} \operatorname{com} s_{1}+\ldots+s_{n-1}=3$ e $\operatorname{dim} Y_{1}=\phi_{1}\left(\stackrel{\left(k_{1}\right)}{*}\left(\phi_{1}(m-n+1) \ldots\right)\right)$, onde $k_{1}$ é o número de soluções inteiras e não negativas da equação $s_{1}+\ldots+s_{n-1}=3$. Seja $L_{1}=\phi_{1}\left(\stackrel{\left(k_{1}\right)}{*}\left(\phi_{1}(m-n+1) \ldots\right)\right)$ e suponhamos que o valor de $m$ que estamos determinando seja tal que $L_{1} \geq 2$. Assim existe um isomorfismo $T: \mathbb{C}^{L_{1}} \longrightarrow Y_{1}$.

Prosseguindo para cada par $\left(z_{i}, z_{j}\right)$ com $1 \leq i \leq j \leq n-1$, agora construímos $k_{2}$ polinômios 2-homogêneos e encontramos um subespaço $Y_{2} \subset Y_{1}$ tal que $A\left(z_{i}^{s_{i}}, z_{j}^{s_{j}}, x, x\right)=0$ para cada $x \in Y_{2}$ e cada $(n-1)$-upla $\left(s_{1}, \ldots, s_{n-1}\right) \in\left(\mathbb{N}_{0}\right)^{n-1} \operatorname{com} s_{1}+\ldots+s_{n-1}=2 \mathrm{e}$ $\operatorname{dim} Y_{2}=\phi_{2}\left(\stackrel{\left(k_{2}\right)}{*}\left(\phi_{2}\left(L_{1}\right) \ldots\right)\right)$, onde $k_{2}$ é o número de soluções inteiras e não negativas da equação $s_{1}+\ldots+s_{n-1}=2$.

Seja $L_{2}=\phi_{2}\left(\stackrel{\left(k_{2}\right)}{*}\left(\phi_{2}\left(L_{1}\right) \ldots\right)\right)$ e suponhamos que o valor de $m$ que estamos determinando seja tal que $L_{2} \geq 2$, logo existe um isomorfismo $T_{3}: \mathbb{C}^{L_{2}} \longrightarrow Y_{2}$.

Prosseguindo para cada $z_{i}, 1 \leq i \leq n-1$, construímos $(n-1)$ polinômios 3-homogêneos e encontramos um subespaço $Y_{3} \subset Y_{2}$ tal que $A\left(z_{1}, x, x, x\right)=\ldots=A\left(z_{n-1}, x, x, x\right)=0$ para cada $x \in Y_{3}$ e $\operatorname{dim} Y_{3}=\phi_{3}\left(\stackrel{(n-1)}{\cdots}\left(\phi_{3}\left(L_{2}\right) \ldots\right)\right)$. Observamos que $(n-1)$ é o número de soluções inteiras e não negativas da equação $s_{1}+\ldots+s_{n-1}=1$.

Seja $L_{3}=\phi_{3}\left(\stackrel{(n-1)}{\cdots}\left(\phi_{3}\left(L_{2}\right) \ldots\right)\right)$ tal que $L_{3} \geq 2$. Então existe um isomorfismo $H: \mathbb{C}^{L_{3}} \longrightarrow Y_{3}$. Também temos que $\left.P\right|_{Y_{3}}$ é um polinômio 4-homogêneo. Assim $\left.P\right|_{Y_{3}} \circ H: \mathbb{C}^{L_{3}} \longrightarrow Y_{3} \longrightarrow \mathbb{C}$ é um polinômio 4-homogêneo.

Como $L_{3} \geq 2$, por $(\mathcal{P} 1)$ existe $x_{n} \in \mathbb{C}^{L_{3}}$ não nulo tal que $\left.P\right|_{Y_{3}} \circ H\left(x_{n}\right)=0$. Tomemos $z_{n}=H\left(x_{n}\right)$. Então $P\left(z_{n}\right)=0$ e $\left\{z_{1}, \ldots, z_{n}\right\}$ é linearmente independente, pois $z_{n} \in Y$. Por $(\mathcal{P} 2)$ temos que $\left[z_{1}, \ldots, z_{n}\right] \subset P^{-1}(0)$. 
Seja $K_{1}:=2$, sendo $L_{3} \geq 2=K_{1}$ segue da proposição 4.2 .3 que $K_{2}:=6 \leq \phi_{3}\left({ }^{(n-2)}\left(\phi_{3}\left(L_{2}\right) \ldots\right)\right)$ e $\operatorname{assim} K_{3}:=2^{6}+\frac{6^{2}+6}{2}-1 \leq \phi_{3}\left({ }^{(n-3)}\left(\phi_{3}\left(L_{2}\right) \ldots\right)\right)$. Prosseguindo, obtemos $L_{2} \geq 2^{K_{n-1}}+\frac{K_{n-1}^{2}+K_{n-1}}{2}-1:=K_{n}$. Da proposição 4.1 .2 temos que $L_{2}=\left\lceil\frac{\left\lceil\frac{\left\lceil\frac{L_{1}}{2}\right\rceil}{2}\right\rceil}{2}\right\rceil$ $\left(k_{2}\right)$ vezes. Como $L_{1}=m-n+1-k_{1}$ segue que $K_{n} \leq \frac{m-n-k_{1}+1}{2^{k_{2}}}$. Desta maneira, $m \geq 2^{k_{2}} K_{n}+n+k_{1}-1$. Do lema 4.2.1 temos que $k_{1}=\frac{n^{3}-n}{6}$ e $k_{2}=\frac{n^{2}-n}{2}$. Portanto, para cada $n \in \mathbb{N}$, temos que $\theta_{4}(n) \leq 2^{\frac{n^{2}-n}{2}} K_{n}+n+\frac{n^{3}-n}{6}-1$.

Segue diretamente da demonstração que para cada $m \in \mathbb{N}$,

$$
\phi_{4}(m) \geq \max \left\{n \in \mathbb{N}_{0}: m \geq 2^{\frac{n^{2}-n}{2}} K_{n}+n+\frac{n^{3}-n}{6}-1\right\} .
$$

Observamos que aqui, $\theta_{4}(3) \leq 678$ e $\theta_{4}(4) \leq 2^{90}+2^{6} \cdot 11729+13$. Enquanto que por Aron e Rueda $\theta_{4}(3) \leq 2^{28} \cdot 29+2$ e $\theta_{4}(4) \leq 2^{2^{39} \cdot 38}\left[2^{39} \cdot 38+2^{2}+1\right]+2$.

Já vimos anteriormente, $\theta_{1}(n)=n+1:=M_{1}(n), \theta_{2}(n)=2 n:=M_{2}(n)$, $\theta_{3}(n) \leq 2^{n}+\frac{n^{2}+n}{2}-1:=M_{3}(n), \theta_{4}(n) \leq 2^{\frac{n^{2}-n}{2}} K_{n}+n+\frac{n^{3}-n}{6}-1:=M_{4}(n)$. Agora vamos supor que conhecemos limitações de $\theta_{5}(n), \theta_{6}(n), \ldots, \theta_{d-1}(n)$ para cada $n \in \mathbb{N}$ com $d \geq 5$. Digamos que $\theta_{5}(n) \leq M_{5}(n), \ldots, \theta_{d-1}(n) \leq M_{d-1}(n)$ para cada $n \in \mathbb{N}$, onde $M_{j}, 1 \leq j \leq d-1$, são as funções limitações que dependem de $n$ e $j$. Assim, $\phi_{1}(m)=m-1, \phi_{2}(m)=\left\lceil\frac{m}{2}\right\rceil, \phi_{3}(m) \geq \max \left\{n \in \mathbb{N}_{0}: m \geq M_{3}(n)\right\}, \ldots$, $\phi_{d-1}(m) \geq \max \left\{n \in \mathbb{N}_{0}: m \geq M_{d-1}(n)\right\}$.

Agora vamos calcular a limitação para $\theta_{d}(n)$.

Dado $n \in \mathbb{N}$ com $n \geq 2$, para cada $1 \leq j \leq d$ seja $k_{j}$ o número de soluções inteiras e não negativas da equação $s_{1}+\ldots+s_{n-1}=d-j$. Do lema 4.2 .1 segue que $k_{j}=\frac{(n+d-j-2) !}{(d-j) !(n-2) !}$. Para cada $0 \leq l \leq d-3$, definimos: $N_{l}=\left\{\begin{array}{cc}l=0 \\ 2 ; \\ M_{d-l}\left(\stackrel{\left(k_{d-l}\right)}{ }\right)\left(M_{d-l}\left(N_{l-1}\right) \ldots\right) ; & l \geq 1\end{array}\right.$.

Teorema 4.2.5. Seja $d \in \mathbb{N}$ com $d \geq 5$ e suponhamos que as limitações para $\theta_{1}(n), \theta_{2}(n), \ldots, \theta_{d-1}(n)$ são determinadas para cada $n \in \mathbb{N}$. Então, $\theta_{d}(1)=2$ e para cada 
$n \geq 2, \theta_{d}(n) \leq 2^{\frac{(n+d-4) !}{(d-2) !(n-2) !}} N_{d-3}+n+\frac{(n+d-3) !}{(d-1) !(n-2) !}-1$.

Demonstração: Para calcular a limitação de $\theta_{d}(n)$, a demonstração será feita por indução sobre $n$. Para $n=1$, pela observação $4.2 .2, \theta_{d}(1)=2$.

Suponhamos que por indução já calculamos as limitações para $\theta_{d}(1), \ldots, \theta_{d}(n-1)$ e digamos que $\theta_{d}(n-1) \leq M$ para algum $M \in \mathbb{N}$. Vamos determinar a limitação para $\theta_{d}(n)$, ou seja, o menor $m$ tal que para cada polinômio $d$-homogêneo $P: \mathbb{C}^{m} \longrightarrow \mathbb{C}$, o conjunto $P^{-1}(0)$ contenha um subespaço $n$-dimensional. Sejam $m \geq M+1$ (a ser determinado), $P: \mathbb{C}^{m} \longrightarrow \mathbb{C}$ um polinômio d-homogêneo não nulo e $\left.A: \mathbb{C}^{m} \times \stackrel{(d)}{ }\right) \times \mathbb{C}^{m} \longrightarrow \mathbb{C}$ a aplicação $d$-linear simétrica associada a $P$. Como $m \geq M+1$ então existem $z_{1}, \ldots, z_{n-1} \in \mathbb{C}^{m}$ tais que $\left\{z_{1}, \ldots, z_{n-1}\right\}$ é linearmente independente e $\left[z_{1}, \ldots, z_{n-1}\right] \subset P^{-1}(0)$.

Podemos escrever $\mathbb{C}^{m}=\left[z_{1}, \ldots, z_{n-1}\right] \oplus Y$, onde $\operatorname{dim} Y=m-n+1$ e existe $T_{1}: \mathbb{C}^{m-n+1} \longrightarrow Y$ um isomorfismo. Com raciocínio análogo ao da proposição 4.2 .3 para cada $(d-1)$-uplas $\left(z_{i_{1}}, \ldots, z_{i_{d-1}}\right)$ com $1 \leq i_{t} \leq n-1$ para cada $1 \leq t \leq d-1$, construímos $k_{1}$ funcionais lineares e encontramos um subespaço $X_{1} \subset Y$ tal que $A\left(z_{1}^{s_{1}}, \ldots, z_{n-1}^{s_{n-1}}, x\right)=0$ para cada $x \in X_{1}$ e cada $(n-1)$-upla $\left(s_{1}, \ldots, s_{n-1}\right) \in\left(\mathbb{N}_{0}\right)^{n-1}$ com $s_{1}+\ldots+s_{n-1}=d-1$ e $\left.\operatorname{dim} X_{1}=\phi_{1}(\stackrel{(k 1}{1})^{\prime}\left(\phi_{1}(m-n+1) \ldots\right)\right)$, onde $k_{1}$ é o número de soluções inteiras e não negativas da equação $s_{1}+\ldots+s_{n-1}=d-1$.

Seja $L_{1}=\phi_{1}\left({ }^{\left(k_{1}\right)}\left(\phi_{1}(m-n+1) \ldots\right)\right)$ tal que $L_{1} \geq 2$. Assim existe um isomorfismo $T_{2}: \mathbb{C}^{L_{1}} \longrightarrow X_{1}$.

Agora para as $(d-2)$-uplas $\left(z_{i_{1}}, \ldots, z_{i_{d-2}}\right)$ com $1 \leq i_{t} \leq n-1$ para cada $1 \leq t \leq d-2$, construímos $k_{2}$ polinômios 2-homogêneos e encontramos um subespaço $X_{2} \subset X_{1}$ tal que $A\left(z_{1}^{s_{1}}, \ldots, z_{n-1}^{s_{n-1}}, x, x\right)=0$ para cada $x \in X_{2}$ e cada $(n-1)$-upla $\left(s_{1}, \ldots, s_{n-1}\right) \in\left(\mathbb{N}_{0}\right)^{n-1}$ com $s_{1}+\ldots+s_{n-1}=d-2$ e $\operatorname{dim} X_{2}=\phi_{2}\left(\stackrel{\left(k_{2}\right)}{*}\left(\phi_{2}\left(L_{1}\right) \ldots\right)\right)$, onde $k_{2}$ é o número de soluções inteiras e não negativas da equação $s_{1}+\ldots+s_{n-1}=d-2$.

Prosseguindo de maneira análoga, construímos $k_{j}$ polinômios $j$-homogêneos para cada $j$, $3 \leq j \leq d-1$, e encontramos um subespaço $X \subset X_{2}$ tal que $A\left(z_{1}^{s_{1}}, \ldots, z_{n-1}^{s_{n-1}}, x^{j}\right)=0$ para cada $x \in X$, cada $j, 3 \leq j \leq d-1$, cada $(n-1)$-upla $\left(s_{1}, \ldots, s_{n-1}\right) \in\left(\mathbb{N}_{0}\right)^{n-1}$ com $s_{1}+\ldots+s_{n-1}=d-j$ e $\operatorname{dim} X=\phi_{d-1}\left(\stackrel{(n-1)}{*}\left(\phi_{d-1}\left(L_{d-2}\right) \ldots\right)\right)$. Observamos que $(n-1)$ é o número de soluções inteiras e não negativas da equação $s_{1}+\ldots+s_{n-1}=1$ e aqui é o 
nosso $k_{d-1}$.

Seja $L_{d-1}=\phi_{d-1}\left(\stackrel{(n-1)}{\cdots}\left(\phi_{d-1}\left(L_{d-2}\right) \ldots\right)\right)$ e suponhamos que o valor de $m$ que estamos determinando seja tal que $L_{d-1} \geq 2$. Então existe $H: \mathbb{C}^{L_{d-1}} \longrightarrow X$ um isomorfismo. Também temos que $\left.P\right|_{X}$ é um polinômio $d$-homogêneo. Assim $\left.P\right|_{X} \circ H: \mathbb{C}^{L_{d-1}} \longrightarrow X \longrightarrow \mathbb{C}$ é um polinômio $d$-homogêneo.

Como $L_{d-1} \geq 2$, por $(\mathcal{P} 1)$ existe $x_{n}$ não nulo em $\mathbb{C}^{L_{d-1}}$ tal que $\left.P\right|_{X} \circ H\left(x_{n}\right)=0$. Vamos tomar $z_{n}=H\left(x_{n}\right)$. Então $\left\{z_{1}, \ldots, z_{n}\right\}$ é linearmente independente, pois $z_{n} \in Y$ e $P\left(z_{n}\right)=0$. De $(\mathcal{P} 2)$ segue que $\left[z_{1}, \ldots, z_{n}\right] \subset P^{-1}(0)$.

Seja $N_{0}=2$. Sendo $L_{d-1} \geq 2:=N_{0}$ segue que $N_{1}:=M_{d-1}\left(\stackrel{(n-1)}{\cdots} M_{d-1}\left(N_{0}\right) \ldots\right) \leq L_{d-2}$ e $\operatorname{assim} L_{d-3} \geq M_{d-2}\left({ }^{\left(k_{d-2}\right)} M_{d-2}\left(N_{1}\right) \ldots\right):=N_{2}$. Prosseguindo, temos que $N_{d-3} \leq L_{2}$. Da proposição 4.1 .2 temos que $L_{2} \leq \frac{L_{1}}{2^{k_{2}}}$. Como $L_{1}=m-n+1-k_{1}$ segue que $m \geq 2^{k_{2}} N_{d-3}+n+k_{1}-1$. Do lema 4.2.1, temos que $k_{1}=\frac{(n+d-3) !}{(d-1) !(n-2) !}$ e $k_{2}=\frac{(n+d-4) !}{(d-2) !(n-2) !}$. Portanto, para cada $n \in \mathbb{N}$, temos que $\theta_{d}(n) \leq 2^{\frac{(n+d-4) !}{(d-2) !(n-2) !}} N_{d-3}+n+\frac{(n+d-3) !}{(d-1) !(n-2) !}-1$.

Segue imediatamente da demonstração que:

$\phi_{d}(1)=0$ e $\phi_{d}(m) \geq 1$ se $2 \leq m<2 N_{d-3}+2$, e para $m \geq 2 N_{d-3}+2$, $\phi_{d}(m) \geq \max \left\{n \in \mathbb{N}: m \geq 2^{\frac{(n+d-4) !}{(d-2) !(n-2) !}} N_{d-3}+n+\frac{(n+d-3) !}{(d-1) !(n-2) !}-1\right\}$.

A proposição 4.1 .2 nos dá a melhor limitação $\theta_{2}(n)$ para cada $n \in \mathbb{N}$. Utilizando-se da mesma técnica de demonstração temos:

Teorema 4.2.6. Existe uma função $g: \mathbb{N} \times \mathbb{N} \longrightarrow \mathbb{N}_{0}$ tais que para $m, n \in \mathbb{N}$ com $m \geq \theta_{2}(n)$ e $P \in \mathcal{P}\left({ }^{2} \mathbb{C}^{m}\right)$ existem $x_{1}, \ldots, x_{n} \in \mathbb{C}^{m}$ linearmente independentes e um subespaço $X \subset \mathbb{C}^{m}$ com $\operatorname{dim} X=g(m, n)$ tais que para quaisquer 2 vetores $v_{1}, v_{2} \in\left[x_{1}, \ldots, x_{n}\right] \oplus X$ temos que $A\left(v_{1}, v_{2}\right)=0$ sempre que no mínimo algum $v_{i} \in\left[x_{1}, \ldots, x_{n}\right]$ para algum $i=1,2$. Além disso, se $g(m, n) \geq 2$ então existem $x_{n+1} \in X$ e um subespaço $X_{1} \subset \mathbb{C}^{m}$ tais que $P\left(x_{n+1}\right)=0, \operatorname{dim} X_{1}=g(m, n+1)$ e para quaisquer 2 vetores $v_{1}, v_{2} \in\left[x_{1}, \ldots, x_{n+1}\right] \oplus X_{1}$ temos que $A\left(v_{1}, v_{2}\right)=0$ sempre que no mínimo algum $v_{i}=\left[x_{1}, \ldots, x_{n+1}\right]$ para algum $i=1,2$. 
Demonstração: Definamos $g: \mathbb{N} \times \mathbb{N} \longrightarrow \mathbb{N}_{0}$ da seguinte maneira:

$$
g(m, n)= \begin{cases}m-2 n ; & m \geq 2 n \\ 0 ; & m<2 n\end{cases}
$$

Sejam $m \geq \theta_{2}(n), P: \mathbb{C}^{m} \longrightarrow \mathbb{C}$ um polinômio 2-homogêneo e $A$ a aplicação 2-linear simétrica associada a $P$. Pela proposição 4.1 .2 temos que $\theta_{2}(n)=2 n$, $\log$ o $m \geq 2 n$. Assim existem $x_{1}, \ldots, x_{n} \in \mathbb{C}^{m}$ linearmente independentes tais que $\left[x_{1}, \ldots, x_{n}\right] \subset P^{-1}(0)$. Podemos escrever $\mathbb{C}^{m}=\left[x_{1}, \ldots, x_{n}\right] \oplus Y \operatorname{com} \operatorname{dim} Y=m-n$, pois $m>n$. Se $m=2 n$ basta tomar $X=\{0\}$. Se $m>2 n$, de maneira análoga à demonstração da proposição 4.2.3, existe um subespaço $X \subset Y$ tal que $A\left(x_{i}^{s_{i}}, x\right)=0$ para cada $x \in X$ e cada $n$-upla $\left(s_{1}, \ldots, s_{n}\right) \in\left(\mathbb{N}_{0}\right)^{n}$ com $s_{1}+\ldots+s_{n}=1$ e $\operatorname{dim} X=\phi_{1}\left(\stackrel{(n)}{.}\left(\phi_{1}(m-n), \ldots\right)\right)$. Pela bilinearidade, para quaisquer 2-vetores $v_{1}, v_{2} \in\left[x_{1}, \ldots, x_{n}\right] \oplus X$ temos que $A\left(v_{1}, v_{2}\right)=0$ sempre que no mínimo algum $v_{i} \in\left[x_{1}, \ldots, x_{n}\right]$ para algum $i=1,2$. Como $m>2 n$ e $\operatorname{dim} X=m-n-n=m-2 n$ temos que $\operatorname{dim} X=g(m, n)$. Então existe $T: \mathbb{C}^{g(m, n)} \longrightarrow X$ um isomorfismo. Como $\left.P\right|_{X}$ é 2-homogêneo temos que $Q=\left.P\right|_{X} \circ T$ é 2-homogêneo.

Se $m$ é tal que $g(m, n) \geq 2$, ou seja, $m \geq 2(n+1)$, por $(\mathcal{P} 1)$ existe $x_{n+1}$ não nulo em $X$ tal que $P\left(x_{n+1}\right)=0,\left\{x_{1}, \ldots, x_{n+1}\right\}$ é linearmente independente e por $(\mathcal{P} 2)$ $\left[x_{1}, \ldots, x_{n+1}\right] \subset P^{-1}(0)$. Então podemos escrever $\mathbb{C}^{m}=\left[x_{1}, \ldots, x_{n+1}\right] \oplus Y_{1}$ com $\operatorname{dim} Y_{1}=m-(n+1)$, pois $m>2 n$. Se $m=2(n+1)$, basta tomar $X_{1}=\{0\}$. Se $m>2(n+1)$, de maneira análoga à demonstração da proposição 4.2.3, existe um subespaço $X_{1} \subset Y_{1}$ tal que $A\left(x_{i}^{s_{i}}, x\right)=0$ para cada $x \in X_{1}$ e cada $(n+1)$-upla $\left(s_{1}, \ldots, s_{n+1}\right) \in\left(\mathbb{N}_{0}\right)^{n+1} \operatorname{com} s_{1}+\ldots+s_{n+1}=1 \mathrm{e}$ $\operatorname{dim} X_{1}=\phi_{1}\left({ }^{(n+1)}\left(\phi_{1}(m-(n+1)), \ldots\right)\right)$. Ou seja, para quaisquer 2-vetores $v_{1}, v_{2} \in\left[x_{1}, \ldots, x_{n+1}\right] \oplus X_{1}$ temos que $A\left(v_{1}, v_{2}\right)=0$ sempre que no mínimo algum $v_{i} \in\left[x_{1}, \ldots, x_{n+1}\right]$ para algum $i=1,2$ (pela bilinearidade). $\mathrm{E} \operatorname{dim} X_{1}=m-(n+1)-(n+1)=m-2(n+1)=g(m, n+1)$, pois $m>2(n+1)$.

Para finalizar, observamos que se conhecemos a melhor melhor função $\theta: \mathbb{N} \times \mathbb{N} \longrightarrow \mathbb{N}$, e conseqüentemente temos a melhor função $\phi: \mathbb{N} \times \mathbb{N} \longrightarrow \mathbb{N}_{0}$ podemos definir uma função $f: \mathbb{N} \times \mathbb{N} \times \mathbb{N} \longrightarrow \mathbb{N}_{0}$ com as seguintes propriedades: 
Para cada $m, d \in \mathbb{N}$ com $m \geq \theta(n, d)$ e para cada $P \in \mathcal{P}\left({ }^{d} \mathbb{C}^{m}\right)$ existem um conjunto linearmente independente $\left\{x_{1}, \ldots, x_{n}\right\} \in \mathbb{C}^{m}$ e um subespaço $X \subset \mathbb{C}^{m}$ com $\operatorname{dim} X=f(m, n, d)$ tal que para cada $d$ vetores $v_{1}, \ldots, v_{d} \in\left[x_{1}, \ldots, x_{n}\right] \oplus X$ temos que $A\left(v_{1}, \ldots, v_{d}\right)=0$ sempre que no mínimo algum $v_{i} \in\left[x_{1}, \ldots, x_{n}\right]$ para algum $i, 1 \leq i \leq d$. Além disso, se $f(m, n, d) \geq 2$ existem $x_{n+1} \in X$ e um subespaço $X_{1} \subset \mathbb{C}^{m}$ tais que $P\left(x_{n+1}\right)=0, \operatorname{dim} X_{1}=f(m, n+1, d)$ e para quaisquer vetores $v_{1}, \ldots, v_{d} \in\left[x_{1}, \ldots, x_{n+1}\right] \oplus$ $X_{1}$ temos que $A\left(v_{1}, \ldots, v_{d}\right)=0$ sempre que no mínimo algum $v_{i} \in\left[x_{1}, \ldots, x_{n+1}\right]$ para algum $i, 1 \leq i \leq d$.

Vamos assumir que conhecemos a melhor função $\phi: \mathbb{N} \times \mathbb{N} \longrightarrow \mathbb{N}_{0}$. Então podemos definir a função $f: \mathbb{N} \times \mathbb{N} \times \mathbb{N} \longrightarrow \mathbb{N}_{0}$ da seguinte maneira:

Para $d=1$,

$$
f(m, n, 1)= \begin{cases}m-n ; & m \geq n \\ 0 ; & m<n\end{cases}
$$

Para $d=2$,

$$
f(m, n, 2)= \begin{cases}m-2 n ; & m \geq 2 n \\ 0 ; & m<2 n\end{cases}
$$

Para $d \geq 3$ e $n=1$,

$$
f(m, 1, d)=\left\{\begin{array}{ll}
W_{d-1} ; & W_{d-2} \geq 1 \\
0 ; & m<2 \text { ou } W_{j}=0 \text { para algum } 1 \leq j \leq d-2
\end{array},\right.
$$

onde $W_{s}=\phi(\ldots(\phi(m-1,1), 2), \ldots, s)$ para cada $s, 1 \leq s \leq d-1$.

Para $d \geq 3$ e para cada $n \geq 2$, seja $w_{l}=\frac{(n+d-l-1) !}{(d-l) !(n-1) !}$, dado pelo lema $4.2 .1(1 \leq l \leq d-2)$. Agora, consideremos $S_{1}=m-n-w_{1}$ e $\left.S_{j}=\phi(\stackrel{(w}{j})^{\prime}\left(\phi\left(S_{j-1}, j\right), \ldots\right), j\right)$ para cada $j$, $2 \leq j \leq d-1$. Nós definimos

$$
f(m, n, d)=\left\{\begin{array}{ll}
S_{d-1} ; & \phi\left(\stackrel{(n-1)}{\cdots}\left(\phi\left(S_{d-2}, d-1\right), \ldots\right), d-1\right) \geq 1 \\
0 ; & m<n+w_{1} \text { ou } S_{j}=0 \text { para algum } 1 \leq j \leq d-2 \text { ou } \\
& \phi(s, t)=0 \text { para algum } 2 \leq t \leq d-1 \\
& \text { e algum } \phi\left(S_{j-1}, j\right) \leq s \leq \phi\left(\stackrel{\left(w_{j}-1\right)}{\cdots}\left(\phi\left(S_{j-1}, j\right), \ldots\right), j\right)
\end{array} .\right.
$$




\section{Referências Bibliográficas}

[1] R. Alencar, R.M. Aron and S. Dineen, A reflexive of holomorphic functions in infnitely many variables, Proc. Amer. Math. Soc. 90 (1984), $n^{\underline{o}}$ 3, 407-411.

[2] R. Alencar and K. Floret, Weak-strong continuity of multilinear mapping and the Pelczyński-Pitt theorem, J. Math. A. Appl. 206 (1997), no 2, 532-546.

[3] R.M. Aron, C. Boyd and Y.S. Choi, Unique Hahn-Banach theorems for spaces of homogeneos polynomials, J. Austral. Math. Soc. 70 (2001), $n^{-0}$ 3, 387-400.

[4] R.M. Aron, Y.S. Choi and J.G. Llavona, Estimates by polynomials, Bull. Austral. Math. Soc. 52 (1995), nº 3, 475-486.

[5] R.M. Aron and V. Dimant, Sets of weak sequential continuity for polynomials, Indag. Math. 13 (2002), $n^{\underline{0}}$ 3, 287-299.

[6] R.M. Aron and S. Dineen, Q-reflexive Banach space, Rocky Mountain J. Math. 27 (1997), $n^{\circ}$ 4, 1009-1025.

[7] R.M. Aron, C. Hervés and M. Valdivia, Weakly continuous mappings on Banach spaces, J. Funct. Anal. 52 (1983), no 2, 189-204.

[8] R.M. Aron and J.B. Prolla, Polynomial approximation of differentiable functions on Banach spaces, J. Reine Angew. Math. 313 (1980), 195-216.

[9] R.M. Aron and M.P. Rueda, A problem concerning zero-subspaces of homogeneous polynomials, Linear Top. Spaces and Complex Analysis 3 (1997), 20-23. 
[10] A.A. Baernstein II, On reflexivity and summability, Studia Math. 42 (1972), $n^{\underline{o}}$ 1, 91-94.

[11] C. Boyd and R. Ryan,Bounded weak continuity of homogeneous polynomials at the origin, Arch. Math. 71 (1998) 211-218.

[12] J.R. Calder and J.B. Hill, A collection of sequence space, Trans. Amer. Math. Soc. 152 (1970), $n^{\underline{o}} 1,107-118$.

[13] D. Carando and V. Dimant, Duality in spaces of nuclear and integral polynomials, J. Math. Analysis and Appl. 241 (2000), $n^{\underline{o}}$ 1, 107-121.

[14] H. Carrión, P. Galindo and M.L. Lourenço, Banach spaces whose bounded sets are bounding in the bidual, Ann. Acad. Sci. Fenn. Math. (2006), a aparecer.

[15] J.M.F. Castillo, R. García and R. Gonzalo, Banach spaces in which all multilinear forms are weakly sequentially continuous, Studia Math. 136 (1999), no 2, 121-145.

[16] J.M.F. Castillo and F. Sánchez, Remarks on some basic properties of Tsirelson's space, Note di Mat. 13 (1993), $n^{\underline{o}}$ 1, 117-122.

[17] _ Weakly p-compact, p-Banach-Saks and super-reflexive Banach spaces, J. Math. Analysis and Appl. 185 (1994), no 2, 256-261.

[18] P.G. Casazza and T.J. Shura, Tsirelson's space, Lecture Notes in Mathematics, vol. 1363, Springer-Verlag, Berlin, 1989.

[19] Y.S. Choi and S.G. Kim, Polynomial properties of Banach spaces, J. Math. Anal. Appl. 190 (1995), $n^{\underline{o}}$ 1, 203-210.

[20] D.J.H. Garling, On symmetric sequences spaces, Proc. London Math. Soc. 16 (1966), $n^{\underline{o}} 1-2,85-106$.

[21] _ _ A class of reflexive symmetric BK-space, Canadian J. Math. 21 (1969), $n^{\text {o }}$ 1, 602-608. 
[22] M. González and J.M. Gutiérrez, Polynomials on Schreier's space, Rocky Mountain J. Math. 30 (2000), $n^{\underline{o}}$ 2, 571-585.

[23] R. Gonzalo, Upper and lower estimates in Banach spaces, Comment. Math. Univ. Carolinae 36 (1995), $n^{\underline{o}}$ 4, 641-653.

[24] A. Grothendieck, Sur certaines classes de suites dan les espaces de Banach et le theoréme de Dvoretzsky Rogers, Boletin Sociedad Mathematica Sao Paulo, 8 (1956), 85-110.

[25] A.J. Hissadomi, Propriedade de Dunford-Pettis Polinomial e Espaços Polinomialmente de Schur, Dissertação de Mestrado, IME-USP 1998.

[26] J. Lindenstrauss and L. Tzafiri, Classical Banach Spaces I and II. Classics in Mathematics, Springer-Verlag, London, 1996.

[27] J.G. Llavona and L.A. Moraes, The Aron Berner extension for polynomials defined in the dual of a Banach space, Publ. Res. Inst. Math. Sci. 40 (2004), $n^{\underline{o}}$ 1, 221-230.

[28] M.L. Lourenço and L.A. Moraes, A class of polynomials from Banach spaces into Banach algebras, Publ. Res. Inst. Math. Sci. 37 (2001), no 4, 521-529.

[29] M.L. Lourenço and N.N. Tocha, Zeros of complex homogeneous polynomials, Linear and Multilinear Algebra (2006), a aparecer.

[30] R.E. Megginson, An introduction to Banach spaces theory. Graduate Texts in Mathematics, Springer-Verlag, New York, 1998.

[31] J. Mujica, Complex Analysis in Banach Spaces, North-Holland Math. Studies, vol. 120, North-Holland, Amsterdam, 1986.

[32] _ _ Banach space not containing $l_{1}$, Ark. Mat. 41 (2003), $n^{\underline{o}}$ 2, 363-374.

[33] Y.I. Petunin and V.I. Savkin, Convergence generated by analytic functions, Ukranian. Math. Soc. 40 (1980), 676-679. 
[34] L.P. Rodrigues, Extensão de Polinômios para o Bidual de Certos Espaços de Banach e Tópicos Relacionados, Tese de Doutorado - IME-USP (2005).

[35] R.A. Ryan, Introduction to Tensor Products of Banach Spaces, Springer Monographs in Mathematics, Springer-Verlag, London, 2002.

[36] C.J. Seifert, The dual of Baernstein's space and the Banach-Saks property, Bull. Acad. Polon. Sci. Sér. Sci. Math. 26 (1978), no 3, 237-239.

[37] M.J. Sevilla and R. Payá, Norm attaining multilinear forms and polynomials on predual of Lorentz sequence spaces, Studia math. 127 (1998), nº 2, 99-112.

[38] C. Stegall, Dual of certain spaces with the Dunford-Pettis property, Notices Amer. Math. Soc. 19 (1972), $n^{\underline{o}}$ 7, 799.

[39] N.N. Tocha, As Propriedades P e RP em Espaços de Banach, Dissertação de Mestrado, IME-USP (2002).

[40] _ As Propriedades (P) e (RP) no predual do espaço de Stegall, Atas do $59^{\circ}$ Seminário Brasileiro de Análise, (2004), 170-176.

[41] __ Exemplos de funções em espaços de Banach com a propriedade ACL, Atas do 61ํo Seminário Brasileiro de Análise, (2005), 133-140.

[42] __ As propriedades (P) e (RP) nos espaços de Baernstein e de Lorentz, Atas do 62ํㅗ Seminário Brasileiro de Análise (CD-ROM) 31 (2005) no 2.

[43] B.S. Tsirelson, Not every Banach space contains an imbedding of $l_{p}$ or $c_{0}$. Funct. Anal. Appl. 8 (1974), no 2, 138-141. 